\title{
A REVIEW OF THE GENUS BLAPS (COLEOPTERA: TENEBRIONIDAE) OF CENTRAL AND SOUTH KAZAKHSTAN WITH DESCRIPTION OF TWO NEW SPECIES
}

\author{
Ivan Chigray ${ }^{1}$ and Alexander Ivanov ${ }^{2}$ \\ ${ }^{1}$ Zoological Institute of the Russian Academy of Sciences, Universitetskaya Emb. 1, Saint \\ Petersburg 199034, Russia; E-mail: chigray93@bk.ru, https://orcid.org/0000-0002-3830-6860 \\ ${ }^{2}$ Institute of Ecology of Plants and Animals Ural Branch of RAS, 8th March Str. 202, \\ Ekaterinburg 620144, Russia; E-mail: fluegel4@gmail.com \\ https://orcid.org/0000-0001-8500-8287
}

\begin{abstract}
A taxonomic review of the 32 species and subspecies of the genus Blaps Fabricius, 1775 from Central and South Kazakhstan is given. Two new species are described: Blaps skopini sp. n. and Blaps fouquei sp. n. The first species is most similar to Blaps granulata Gebler, 1825, B. granulipennis Skopin, 1966 and B. tsharynensis Skopin, 1961 but differs from these lastly mentioned species in the longer caudal extension of the elytra (mucro), shape and punctation of pronotum, structure of the male parameres and female genital tubes. Blaps fouquei sp. n. is most similar to B. seriata Fischer von Waldheim, 1820, and differs in the longer antennae, elytra flattened along suture, structure of the male parameres and female genital tubes. Blaps turcomanorum Seidlitz, 1893 is recorded for Kazakhstan for the first time. A key to all species known from the area under consideration is presented. Lectotypes of $B$. gigantea Motschulsky, 1845 and B. confusa Ménétriés, 1832 are designated. The most species are illustrated for the first time.
\end{abstract}

Key words: Blaps, Kazakhstan, taxonomy, distribution, new species.

\section{INTRODUCTION}

The largest genus in the tribe Blaptini Leach, 1815, Blaps Fabricius, 1775 includes more than 250 species, more than 30 of which are listed for Kazakhstan (LöвL et al. 2008). The most complete taxonomic revision of the genus Blaps (including Middle Asia and Kazakhstan) was published by SEIDLitz (1893), the disadvantages of his system we discussed earlier (CHIGRAY et al. 2016, Chigray \& Nabozhenko 2016).

Recently some progress in studies on the phylogeny and systematics of Blaps from the Western Mediterranean region (Soldati et al. 2009, Martínez Fernández 2010, Comdamine et al. 2011, Castro Tovar 2014, Kergoat et al. 2014, Soldati et al. 2017), the Caucasus (Abdurakhmanov \& Nabozhenko 2011) and China (REN et al. 2016) was reached. Species of Blaps from other regions, especially from Middle Asia, Kazakhstan, the Middle East, Afghanistan and Himalaya, need a further revision. Only a few taxonomic papers on these regions were published in the 21st century (Schawaller 2006, Chigray \& Nabo- 
zhenko 2016, Chigray et al. 2016, Nabozhenko et al. 2019). This paper aims to summarise new data on the fauna of the genus of Middle Asia and Kazakhstan.

Significant contributions to the knowledge of adults and larvae of Blaps of Kazakhstan were published by Sкорім (1960, 1961, 1964, 1966, 1968, 1973, 1977), who analysed faunistics, morphology and morpho-ecological evolution of Blaptini.

The taxonomic and faunistic reviews of Blaps of Western Kazakhstan, as well as some taxonomic problems were discussed in our previous work (Chigray et al. 2016). The genus is very diverse in Central and South Kazakhstan, but the status of some subspecies needs revision and molecular-genetic analysis. The fauna of the Blaps of Kazakhstan has been studied quite well, but discovery of new species continues (Medvedev 2004, Chigray et al. 2016). In this study two new species of Blaps from South Kazakhstan are described and a review of the genus Blaps from Central and South Kazakhstan is presented.

\section{MATERIAL AND METHODS}

The study is based on the examination of adult beetles from the following institutes, museums and private collections: ZIN - Zoological Institute of the Russian Academy of Sciences, St. Petersburg, Russia; IEPaAY - Institute of Ecology of Plants and Animals Ural Branch of the Russian Academy of Sciences, Yekaterinburg, Russia; NMP - Národní Museum, Prague, Czech Republic, NHM - Naturhistorisches Museum, Vienna, Austria. Scanning electron microscopy was made with the SEM EVO-40 XVP (LEO 143OVP) (Federal Research Centre the Southern Scientific Centre of the Russian Academy of Sciences, Rostov-on-Don, Russia).

South Kazakhstan includes the following provinces: Almaty, Jambyl, Turkistan, Kyzylorda; Central Kazakhstan includes only the Karaganda Region.

The system of MATthews and Bouchard (2008) is used for the abdomen: abdominal ventrites 1-5 (we use) or abdominal sternites III-VII.

The synonymy of the species was published in LöвL et al. (2008). The references include only the publications with records of Blaps in Kazakhstan and publications with original descriptions.

\section{TAXONOMY \\ Subgenus Blaps Fabricius, 1775}

Type species: Tenebrio mortisagus Linnaeus, 1758

Blaps ballioni Skopin, 1977

(Fig. 1)

Skopin, 1977: 151.

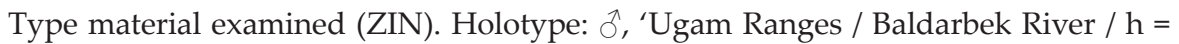
1800 m / 14.vi.1964 / leg. N. Skopin' [in Cyrillics]. Allotype: 1 +, 'Karzhantau Range / Badam River / h = 1600 m / 18.v.1938 / leg. N. Skopin' [in Cyrillics]. 
Distribution. Kazakhstan: Ugam and Karzhantau ranges (SкоріN 1977).

Blaps caraboides caraboides Allard, 1882

(Fig. 2)

Allard, 1882: 135; Seidlitz, 1893: 294; Skopin, 1960: 50 (larva); Skopin, 1961: 189.

Material examined (ZIN). 1 o, 'Southeast Kazakhstan / around Issyk Lake / vii. 1958 / leg. N.G. Skopin' [in Cyrillics]; 1 ऽ, 'Kazakhstan / southeast side of Ketmen Range / 20 km north of Sarynaz / $\mathrm{h}=2000$ / arid recess near crag / 3.vi.1986. / leg. L. Egorov' [in Cyrillics]; 1 †, ‘Kazakhstan / Alma-Ata / Big Almaty Lake / 21.vii.1958 / leg. N.G. Skopin’ [in Cyrillics].

Distribution. Kazakhstan, China (Gansu, Ningxia, Qinghai, Shaanxi, Xinjiang, Xizang provinces), Kyrgyzstan, Tajikistan, Afghanistan (Löвl et al. 2008).

Regional distribution. Southeast Kazakhstan and adjacent territories of Kyrgyzstan (Central Tian Shan) (Sкорім 1961).

\section{Blaps caraboides intermittens Kaszab, 1962}

(Fig. 3)

Kaszab, 1962: 313; Skopin, 1961: 189.
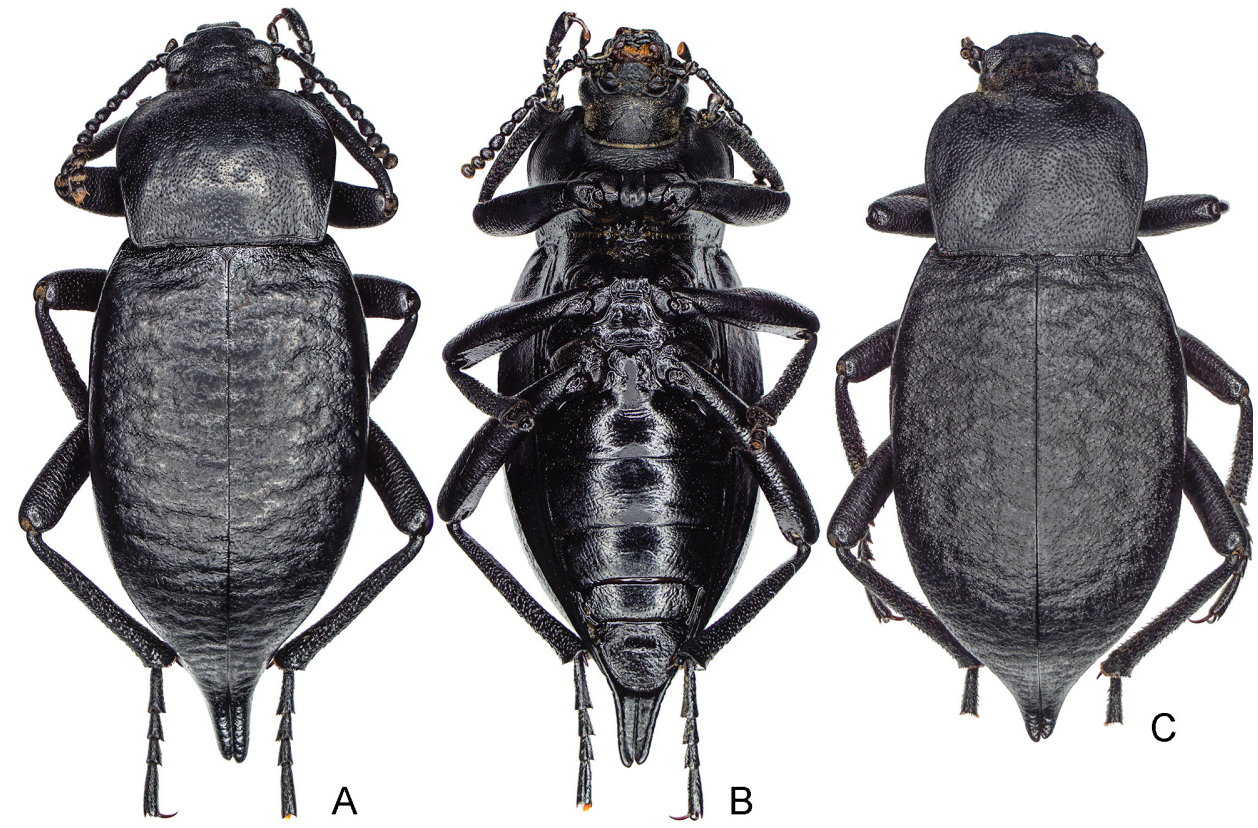

Fig. 1. B. ballioni, habitus. $\mathrm{A}, \mathrm{B}=\widehat{O} ; \mathrm{C}=\mathrm{q} ; \mathrm{A}, \mathrm{C}=$ dorsal view; $\mathrm{B}=$ ventral view 
Type material examined (ZIN). Paratypes: $6 \hat{\jmath} \hat{\jmath}$, ‘Southeast Kazakhstan / Dzungarian Alatau / Malyy Baskan River / 10.vi.1953 / leg. N. Balabas' [in Cyrillics].

Material examined (ZIN). 2 $え \widehat{\jmath}$, 'Southeast Kazakhstan / Dzungarian Alatau / Malyy Baskan River / 25 km east of Pokatilovka village / 18.vi.1968 / leg. G. Bugaev' [in Cyrillics] [45 $\left.23^{\prime} 22.90^{\prime \prime} \mathrm{N}, 80^{\circ} 8^{\prime} 25.18^{\prime \prime} \mathrm{E}\right]$.

Comments. The subspecies was formally described by Kaszab (1962), but a year before, SKopIN (1961) already indicated, without giving a name, that Kaszab considered the populations of the Dzhungarian Alatau as a separate subspecies.

Distribution. Kazakhstan: to the northeast of Ili River, spruce groves in Dzungarian Alatau (SKopin 1961, Kaszaв 1962).

\section{Blaps deplanata Ménétriés, 1832}

(Figs 4, 21A,B)

Ménétriés, 1832: 199; Fischer von Waldheim, 1832: 192 ("Blaps muricata"); Seidlitz, 1893: 288; Skopin, 1968: 86; Medvedev \& Nepesova, 1985: 119.

Type material examined (ZIN). Lectotype of Blaps deplanata (designated by AвDURAкhmanov \& Nabozhenкo 2011): đ̊, ‘Baku. // deplanata Menet. Baku // Lectotypus / Blaps
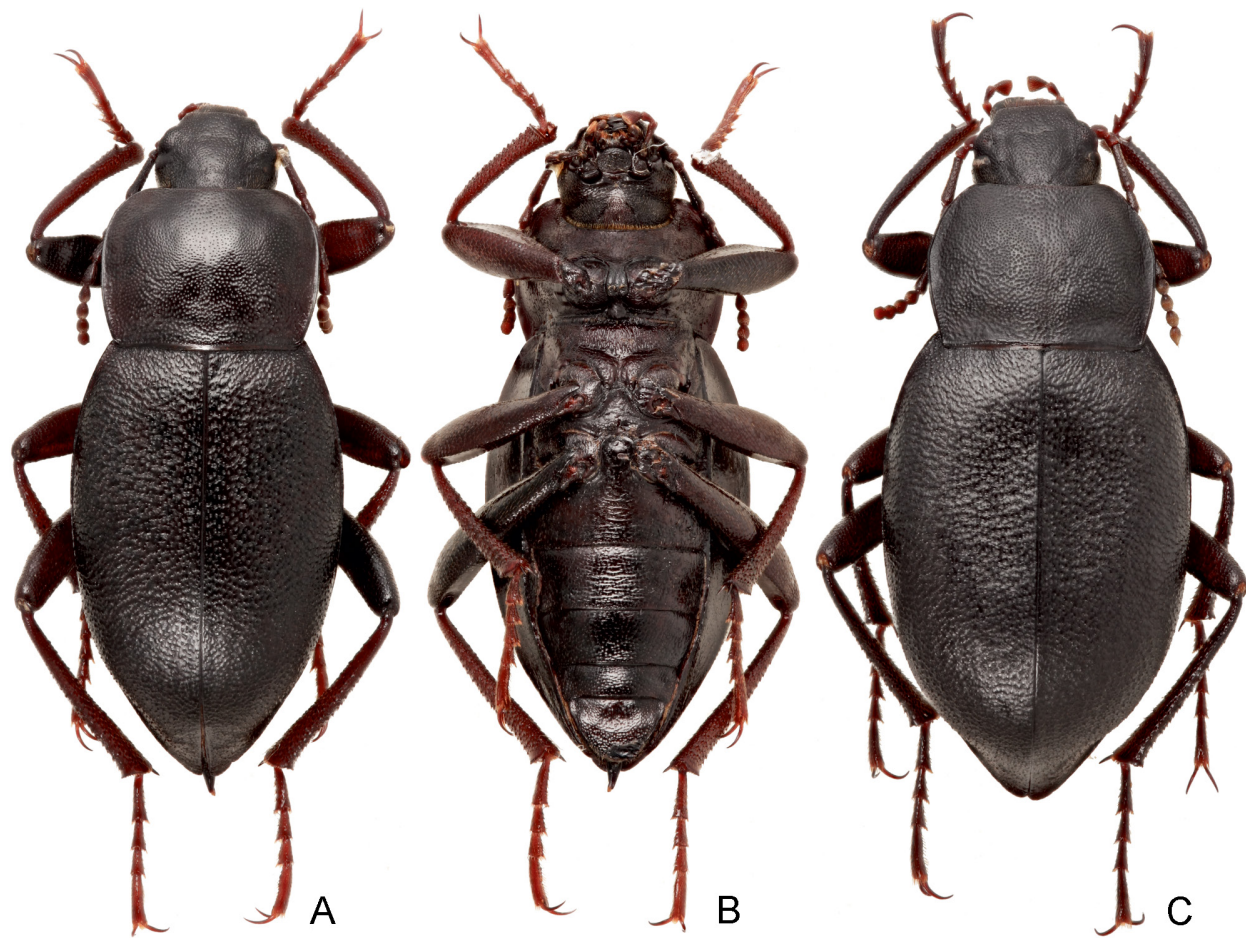

Fig. 2. $B$. caraboides caraboides, habitus. $A, B=\widehat{O} ; C=q ; A, C=$ dorsal view; $B=$ ventral view 
deplanata Mén. 1832 / des. Abdurakhmanov \& Nabozhenko'. Paralectotype: 1 \&, 'Baku. // Paralectotypus / Blaps deplanata Mén. 1832 / des. Abdurakhmanov \& Nabozhenko'. Lectotype of Blaps muricata (designated by AвDURAKHMANov \& NABOzHENKo 2011): $\widehat{\partial}$, 'Baku. // muricata Fisch. Baku // Lectotypus / Blaps deplanata Mén. 1832 / des. Abdurakhmanov \& Nabozhenko'.

Material examined (ZIN). 1 ㅇ, 'Kazakhstan / Barsa-Kelmes Island [former island of Aral Sea] / 13.xii.1978 / leg. A. Konev'; 1 ठ, ‘Uzbekistan / Between Bukhara and Kata Kurgan / v.[18]84. / leg. Regel'; 3 ổ, 2 우, ‘Uzbekistan / Juma - Samarkand / 12.vii.[18]98 / leg. Retter'; 1 ô, 'Uzbekistan / Around Samarkand / 1.iii.[18]96 / leg. L. Barshevsky' [in Cyrillics]; $11 \hat{\jmath} \widehat{\jmath}$, ‘Uzbekistan / Around Samarkand / 3.iii.[18]96 / leg. L. Barshevsky' [in Cyrillics]; 1 §’, 1 ㅇ, ‘Uzbekistan / Around Samarkand / 13-15.iv.[18]96 / leg. L. Barshevsky' [in Cyrillics]; 1 §’, ‘Uzbekistan / Samarkand / 1898 / leg. Retter'; 1 +, 'Uzbekistan / Samarkand district / Samarkand / 7.v.1904 / leg. G. Suvorov' [in Cyrillics]; 1 ð’, ‘Uzbekistan / Samarkand / 1892 / leg. O. Herz'; 1 đ’, ‘Uzbekistan / Samarkand / 1892 / leg. Herz // coll. Sivers'.

Distribution. Azerbaijan (Apsheron Peninsula), Iran, Kazakhstan, Turkmenistan, Uzbekistan (Abdurakhmanov \& Nabozhenko 2011, Medvedev \& NePEsova 1985).

Regional distribution. The Barsakelmes Nature Reserve (a former island in the Aral sea), the outskirts of Northwestern Tian Shan (the Boroldaytau mountains) (SKоргN 1968).
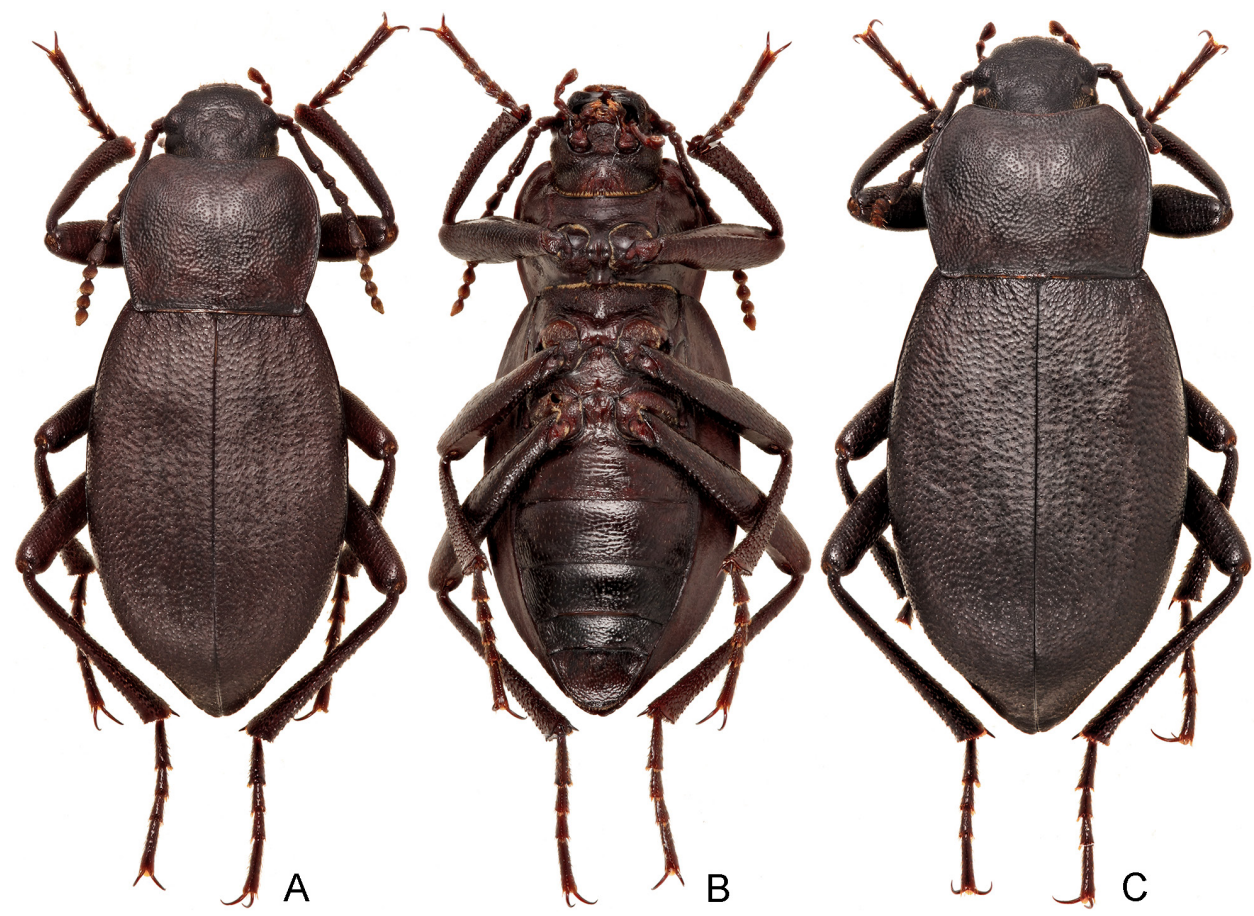

Fig. 3. B. caraboides intermittens, habitus. $A, B=C ; C=+; A, C=$ dorsal view; $B=$ ventral view 

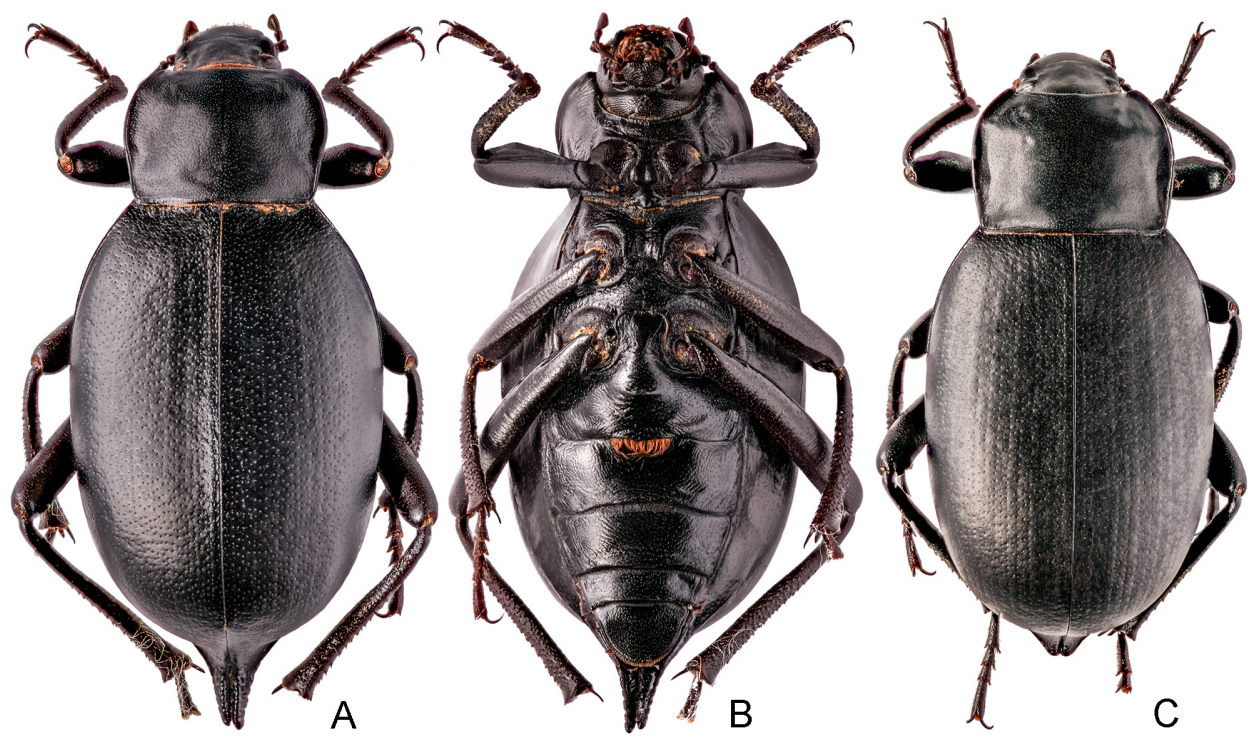

Fig. 4. $B$. deplanata, habitus. $\mathrm{A}, \mathrm{B}=\hat{\mathrm{O}} ; \mathrm{C}=\uparrow ; \mathrm{A}, \mathrm{C}=$ dorsal view; $\mathrm{B}=$ ventral view
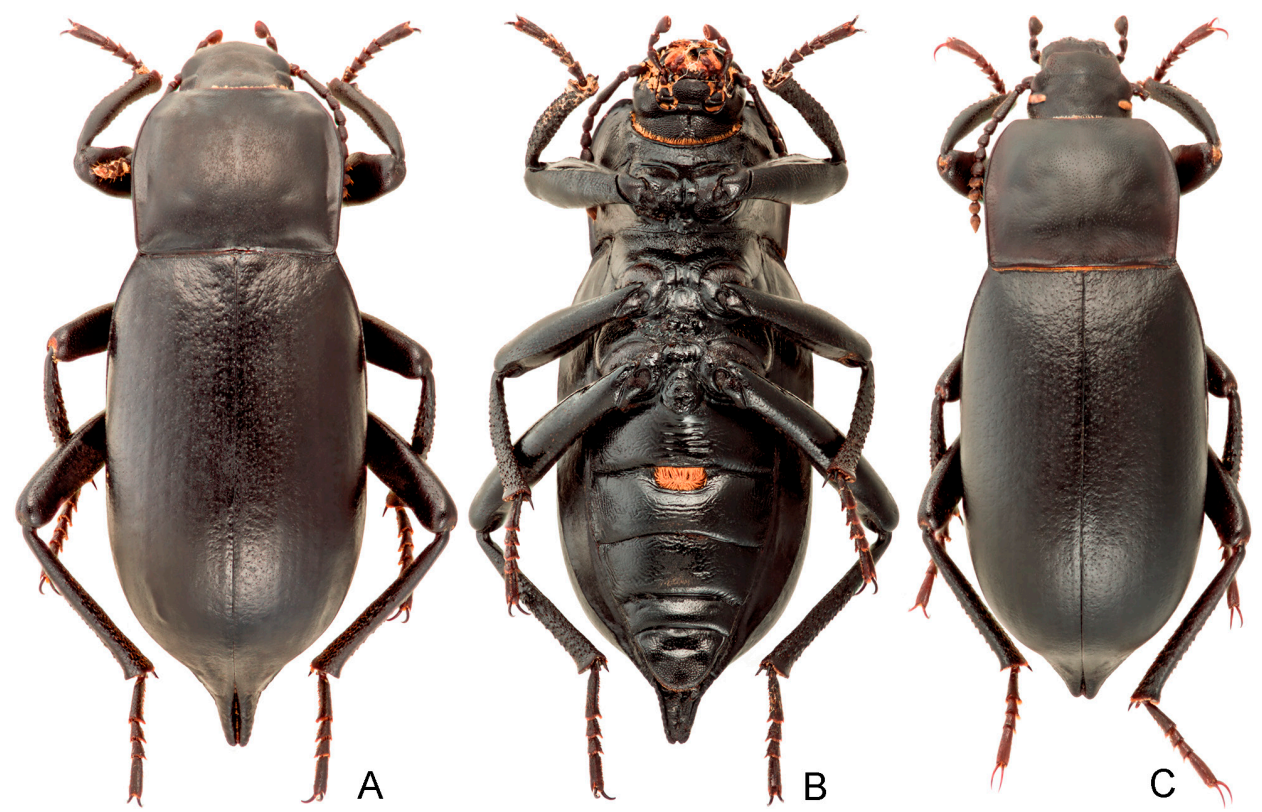

Fig. 5. B. evanida, habitus. $\mathrm{A}, \mathrm{B}=\hat{\mathrm{O}} ; \mathrm{C}=\mathrm{O} ; \mathrm{A}, \mathrm{C}=$ dorsal view; $\mathrm{B}=$ ventral view 
Blaps evanida Seidlitz, 1893

(Figs 5, 27D)

Seidlitz, 1893: 285; Skopin, 1960: 55 (larva); Skopin, 1961: 191; Skopin, 1968: 86.

Material examined (ZIN). 1 +, ‘Southeast Kazakhstan / Alma-Ata Region / Kurdayskaya station / h = 900 m / 07.viii.1907 / leg. A. Jakobson' [in Cyrillics]; 2 ô 0,8 우, 'Southeast Kazakhstan / Alma-Ata Region / Kurdayskiy pass / h = 1100 m / 16.v.1907 / leg. A.

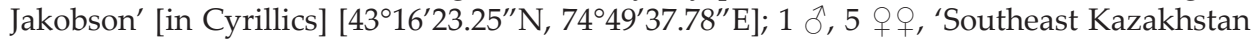
/ Alma-Ata Region / Vernyi (Alma-Ata) suburbs / h = 900 m / 23-29.v.1907 / leg. A. Jakob-

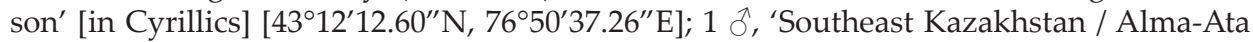
Region / Kara-Bulak village / 24.vii.1909 / leg. I.E. Boikov' [in Cyrillics].

Distribution. Kazakhstan (Löвl et al. 2008), “Turkestan” (Sкоріn 1961).

Regional distribution. The plain near Trans-Ili Alatau Range (SкоріN 1960), northern slopes and foothills of Trans-Ili Alatau Range (SкоріN 1961), Zhetizhol Range, southeastern part of Karatau Ridge (SKopIN 1968).

\section{Blaps faustii Seidlitz, 1893}

(Figs 6, 27E)

Seidlitz, 1893: 305; Skopin, 1968: 86.

Material examined (ZIN). 1 đ , 'Kazakhstan / Around Kazalinsk / Dorandsh / 20.x.[18]75 // 79221' [in Cyrillics]; 1 ơ: 'Uzbekistan / Bukhara / Amu Darya / Kelif / 10.vi.1904 / leg. Suvorov' [in Cyrillics]; 1 ㅇ, ‘Uzbekistan / Bishkent / Bukhara / 8.v.[18]97 / leg. Kazankov' [in Cyrillics].
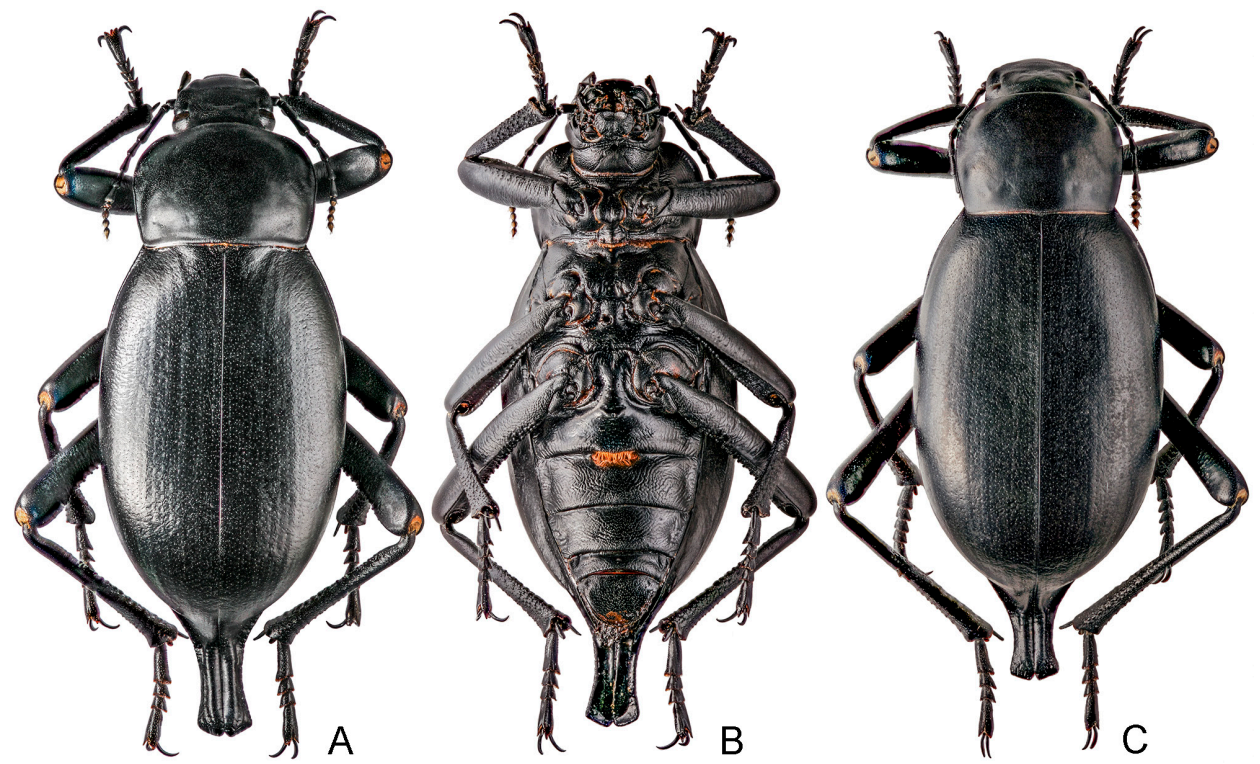

Fig. 6. B. faustii, habitus. $\mathrm{A}, \mathrm{B}=\hat{\mathrm{O}} ; \mathrm{C}=\mathrm{O} ; \mathrm{A}, \mathrm{C}=$ dorsal view; $\mathrm{B}=$ ventral view 
Distribution. Kazakhstan, Uzbekistan, Turkmenistan, Tajikistan, Afghanistan (LöBL et al. 2008).

Regional distribution. Northern and northeastern parts of the Kyzylkum desert (SKOPIN 1968).

\section{Blaps fouquei sp. n.}

(Figs 7-11)

Type material (ZIN). Holotype: $\lesssim$, 'South Kazakhstan / Akmechet-Aulie Cave / 100 km NW Bourny / 22.vi.1964 / leg. V. Kusov // coll. N. Skopin'. Paratypes: 1 + ,'South Kazakh-

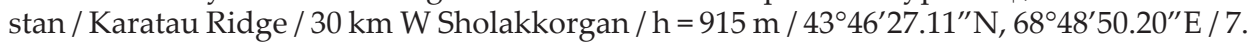
vi.2015 / leg. A. Shapovalov'; 1 đ: 'South Kazakhstan / Karatau Ridge / 15 km S Kozmoldak

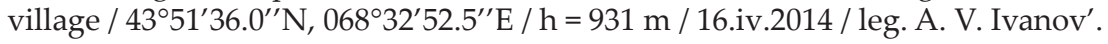

Description. Male. Body black, mat, slender. Anterior margin of epistoma weakly arcuately emarginate, straight in middle. Lateral margins of epistoma weakly rounded. Lateral margins of genae straight in anterior half, rounded at base. Lateral margins of head with distinct emargination between epistoma and genae. Head widest at level of poste-
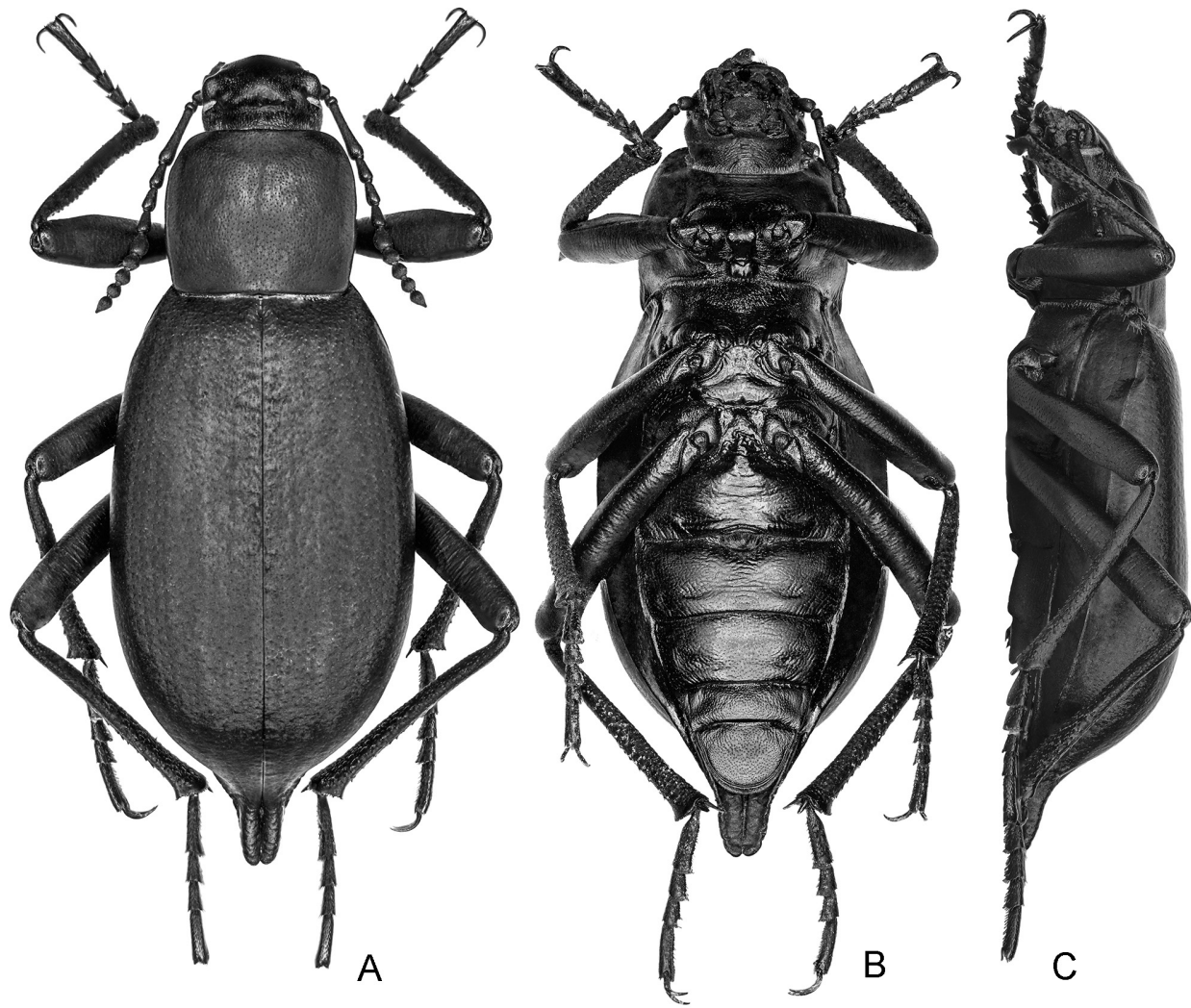

Fig. 7. B. fouquei sp. n., habitus, $\hat{\jmath}$. $A=$ dorsal view; $B=$ ventral view; $C=$ lateral view 
rior margin of eyes. Head 1.45 times as wide as interocular distance. Antennomeres 10-11 reaching base of elytra. Ratio of length/width of antennomeres 2-11 as 7(9), 43(10), 19(10), 17(10), 17(11), 18(14), 10(9), 10(9), 10(9), 12(10). Mentum transversely oval, convex in middle, its base straight. Punctation of head fine, sparse (puncture diameters on frons 5 times as wide as distance between punctures, puncture diameters on genae 2 times as wide as distance between punctures).

Pronotum weakly transverse (1.15 times as wide as long), widest at middle, 1.72 times as wide as head. Ratio of pronotal width near anterior angles to widest part and width at base $5.3: 7.7: 7.2$. Anterior margin of pronotum widely emarginate, lateral margins widely rounded, base straight. Lateral margins of pronotum weakly emarginate near base. Disc of pronotum weakly convex, lateral sides narrow flattened. Anterior angles obtuse, widely rounded, posterior angles right, narrowly rounded. Pronotum completely beaded, except for middle of apex and base. Pronotal punctation moderately coarse, dense (distance between punctures in middle subequal to one puncture diameter), punctation of lateral sides and near base sparser (puncture diameters 2 times as wide as distance between punctures). Prothoracic hypomera with small wrinkles and covered with small granules. Lateral margins of hypomera not excavate.

Elytra weakly convex, elongate (1.93 times as long as wide together), flattened along suture, widest at middle, 3.42 times as long and 2.52 times as wide as pronotum, 2.52 times as wide as head. Caudal extension of elytra (mucro) distinct, $2.2 \mathrm{~mm}$ long; elytra 10 times as long as mucro. Elytral surface with fine microwrinkles and rasp-like punctation, obliterated from base to middle, and almost completely disappearing closer to elytral apex. Epipleura with fine wrinkles and sparse fine rasp-like punctures. Hair tuft between
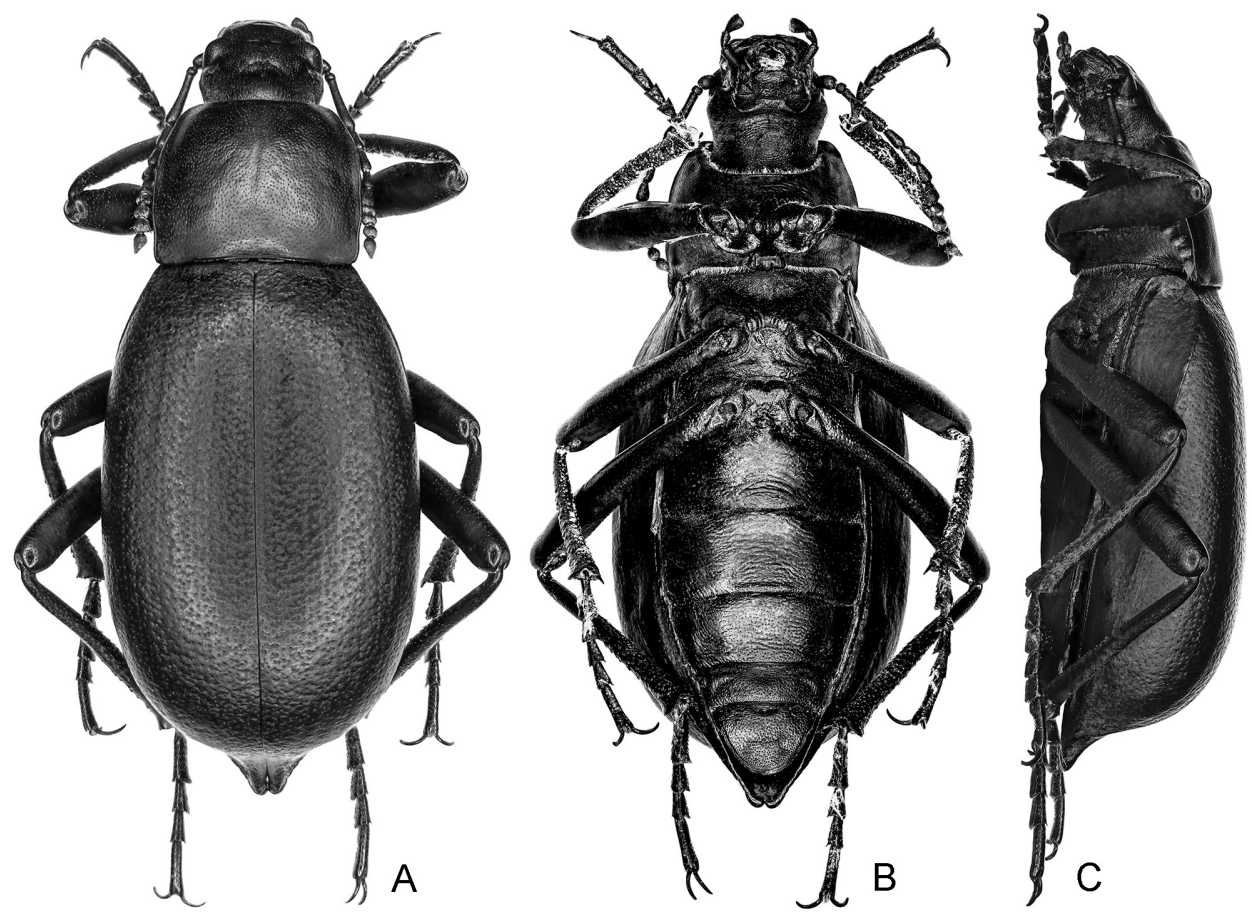

Fig. 8. B. fouquei sp. n., habitus, + . $A=$ dorsal view; $B=$ ventral view. $C=$ lateral view 
abdominal ventrites 1 and 2 present. Abdominal ventrite 1 without tubercle. Abdominal ventrites 1-3 with sparse rasp-like punctation and sparse granules, covered with short recumbent hairs. Abdominal ventrites $4-5$ with moderately dense punctation, punctation at lateral sides of ventrite 4 forming wrinkles, ventrite 5 completely beaded, except for base and with unclear bead apically.

Anterior margin of male inner sternite VIII emarginate; accessory gland of sternite VIII moderately long and thin. Rods of spiculum gastrale not merged at apex, forming long common stem. Aedeagus length $4.5 \mathrm{~mm}$, width $0.9 \mathrm{~mm}$. Aedeagus weakly C-curved. Lateral margins of parameres weakly rounded, widely emarginate in apical third, lateral sides of parameres with longitudinal impression in basal two-thirds, apex rounded. Parameres length $1.3 \mathrm{~mm}$, width $0.9 \mathrm{~mm}$.

Legs long, slender. Ratio of lengths of femora, tibiae and tarsi of fore, middle and hind legs $8.4: 7.3: 5.3,9.4: 7.9: 6.1,11.6: 10.5: 7.8$. All tarsomeres with one pair of setal brushes.
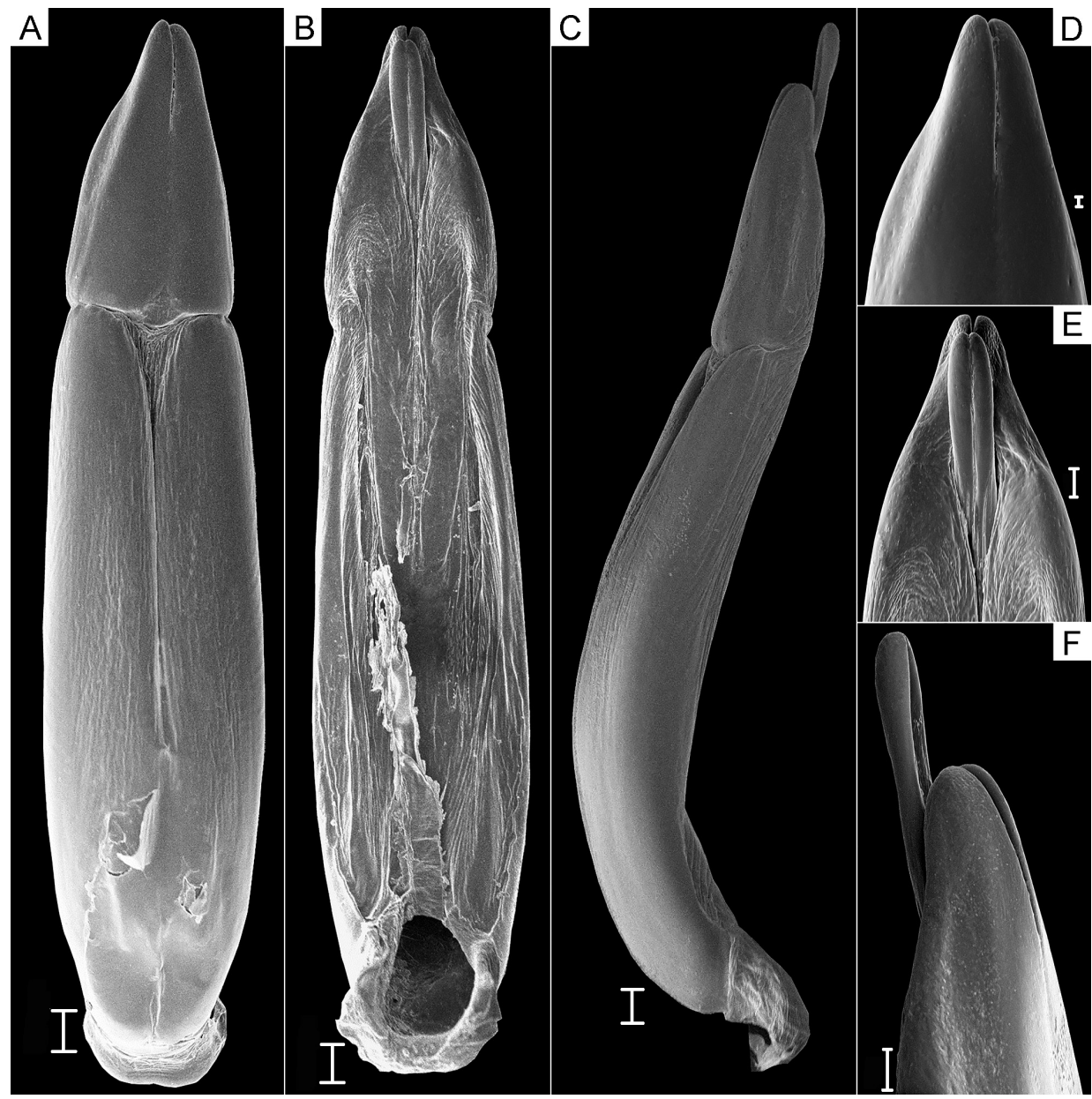

Fig. 9. $B$. fouquei sp. $\mathrm{n}$., aedeagus. $\mathrm{A}=$ dorsal view; $\mathrm{B}=$ ventral view; $\mathrm{C}=$ lateral view; $\mathrm{D}=$ apical piece, dorsal view; $\mathrm{E}=$ the same, ventral view; $\mathrm{F}=$ the same, lateral view. Scale bars: $100 \mu \mathrm{m}$ for $\mathrm{A}-\mathrm{C}, 30 \mu \mathrm{m}$ for $\mathrm{D}, 200 \mu \mathrm{m}$ for $\mathrm{E}-\mathrm{F}$ 
Body length 29-30 mm, width 11-11.1 mm.

Female. Shape of body and punctation similar to those of male. Head 1.41 times as wide as interocular distance. Antennomere 11 reaching base of pronotum. Ratio of pronotal width near anterior angles, in widest part and at base $4.9: 7.0: 6.9$. Elytra elongate (1.76 times as long as wide together), 3.65 times as long and 1.55 times as wide as pronotum, 2.4 times as wide as head. Mucro short (1 mm).

Ovipositor moderately long. Apical lobes straight in basal two-thirds, weakly arcuately emarginate at apical third. Apex of lobes acute. Ventral side of lobes with excavation near middle. Middle of ventral side of lobes with small deep longitudinal wrinkles. Ante-

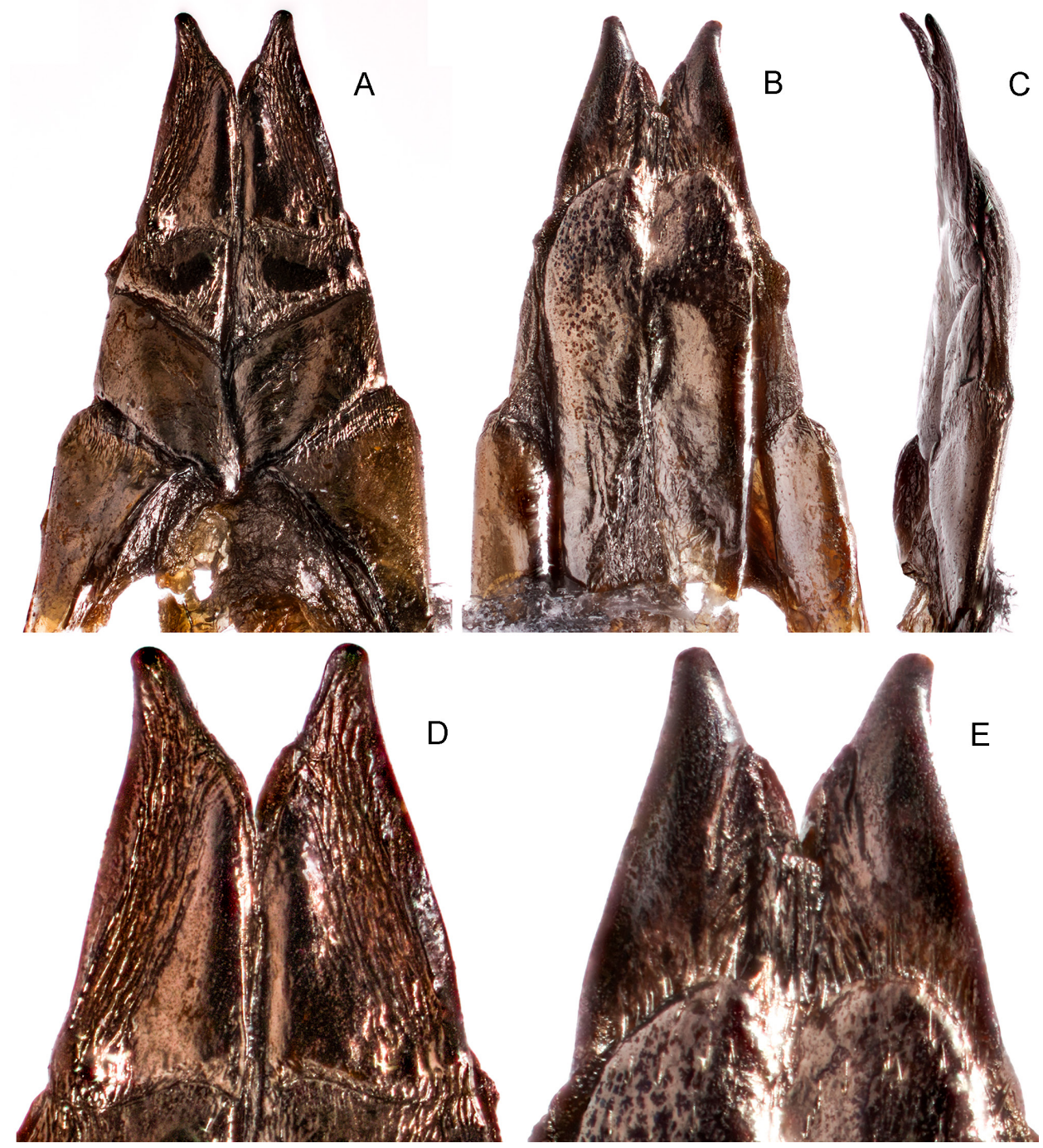

Fig. 10. B. fouquei sp. $n . A-C=$ ovipositor: $A=$ ventral view; $B=$ dorsal view; $C=$ lateral view; $\mathrm{D}-\mathrm{E}=$ apical lobes: $\mathrm{D}=$ ventral view $\mathrm{E}=$ dorsal view 


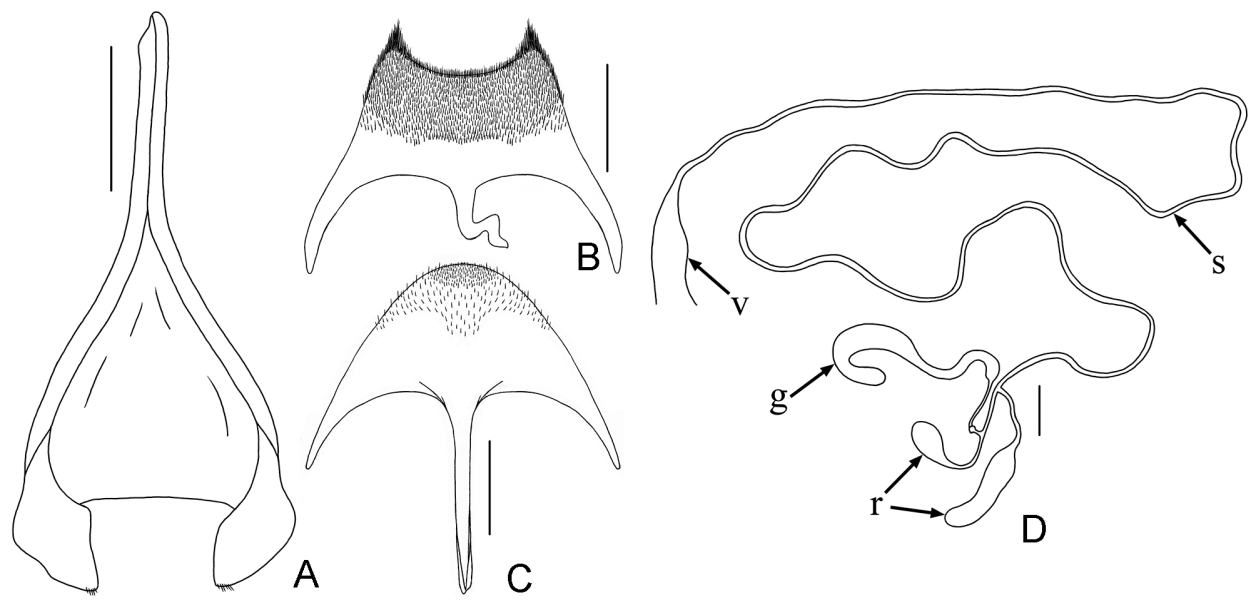

Fig. 11. B. fouquei sp. n., details of structure. $\mathrm{A}=$ spiculum gastrale; $\mathrm{B}=$ male inner sternite VIII, $\mathrm{C}=$ spiculum ventrale $; \mathrm{D}=$ female genital tube $(\mathrm{v}=$ vagina, $\mathrm{s}=$ basal duct of spermathe$\mathrm{ca}, \mathrm{r}=$ reservoirs, $\mathrm{g}=$ accessory gland of spermatheca). Scale bars $=1 \mathrm{~mm}$
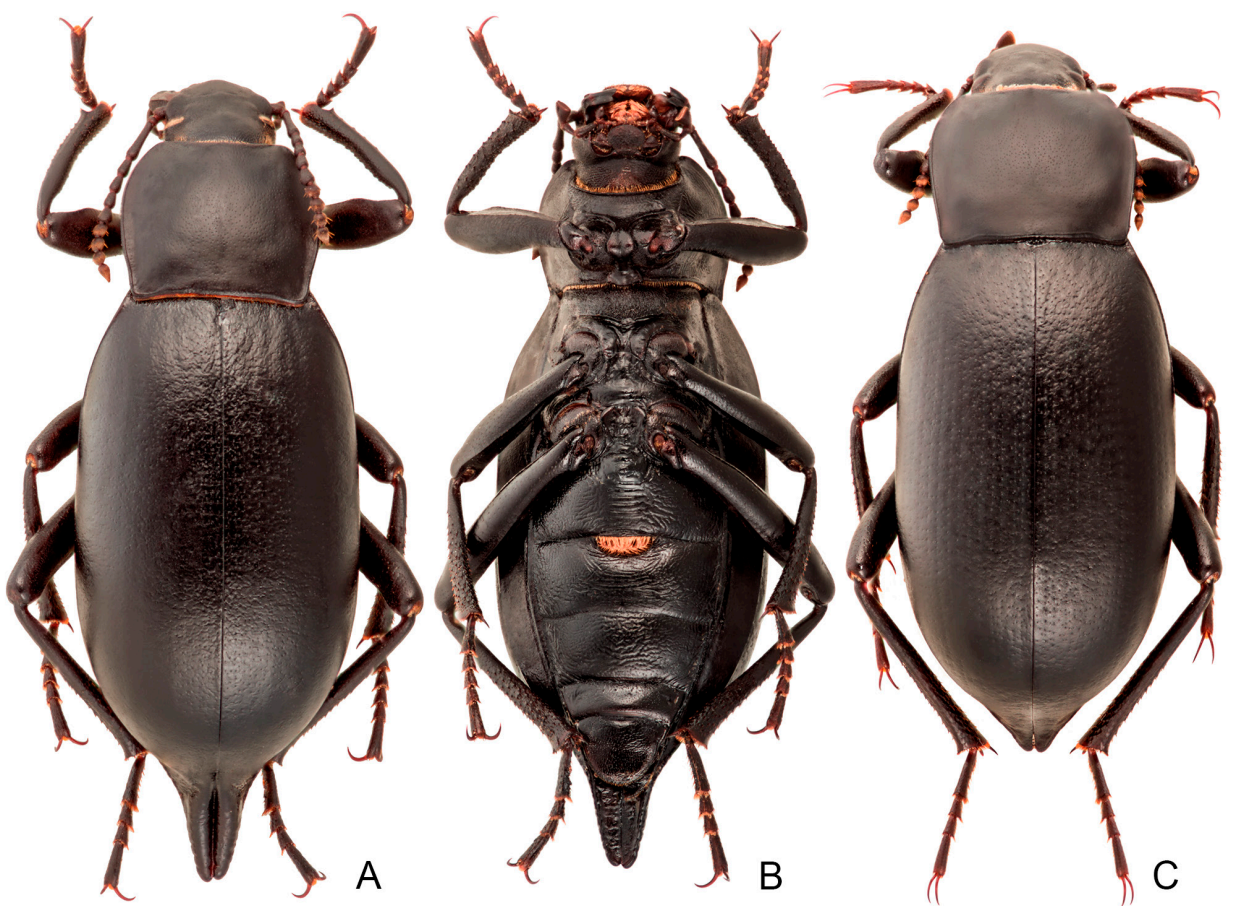

Fig. 12. $B$. seriata, habitus. $A, B=\hat{O} ; C=q ; A, C=$ dorsal view; $B=$ ventral view 
rior margin of proctiger widely emarginate. Basal duct of spermatheca between vagina and reservoirs long, gland of spermatheca short. Reservoirs of spermatheca separated from each other; 1st reservoir sharply expanding from middle, wide in apical half; 2nd reservoir almost spherical at apex, 1st reservoir slightly larger than 2nd. Base of spermathecal gland very thin. Stem of spiculum ventrale moderately long and wide.

Body length $27.3 \mathrm{~mm}$, width $11.1 \mathrm{~mm}$.

Etymology. The species epithet of this new species is dedicated to the late Czech entomologist, René Fouque.

Differential diagnosis. The body shape of the new species is similar to that in Blaps seriata Fischer von Waldheim, 1820 (Fig. 12), and differs from the latter in the following character states: antennomeres 10-11 of Blaps fouquei sp. n. reaching first quarter of elytra, those of $B$. seriata reaching only basal quarter of the pronotum; elytra of Blaps fouquei sp. $\mathbf{n}$. flattened along the suture, elytra of B. seriata weakly convex; lateral sides of parameres of Blaps fouquei sp. n. weakly rounded in the basal two-thirds, apical third widely emarginate, lateral sides of parameres of $B$. seriata weakly rounded; reservoirs of the spermatheca in Blaps fouquei sp. n. are separated from each other, reservoirs of the spermatheca and valve of the accessory gland in B. seriata are closely located.

\section{Blaps granulata Gebler, 1825}

SKopIN $(1966,1968)$ presented keys to adults for subspecies of Blaps granulata. Multiple subspecies (seven described, two scheduled for description) of B. granulata raises doubts. The validity of these taxa requires careful analysis using molecular-genetic methods; keys of Skopin, based on the elytral granulation, is unclear and difficult to use in identification of the taxa. In this paper, we present material on four Kazakhstanian subspecies of B. granulata (material on B. granulata stackelbergi Bogatchev, 1952 was not studied), but only the nominotypical subspecies is included in our key.

\section{Blaps granulata altynemelis Skopin, 1966}

(Fig. 13)

Skopin, 1966: 337; Skopin, 1968: 85.

Type material examined (ZIN). Holotype: $\hat{0}$, 'Southeast Kazakhstan / Dzungarian Alatau / Altyn-Emel Range / 29.viii.1962 / leg. N.G. Skopin' [in Cyrillics]. Allotype: 1 , 'Southeast Kazakhstan / upper terrace of Ili River / 24.viii.1962 / leg. N.G. Skopin' [in Cyrillics]. Paratypes: 1 o , 1 어, 'Southeast Kazakhstan / Dzungarian Alatau / Altyn-Emel Range / 30.viii.1962 / leg. N.G. Skopin' [in Cyrillics]; 1 + , 'Southeast Kazakhstan / Alma-Ata Region / 
Sary-Ozek suburbs / 04.vii.1962 / leg. N.G. Skopin' [in Cyrillics] [44²1'7.37"N, 7759'5.12"E]; 1 , 'Southeast Kazakhstan / Sarkand / 10.iv.1965. / leg. N. Yerolskaya' [in Cyrillics].

Material examined (ZIN). 2 우어, 'Southeast Kazakhstan / northern slope of AltynEmel Range / Kugaly suburbs / 16.ix.1966 / leg. N.G. Skopin' [in Cyrillics] [44²5'56.56”N, $78^{\circ} 42^{\prime} 26.28^{\prime \prime}$ E]; 1 ㅇ, 'Semirechye Region / env. of Araltobe / 14.ix.1966 / leg. N. Skopin' [in Cyrillics]; 10 ,' 'Southeast Kazakhstan / env. of Tekeli / 22.v.1967 / leg. V. Linsky' [in Cyrillics].

Distribution. Kazakhstan (Almaty Region) (LöвL et al. 2008).

Regional distribution. Altyn-Emel Range (SKopIN 1966, 1968).

\section{Blaps granulata granulata Gebler, 1825}

(Figs 14, 27B)

Gebler, 1825: 47; Seidlitz, 1893: 287; Skopin, 1961: 189: Skopin, 1966: 336; Skopin, 1968: 85; Arnoldi \& Medvedev, 1969: 403; Ren et al., 2016: 119.

Material examined (ZIN). 1 o, 'Southeast Kazakhstan / Alma-Ata Region / bottom land of Bolshaya Almatinka River / h = 1000 m / 02.vi.1949 / leg. N.G. Skopin' [in Cyrillics]; $1 \hat{\delta}$, 'Southeast Kazakhstan / Alma-Ata Region / Ketmen Range / Podgornoye suburbs /
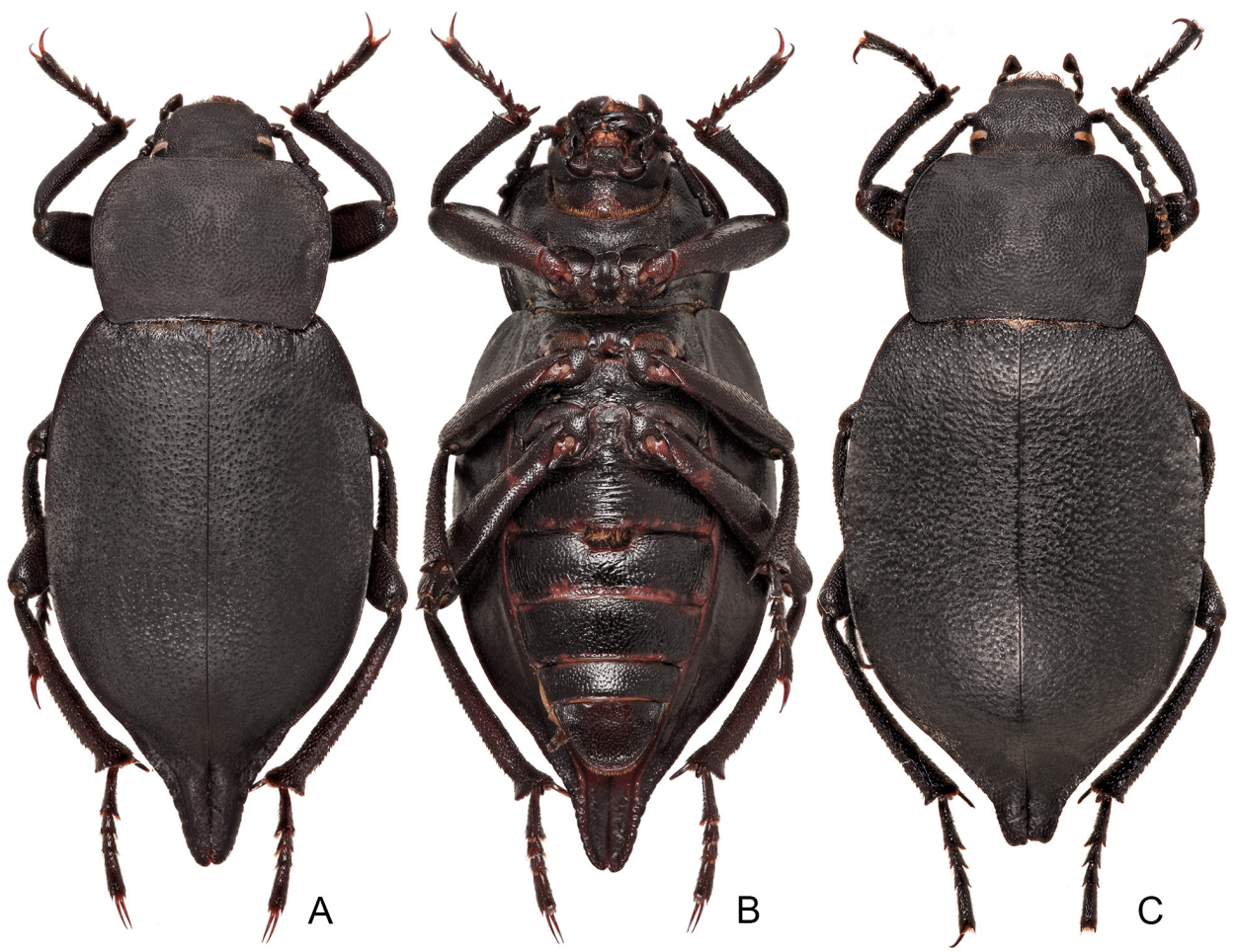

Fig. 13. B. granulata altynemelis, habitus. $A, B=\widehat{O} ; C=\rho ; A, C=$ dorsal view; $B=$ ventral view 


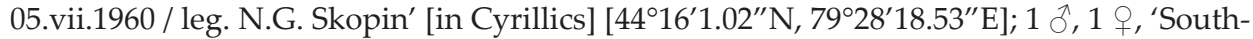
east Kazakhstan / South-Western Balkhash Lake region / Chu-Iliyskiye gory / 28.vii.1965 / leg. V. Kombulin' [in Cyrillics]; 1 o, 'Southeast Kazakhstan / Moyunkum Sands / Kos-

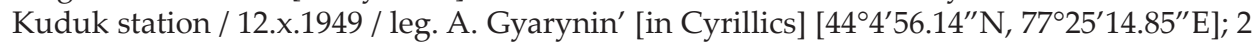
$\hat{\delta} \widehat{\partial}$, 'Southeast Kazakhstan / Alma-Ata Region / Kurtogay natural boundary / 21.vii.1959 /

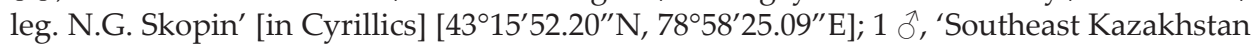
/ Sands along Khorgos River / 17.iv.1965 / leg. N.G. Skopin' [in Cyrillics]; 1 đ, 'Southeast Kazakhstan / Alma-Ata Region / Alma-Ata / Zailiyskiy Alatau Range / 16.v.1964

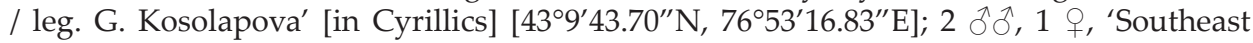
Kazakhstan / Alma-Ata Region / Tekeli suburbs / 22.v.1967 / leg. V. Linskiy' [in Cyril-

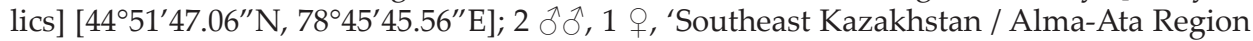
/ Dzungarian Alatau / Koktuma village / 26.vi.1962 / leg. G.S. Medvedev' [in Cyrillics] [45 51'17.07"N, 8138'20.27"E]; 1 + , 'Southeast Kazakhstan / Alma-Ata Region / Karatal River / 16.vi.1964 / leg. N. Gorbunov' [in Cyrillics]; 1 ô', 'Southeast Kazakhstan / AlmaAta Region / lower reaches of Lepsy River / 16.vi.1965 / leg. N.G. Skopin' [in Cyrillics]; 1 ô, 'Southeast Kazakhstan / Alma-Ata Region / Alma-Ata / 03.vi.1957 / leg. N.G. Skopin'

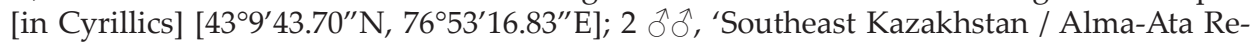
gion / Kokishbay village / 16.ix.1948 / leg. D. Aleksandrov' [in Cyrillics] [45²'19.10”N, 75²6'55.43”'E]; 1 §’, 'Southeast Kazakhstan / Alma-Ata Region / Akkol village / 13.vii.1969 /

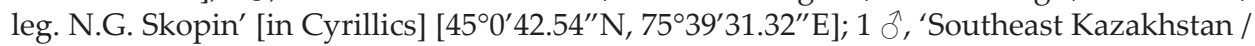
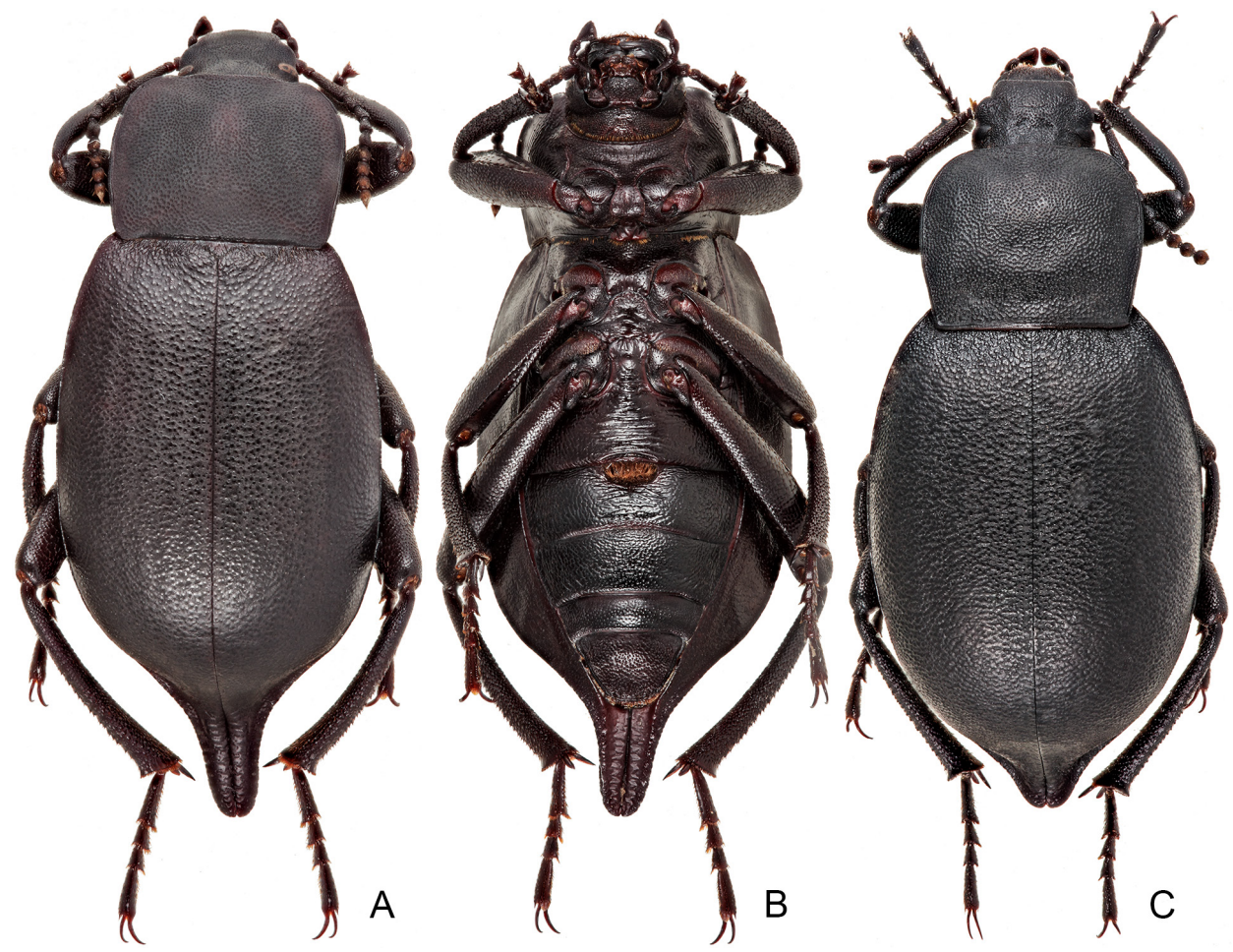

Fig. 14. B. granulata granulata, habitus. $A, B=\widehat{C} ; C=q ; A, C=$ dorsal view; $B=$ ventral view 
Alma-Ata Region / Dzungarian Alatau / 30 km south-west of Sarkand city / 15.vi.1968 / leg. T. Bugaev' [in Cyrillics] [45 $\left.18^{\prime} 41.75^{\prime \prime} \mathrm{N}, 79^{\circ} 33^{\prime} 25.99^{\prime \prime} \mathrm{E}\right] ; 10$, 'Southeast Kazakhstan / AlmaAta Region / Araltobe suburbs / 14.09.1966 / leg. N.G. Skopin' [in Cyrillics] [45²'10.45”N, 75²7'11.49"E]; 1 ô, 1 ㅇ, 'Southeast Kazakhstan / Alma-Ata Region / 40 km south of Khorgos village / 14.iv.1965 / leg. N.G. Skopin' [in Cyrillics] [4352'13.06"N, 80¹4'24.21"E]; 1 $\delta$, 'Southeast Kazakhstan / Jambyl Region / 120 km west of Burubayltal village / 15.ix.1964

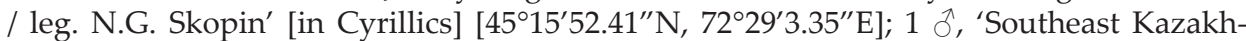
stan / Alma-Ata Region / Bakanas village / 09.vi.1953 / leg. A. Savicheva' [in Cyrillics]

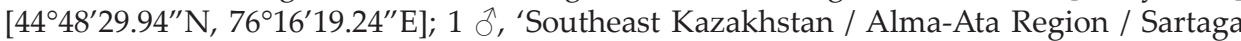
[env. of Narynkol village] / 24.iv.1964 / leg. N.G. Skopin' [in Cyrillics] [4243'12.29"N, 80 9'57.62”E]; 7 ふふふ, 3 우, ‘Eastern Kazakhstan / Saykan Range / Kendyrlik (Saryterek) village

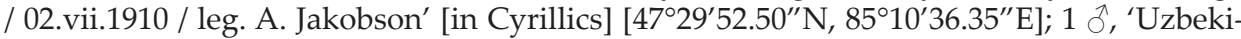
stan / Malyy Chimgan Mount / 23.vi.1936 / leg. N.G. Skopin’ [in Cyrillics] [41³2'45.11”N, $70^{\circ} 2^{\prime} 8.62$ 'E]; 1 o', 'Kyrgyzstan / Kyrgyz Ridge / Shamsi ravine / viii.1958 / leg. Pivovarov' [in Cyrillics] [42 $\left.39^{\prime} 17^{\prime \prime} \mathrm{N}, 75^{\circ} 23^{\prime} 20^{\prime \prime} \mathrm{E}\right]$.

Comments. Specimens labelled as "Blaps granulata mixta" and "Blaps granulata psammophila" are deposited in ZIN and NMP collections (prepared by Skopin for description, but remained undescribed). The specimens have no
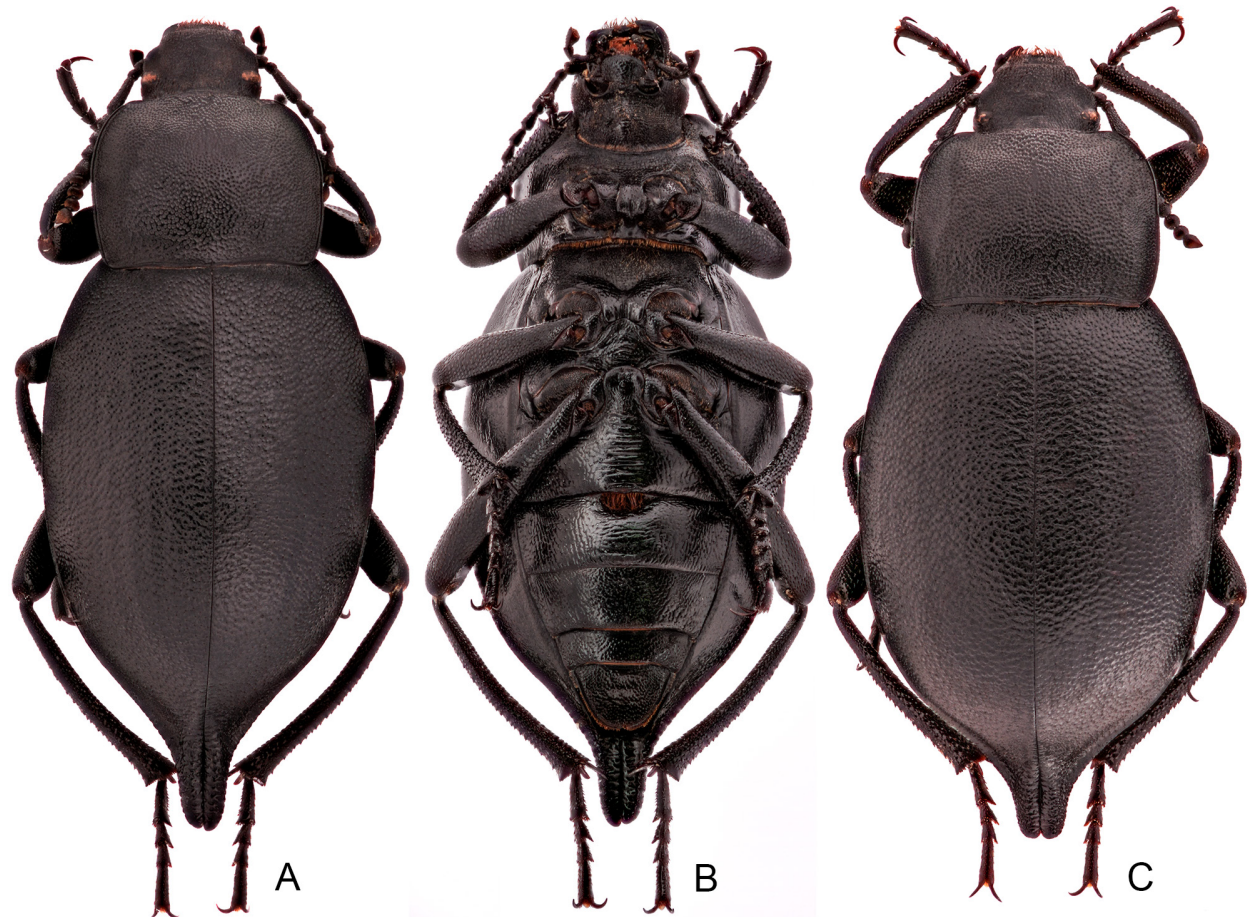

Fig. 15. B. granulata mixta, habitus. $A, B={ }^{\top} ; C=q ; A, C=$ dorsal view; $B=$ ventral view 
distinct differences from the nominotypical subspecies and interpreted here as Blaps granulata granulata.

Additional material. "Blaps granulata mixta" (unpublished name) (Figs 15A-C). Material examined (ZIN). $3 \hat{\sigma} \widehat{\delta}, 3$ 우, 'Southeast Kazakhstan / Alma-Ata Region / Jabyrtau

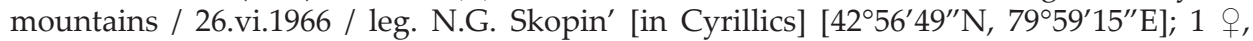
'Southeast Kazakhstan / Alma-Ata Region / Narynkol village / 30.v.1961 / leg. V. Chekalin'

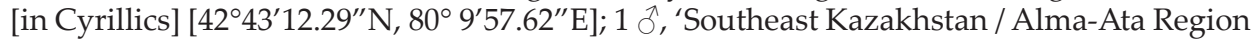
/ Terskey Alatau Range / Koksay River / 08.vi.1957 / leg. L. Marinenko' [in Cyrillics]; 1 ㅇ, 'Southeast Kazakhstan / Alma-Ata Region / Ketmen Range / 20.iii.1960 / leg. V. Zadorin'

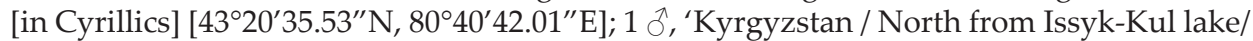
Chong-Örüktü village / 2.v.1956 / leg. N. Skopin' [in Cyrillics].

"Blaps granulata psammophila" (unpublished name) (Figs 16A-C). Material examined. 1 d, 'Thian-S. Musart' (NMP); 1 + (NMP), 'Chan-Tengri. Mont. merid.' (NMP); 1 + , 'Turkestan, Reitter leg.' (NMP); 1 đ̊, 'Southeast Kazakhstan / Panfilov [Zharkent city] / 12.05.1960 / leg. N. Skopin' [in Cyrillics] (ZIN); $1 \hat{\delta}$, 'caudata Gebl. Turcom.' (NMP); 1 q, 'Southeast Kazakhstan / Panfilov [Zharkent city] / 12.05.1960 / leg. N. Skopin' [in Cyrillics] (ZIN).

Distribution. Central and Southeast Kazakhstan and adjacent areas of Kyrgyzstan (Skopin 1960, 1961; Arnoldi \& Medvedev 1969), China (Gansu and Xinjiang provinces) (Löвl et al. 2008, Ren et al. 2016).

Regional distribution. Eastern part of Kyrgyz Range, the mountainous part of Talas River area, eastern part of Trans-Ili Alatau Range (Sкоргм 1968).
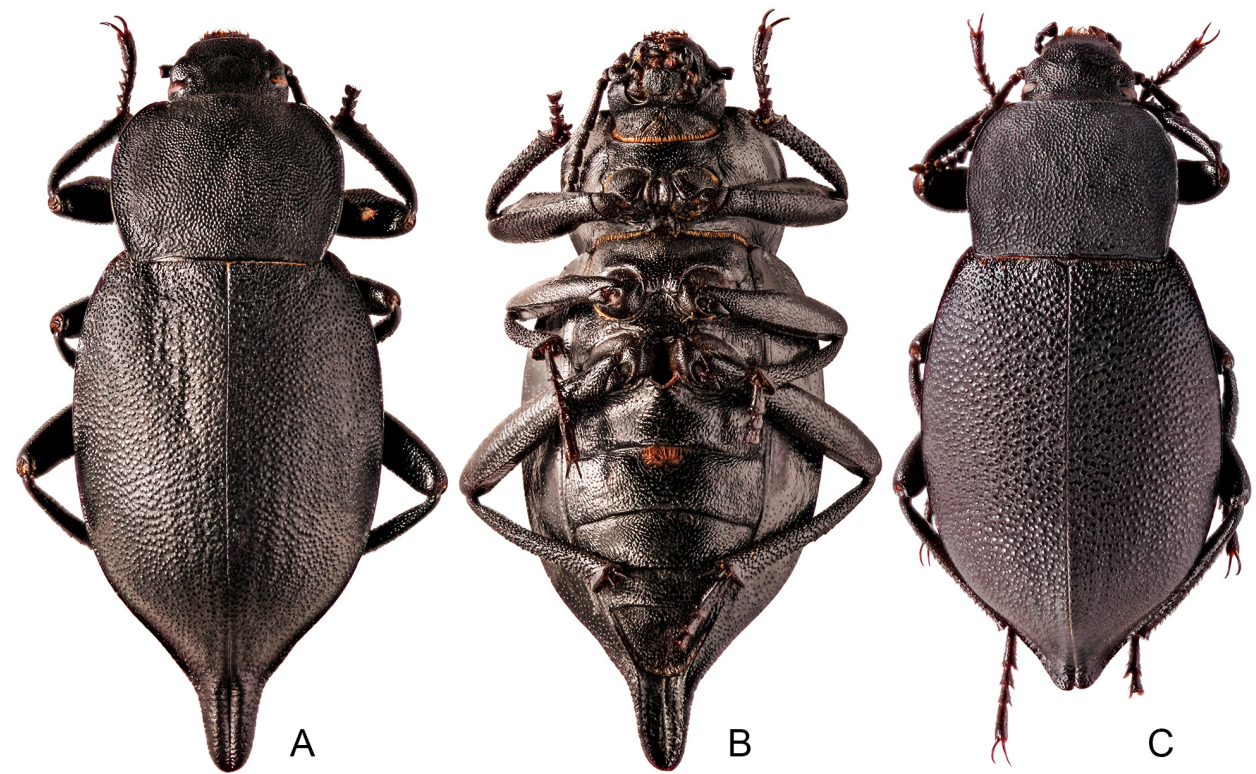

Fig. 16. B. granulata psammophila, habitus. $\mathrm{A}, \mathrm{B}=\widehat{\mathrm{O}} ; \mathrm{C}=q ; \mathrm{A}, \mathrm{C}=$ dorsal view; $\mathrm{B}=$ ventral view 
Blaps granulata obliterata Ménétriés, 1849

(Fig. 17)

Ménétriés, 1849: 236 (“Turcomanie”); Skopin, 1966: 337; Skopin, 1968: 85.

Material examined. 1 ㅇ, ‘Turkestan // Blaps caudata / Turkestan / ii.20’ (NMP); 1 ○, 'Syr Daria / Bang-Haas' (NMP); 1 §’, ‘Western Balkhash Lake region / 120 km W Baital village / 15.ix.1964 / leg. N. Skopin' [in Cyrillics] (ZIN); 1 + , 'Kyrgyz Range / Shamsi pass / viii.1958 / leg. Pivovarov' [in Cyrillics] (ZIN).

Distribution. Kazakhstan (LöBL et al. 2008).

Regional distribution. Muyunkum Desert, to the west and south of Balkhash Lake (Baital), the lower course of the Ili River (Sкорім 1966, 1968).

Blaps granulata turcomana Fischer von Waldheim, 1843

(Fig. 18)

Fischer von Waldheim, 1844: 88; Gebler, 1844: 103 ("Blaps caudata Gebler, 1844"); Skopin, 1960: 50 ("B. caudata") (larva); Skopin, 1961: 189 ("B. caudata"); Skopin, 1966: 337 ("B. caudata"); Skopin, 1968: 85 ("B. caudata"); Ren et al. 2016: 121.
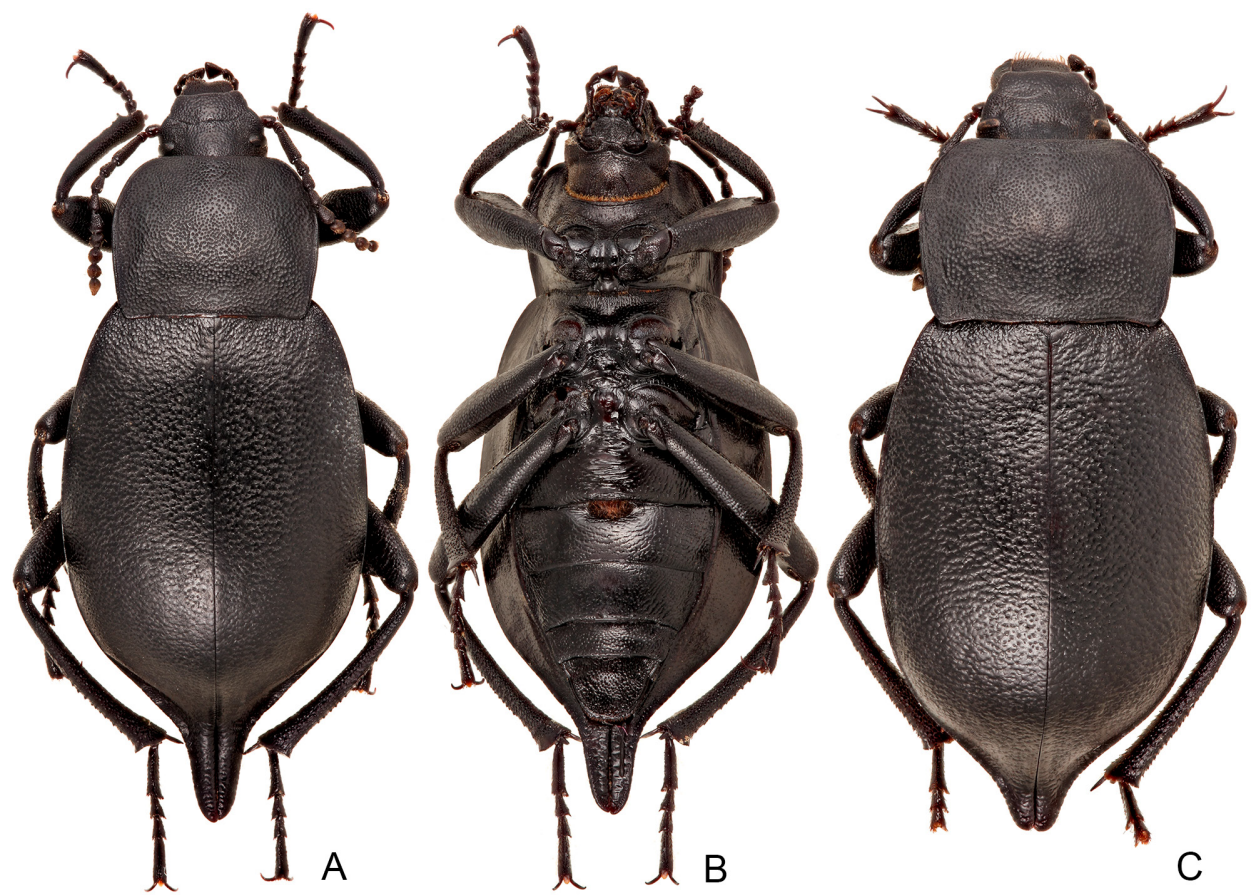

Fig. 17. B. granulata obliterata, habitus. $A, B=\widehat{O} ; C=\rho ; A, C=$ dorsal view; $B=$ ventral view 
Material examined (ZIN). 1 ऽ, 1 \&, 'Southeast Kazakhstan / Alma-Ata Region / Bay-

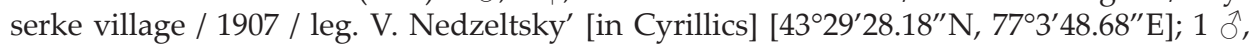
'Southeast Kazakhstan / Alma-Ata Region / Alma-Ata / 25.iv.1950 / leg. N.G. Skopin' [in

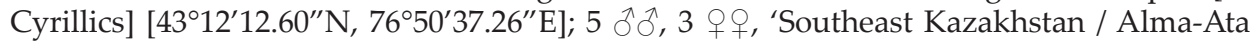
Region / Anrahai Mountains / canyon of Kopaly River / 14.v.1909 / leg. Nedovpukov' [in

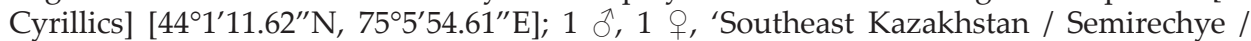
Kurdayskiy pass / h = $1100 \mathrm{~m} / 16 . v .1907$ / leg. A. Jakobson' [in Cyrillics] [4316'23.25”N,

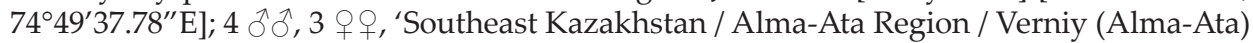

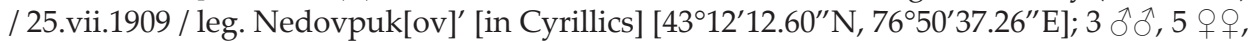
'Southeast Kazakhstan / Alma-Ata Region / Jambyl region / Korday village / 07.vii.1906 /

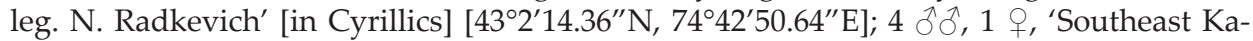
zakhstan / Alma-Ata Region / Alma-Ata city suburbs / 19.vi.1909 / leg. Nedovpuk[ov]' [in

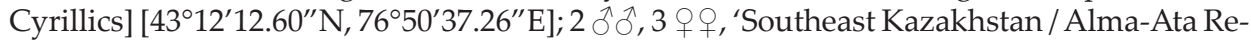
gion / Anrahai Mountains / Almalysai River / 10-14.v.1909 / leg. Nedovpukov' [in Cyrillics]

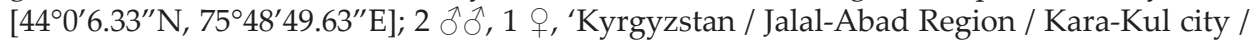

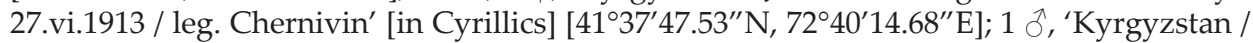
Naryn Region / Kokomeren River / 18-19.v.1914 / leg. Mikhalevskaya' [in Cyrillics].

Distribution. Kazakhstan, Uzbekistan, Kyrgyzstan, China (Nei Mongol and Xinjiang provinces) (LöBl et al. 2008, REN et al. 2016).

Regional distribution. The distribution overlaps with that of the nominotypical subspecies (SKOPIN 1968).

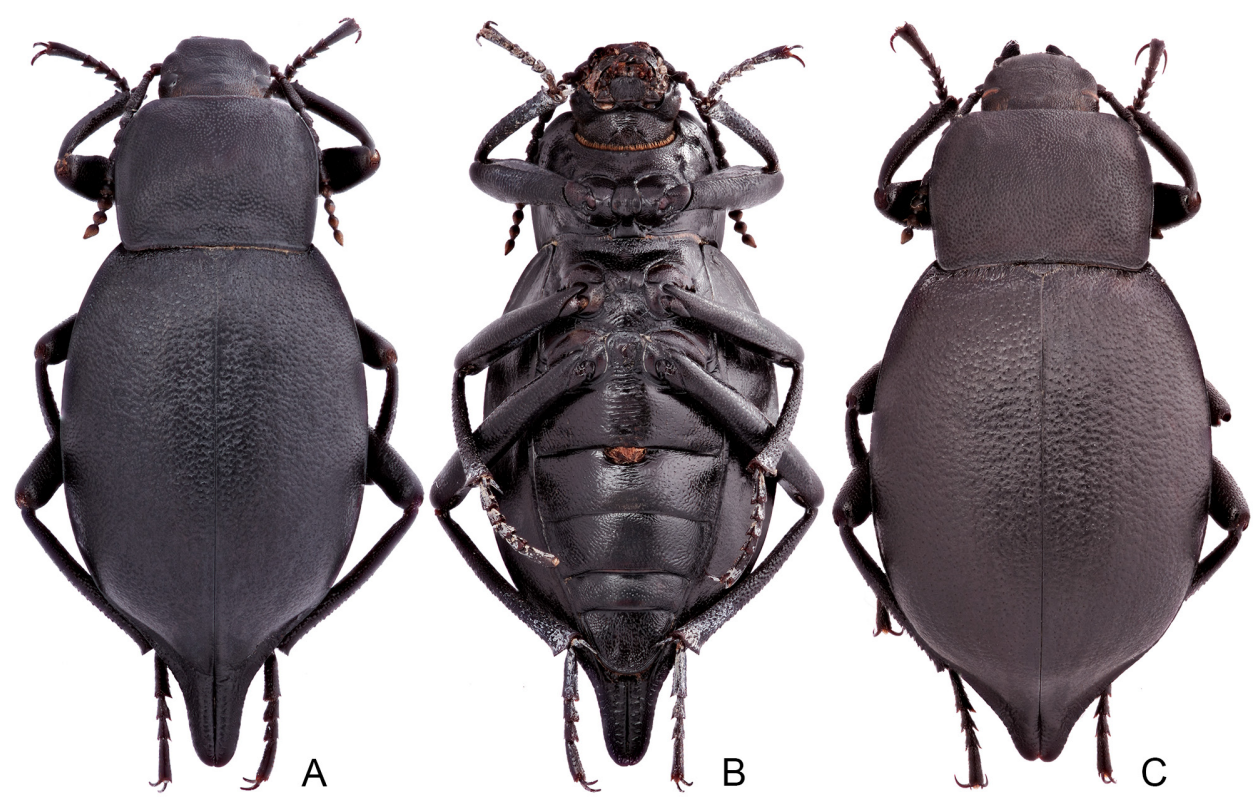

Fig. 18. B. granulata turcomana, habitus. $A, B=\widehat{C} ; C=q ; A, C=$ dorsal view; $B=$ ventral view 


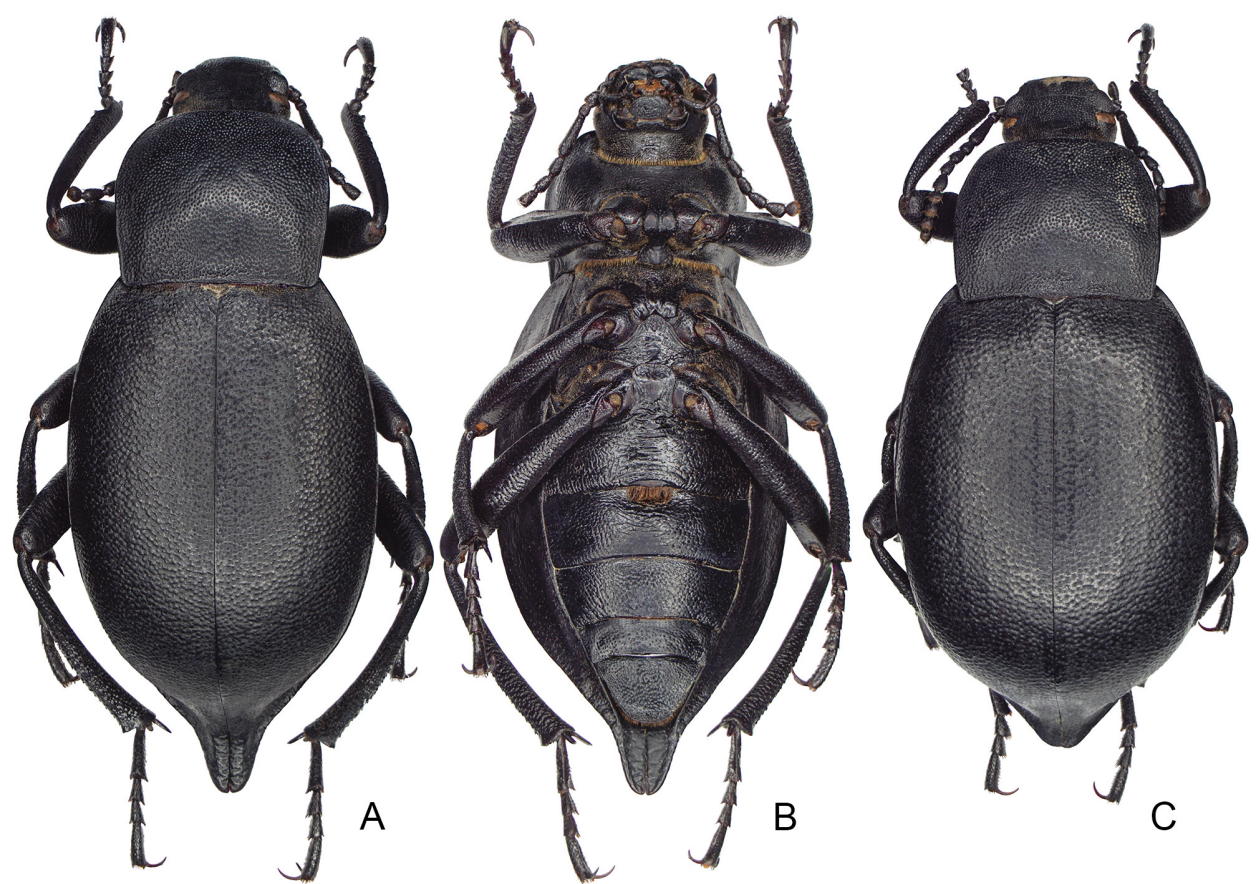

Fig. 19. B. granulipennis, habitus. $A, B=O^{\lambda} ; C=q ; A, C=$ dorsal view; $B=$ ventral view
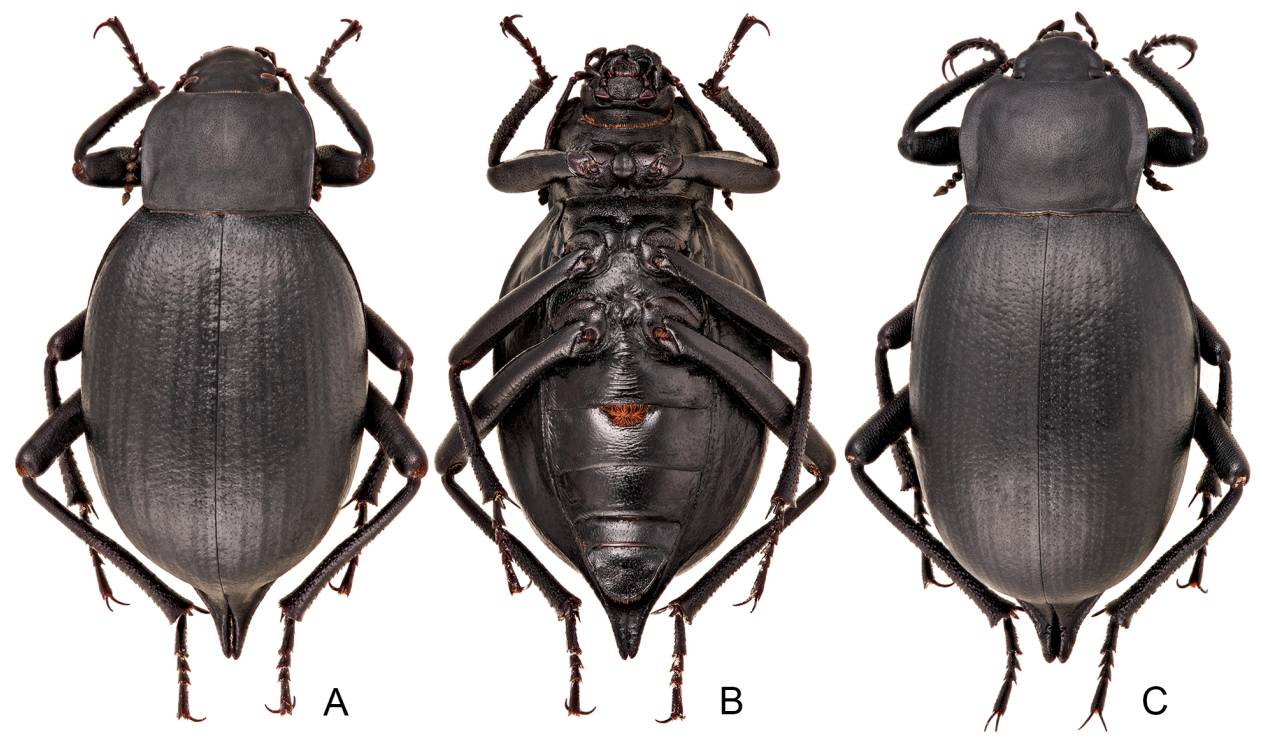

Fig. 20. $B$. holconota, habitus. $\mathrm{A}, \mathrm{B}=\widehat{\delta} ; \mathrm{C}=\rho$; $\mathrm{A}, \mathrm{C}=$ dorsal view; $\mathrm{B}=$ ventral view 


\section{Blaps granulipennis Skopin, 1966}

(Figs 19, 27A)

Skopin, 1966: 337.

Type material examined (ZIN). Holotype $(\precsim)$, allotype $(ð)$ and paratypes (2ᄋㅇ): 'Eastern Kazakhstan / east coast of Lake Alakol / $20 \mathrm{~km}$ north of Uzynbulak village / 06.vii.1965 / leg. N.G. Skopin' [in Cyrillics] [4554'44.73”N, 82¹0’50.51"E].

Comments. Skopin (1966) described this species (after study of six specimens available to him), but did not indicate the number of males and females in the description. There are four specimens in the collection of ZIN: the holotype, the allotype (male!) and 2 paratypes.

Distribution. Kazakhstan: the area surrounding Alakol Lake and Zhalanashkol Lake (SкоріN 1966).

\section{Blaps holconota Fischer von Waldheim, 1844 (Figs 20, 21C,D)}

Fischer von Waldheim, 1844: 71 (Desertis Kirgisorum); Skopin, 1960: 57 (larva); Skopin, 1961: 192; Skopin, 1968: 86; Arnoldi \& Medvedev, 1969: 403; Medvedev \& Nepesova, 1985: 119 (Blaps scutellata); Chigray et al., 2016: 9.

Material examined (ZIN). $2 \widehat{\widehat{\partial}}$, 'Southeast Kazakhstan / Jambyl Region / Moyyn-

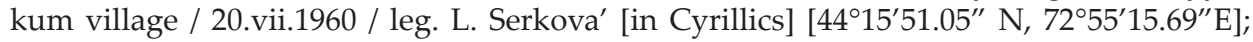
$1 \hat{\delta}$, 'Southeast Kazakhstan / Jambyl Region / Muyunkum village / 22.iv.1954 / leg. L.

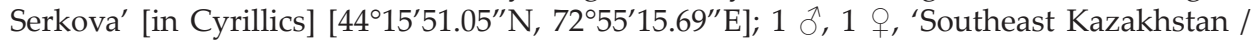
Alma-Ata Region / 40 km south-west of Zharkent city / 26.iv.1965 / leg. N.G. Skopin' [in

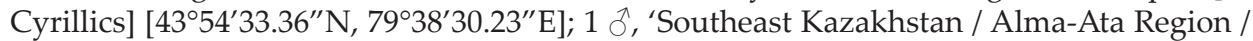
Sasykbulak spring / Ulken-Bugutty mountains / 29.vi.1960 / leg. N. Skopin' [in Cyrillics] [4332'25.36"N, 794'15.09" E]; 1 ㅇ, 'Southeast Kazakhstan / Alma-Ata Region / Kokishbay village / lower course of Ili River / Rondy duct / 16.ix.1948 / leg. D. Aleksandrov' [in Cyrillics]

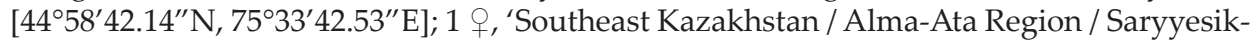

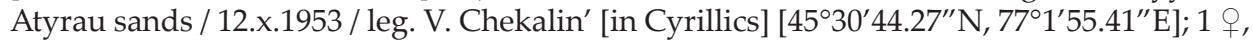
Southeast Kazakhstan / Alma-Ata Region / Sarybulak village / 06.vii.1907 / leg. A. Jakob-

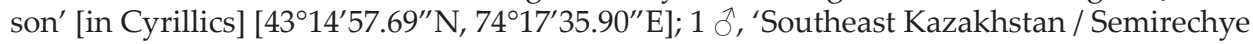
Region / Dzharkentskiy uyezd / Ili River / 18.iv.1906 / leg. V. Ryunbeyl' [in Cyrillics]; 1 ○े, 3 우, 'Semirechye province. / Galford Mountain pass' [in Cyrillics].

Comments. Arnoldi and Medvedev (1969) distinguished two species: Blaps holconota and Blaps scutellata Fischer von Waldheim, 1844. These names are listed in the catalogue of LöвL et al. (2008) as concerning different species, but the authors of this paper use the name Blaps holconota because didn't find distinct differences between these two taxa (CHIGRAy et al. 2016: 9). 


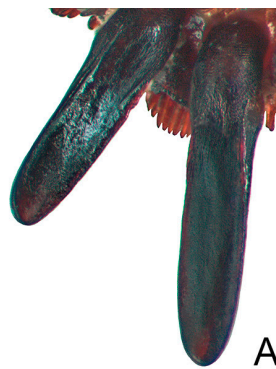

A
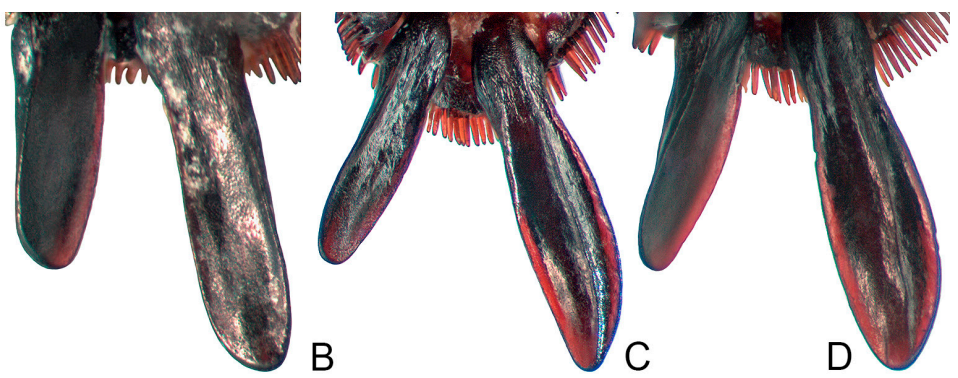

Fig. 21. The spurs. $\mathrm{A}, \mathrm{B}=\mathrm{B}$. deplanata; $\mathrm{C}, \mathrm{D}=\mathrm{B}$. holconota; $\mathrm{A}, \mathrm{C}=$ the spurs on the mesotibiae; $\mathrm{B}, \mathrm{D}=$ the spurs on the metatibiae

Distribution. Northern and Southern Kazakhstan, Uzbekistan, Turkmenistan, Afghanistan (Sкорім 1961, Arnoldi \& Medvedev 1969, Löвl et al. 2008).

Regional distribution. Desert areas of South Kazakhstan (SKopin 1961, Arnoldi \& Medvedev 1969).

\section{Blaps inflexa Zoubkoff, 1833}

Zoubkoff, 1833: 331; Seidlitz, 1893: 292; Skopin, 1960: 52 (larva); Skopin, 1968: 84; Medvedev \& Nepesova, 1985: 120; Chigray et al., 2016: 6. (see figures in: Chigray et al. 2016: fig. 10)

Material examined (ZIN). 1 o, 'Kazakhstan / middle of Syr Darya River / Kyzylorda /

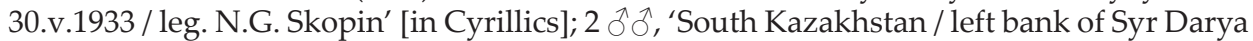
River / Tugay / between Shardara and Kok-su / 11.v.1964 / leg. N.G. Skopin' [in Cyrillics].

Distribution. Kazakhstan, Uzbekistan (from Namangan to Amu Darya River delta), Turkmenistan (Medvedev \& Nepesova 1985 (Amu Darya River delta); LöвL et al. 2008).

Regional distribution. The species is distributed in Syr Darya River valley (SKорIN 1968).

Blaps kadyrbekovi Medvedev, 2004

Medvedev, 2004: 570; Chigray et al., 2016: 6. (see figures in: Chigray et al. 2016: figs 9D-F)

Type material examined (ZIN). Holotype and paratypes from both localities were studied (Medvedev 2004).

Distribution. Kazakhstan: Atyrau Region (Kulsary), North Aral Sea coast (Butakov gulf) (Medvedev 2004). 
Blaps lethifera lethifera Marsham, 1802

Marsham, 1802: 479; Faldermann, 1837: 50 ("Blaps anthracina"); Motschulsky, 1845: 68 ("Blaps robusta"); Skopin, 1960: 53 (larva); Arnoldi \& Medvedev, 1969: 403; Chigray et al., 2016: 7. (see figures in: Chigray et al. 2016: figs 11A-C)

Type material examined (ZIN). LeстотүPe of Blaps robusta (designated by AвDURAKHMANOv \& NABOzHenко 2011): 9 , 'Lehmann // Blaps robusta Motsch // Lectotypus / Blaps robusta / des. Abdurakhmanov \& Nabozhenko'. LестотүPE of Blaps anthracina (designated by Авduraknmanov \& Nabozhenko 2011): + , 'Caucas. // anthracina Fald. Caucas. // Lectotypus / Blaps anthracina / des. Abdurakhmanov \& Nabozhenko'.

Material examined (ZIN). More than 60 specimens from Western Kazakhstan Region.

Distribution. Europe (everywhere, north to Scandinavia and the European part of Russia), North Africa, the Middle East, North Caucasus, Western Siberia (Abdurakhmanov \& Nabozhenko 2011), Western (Chigray et al. 2016), North and NE Kazakhstan (Sкоріn 1960), China: Xijiang (Ren at al. 2016).

Regional distribution. Karaganda Region (SKopin 1960, Arnoldi \& MedVEDEV 1969).

\section{Blaps motschulskiana Bogatchev, 1947}

(Figs 22, 36A)

Motschulsky, 1845: 65 ("Blaps gigantea"); Seidlitz, 1893: 268 ("B. gigantea"); BogAtchev, 1947: 515 (B. motschulskiana, new name for B. gigantea Motschulsky, 1845 nec B. gigantea L. Petagna, 1819); Skopin, 1968: 87 (“Lithoblaps gigantea").
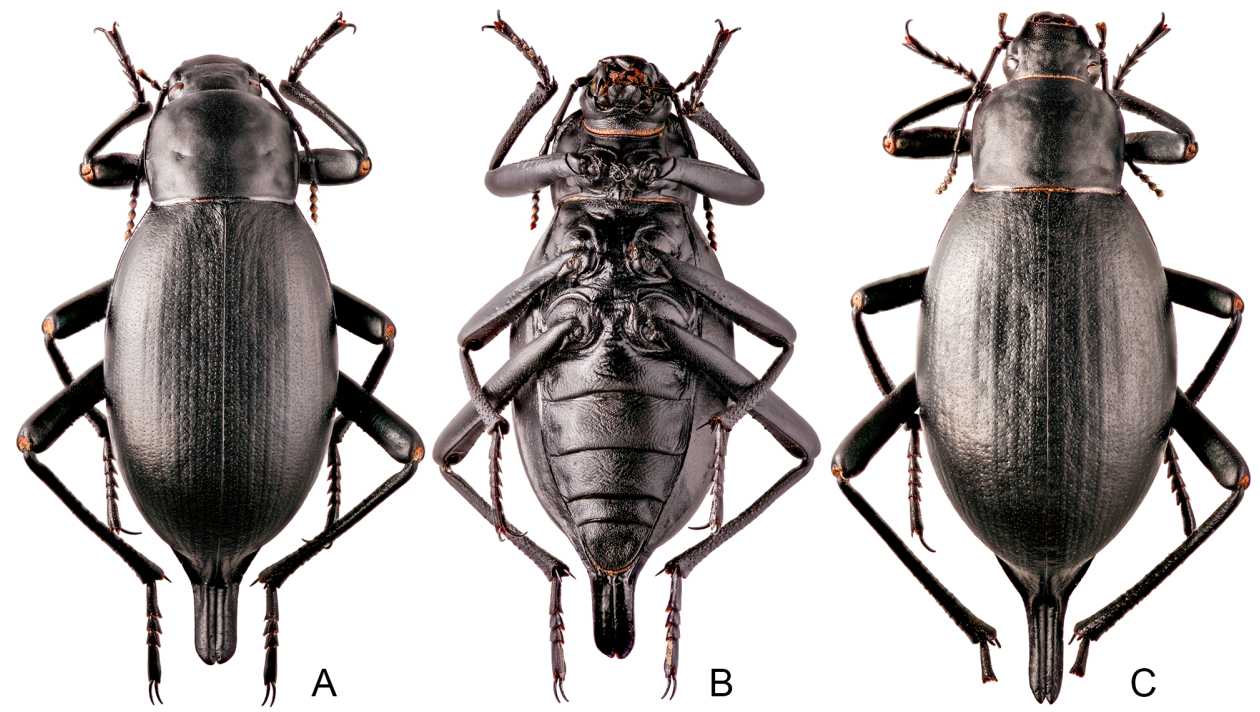

Fig. 22. $B$. motschulskiana, habitus. $A, B=\widehat{O} ; C=$; $A, C=$ dorsal view; $B=$ ventral view 
Type material examined (ZIN). Lectotype of Blaps gigantea Motschulsky, 1845 is designated here: $\hat{\delta}$, 'Blaps gigantea Motschulsky / Tekke. // Lectotypus / Blaps gigantea Motschulsky, 1845 / des. I.A. Chigray'.

Material examined (ZIN). 1 đ’, ‘Turkmenistan / Karahan / 15.v.1976 / leg. G. Medve$\operatorname{dev}^{\prime}$ [in Cyrillics]; 1 ㅇ, ‘Turkestan'; 1 q 'Turkmenistan / Trans-Caspian Region / Ashgabat / 3.v.1889 / leg. A. Semenov' [in Cyrillics]; 1 đ’ 'Turkmenistan / Trans-Caspian Region / Ashgabat / 2.v.1889 / leg. A. Semenov' [in Cyrillics]; 2 + $q$ 'Trans-Caspi G. / Turcmenien / leg. E. König'; 1 q 'Turkmenistan / Ashgabat / 1986 / leg. Varentsev' [in Cyrillics].

Distribution. Kazakhstan (SKopIN 1968), Turkmenistan, Afghanistan (LöвL et al. 2008).

Regional distribution. Southeastern parts of Kyzylkum desert and Syr Darya River near the border of Uzbekistan (SKOPIN 1968).

\section{Blaps nitida Fischer von Waldheim, 1844}

(Fig. 23)

Fischer von Waldheim 1844: 96; Seidlitz, 1893: 280; Skopin, 1961: 190.

Material examined (ZIN). 1 ก, 1 ㅇ, ‘Central Kazakhstan / Karaganda Region / 30 km west of Gulshad village [46³7'27.91”N, 7357'55.11”E] / 17.ix.1964 / leg. N.G. Skopin' [in Cyrillics].
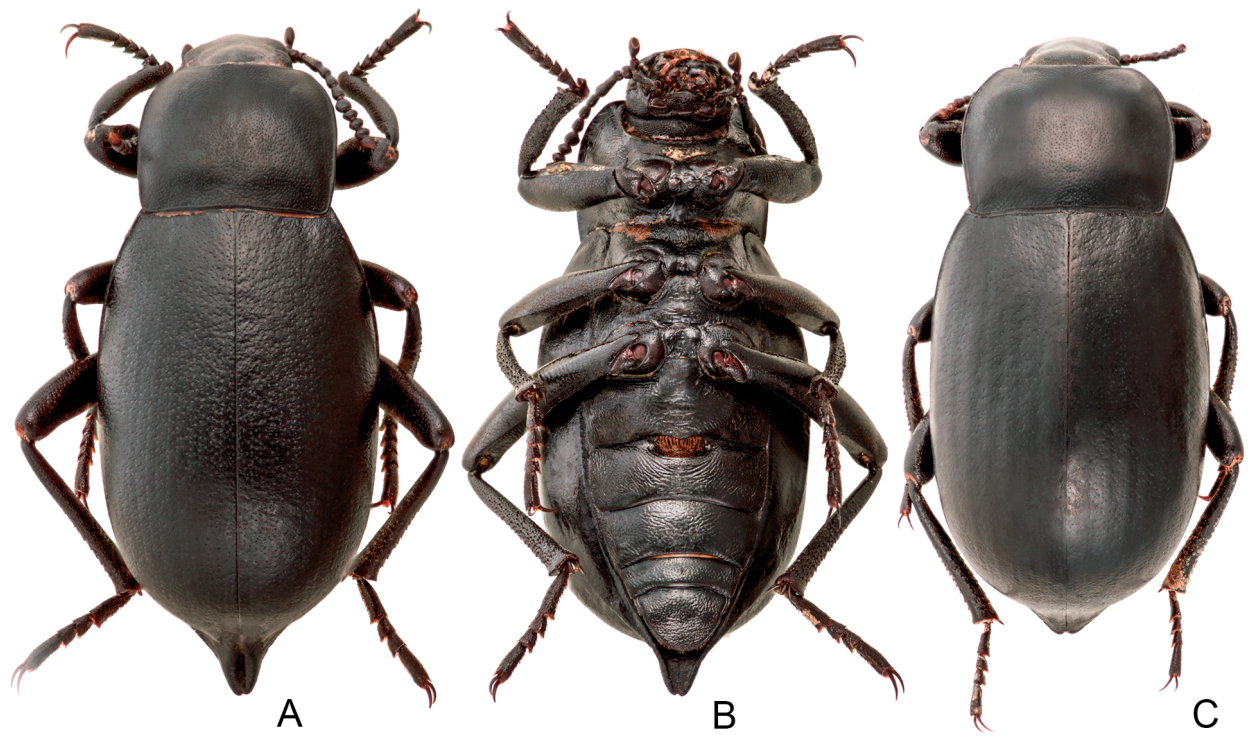

Fig. 23. $B$. nitida, habitus. $A, B=\widehat{B} ; C=+; A, C=$ dorsal view; $B=$ ventral view 
Comments. Sкоргл (1973) discussed the taxonomic position of Blaps nitida Fischer von Waldheim, 1844 and the very variable B. lethifera Marsham, 1802. The first taxon is listed as a subspecies of $B$. lethifera in the card index and the collection of ZIN, but as a valid species in the Catalogue of Palaearctic Coleoptera (LöвL et al. 2008). The status of B. nitida can be clarified after the study of type specimens. In the subsequent key, $B$. nitida and B. lethifera are given in the same key couplet. Arnoldi and Medvedev (1969) did not mention B. nitida for Central Kazakhstan.

Distribution. Kazakhstan, Russia (Western Siberia) (LöвL et al. 2008).

Regional distribution. Central and Southeast Kazakhstan (SкоріN 1961).

\section{Blaps parvicollis parvicollis Zoubkoff, 1829}

Zoubkoff, 1829: 160; Seidlitz, 1893: 281; Skopin, 1960: 54 (larva); Arnoldi \& Medvedev, 1969: 404; Chigray et al. 2016: 6. (see figures in: CHigray et al. 2016: figs 9A-C)

Material examined. 1 + , ‘Kazakhstan / Aral Sea / Barsa-Kelmes Island [a former island of Aral Sea] / 20.vi.1940 / leg. Zvorygin' [in Cyrillics].

Additional material examined. More than 50 specimens from Western Kazakhstan.

Comments. The records for Karaganda and Jambyl regions (ARNOLDI \& Medvedev 1969) are erroneous and probably belong to the subspecies Blaps parvicollis quadricollis Ballion, 1878. Differentiation of the subspecies by females is difficult. The female studied from Barsakelmes Reserve (the western part of Southern Kazakhstan) has the body shape and the structure of genitalia similar to those of the nominotypical subspecies from Western Kazakhstan. The authors of this paper have not seen any male of the nominotypical subspecies from South Kazakhstan.

Distribution. South of European part of Russia, Azerbaijan (Apsheron), Kazakhstan (Abdurakhmanov \& Nabozhenko 2011)

Regional distribution. The Barsakelmes Nature Reserve (a former island in the Aral Sea).

Blaps parvicollis quadricollis Ballion, 1878

(Fig. 24)

Ballion, 1878: 304; Skopin, 1973: 868 (B. parvicollis, subsp.).

= subcordata Seidlitz, 1893: 306; Semenov Tian-Shansky \& Bogatchev 1936: 567; Skopin, 1960: 54 (larva); Skopin, 1961: 190 (B. parvicollis, subsp.); Skopin, 1968: 85 (B. parvicollis, subsp.).

Type material examined (NHM). LeстотүPE of Blaps subcordata (designated by Skopin): ${ }^{\pi}$, 'Haberhauer / Turkestan / 89 // Lectotypus / Blaps subcordata Soll. / N. Skopin de- 
sign. / 1978 // Blaps parvicollis quadricollis Ball. / N. Skopin det. / 1978'. PARAlectotypes: 1 đ̊, 1 + , 'Haberhauer / Turkestan / 89'.

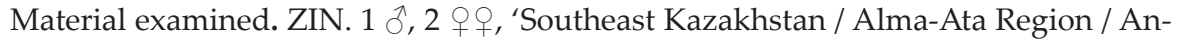
rahai Mountains / canyon of Kopaly River / 16-19.v.1909 / leg. Nedovpuk[ov]' [in Cyril-

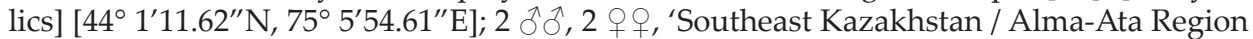
/ canyon of Kopaly River / 19.v.1909 / leg. Nedovpuk[ov]' [in Cyrillics] [44 1'11.62"N, 75 5'54.61"E]; 2 우어, 'Southeast Kazakhstan / Jambyl region / Akyr-Tyube village / 25.v.1931

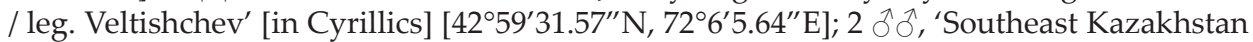
/ Alma-Ata Region / Ili-Karatal along Turkestan-Siberian Railway / 05.vi.1930 / leg. V. Kizeritskiy' [in Cyrillics]; 1 ô, 1 ㅇ, 'Southeast Kazakhstan / Semirechye Region / Kunkuzskaya Upland (Altyn-Emel Range) to north of Aladan / iv.1879 / leg. E. Regel'; 1 o, 1 , 'Southeast Kazakhstan / Semirechie / upper reaches of River Bota-Boruly / 20.v.1909 / leg. Nedovpuk[ov]' [in Cyrillics]; 1 +, 'Southeast Kazakhstan / Alma-Ata Region / Alma-Ata

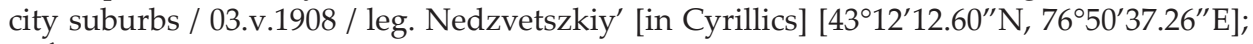
1 ㄱ, 2 우, 'Southeast Kazakhstan / env. of Jambyl / Merke village / iv.1910 / leg. E. Fis-

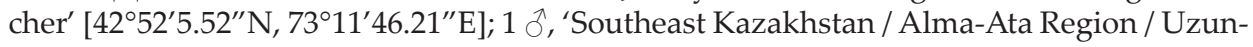

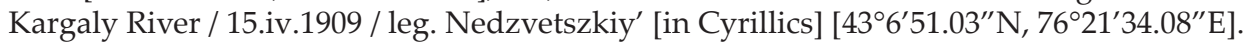

Comments. SкоріN (1960) suggested that Blaps parvicollis subcordata Seidlitz, 1893 could be the eastern subspecies of B. parvicollis, and indicated that the larvae of both subspecies are very similar in a structure. Later, SкоріN (1973) synonymized B. parvicollis subcordata with B. parvicollis quadricollis Ballion, 1878 and pointed to the error of SEMEnov Tian-SHAnsky and Bogatchev (1936), who noted that B. quadricollis is indistinguishable from B. pterosticha
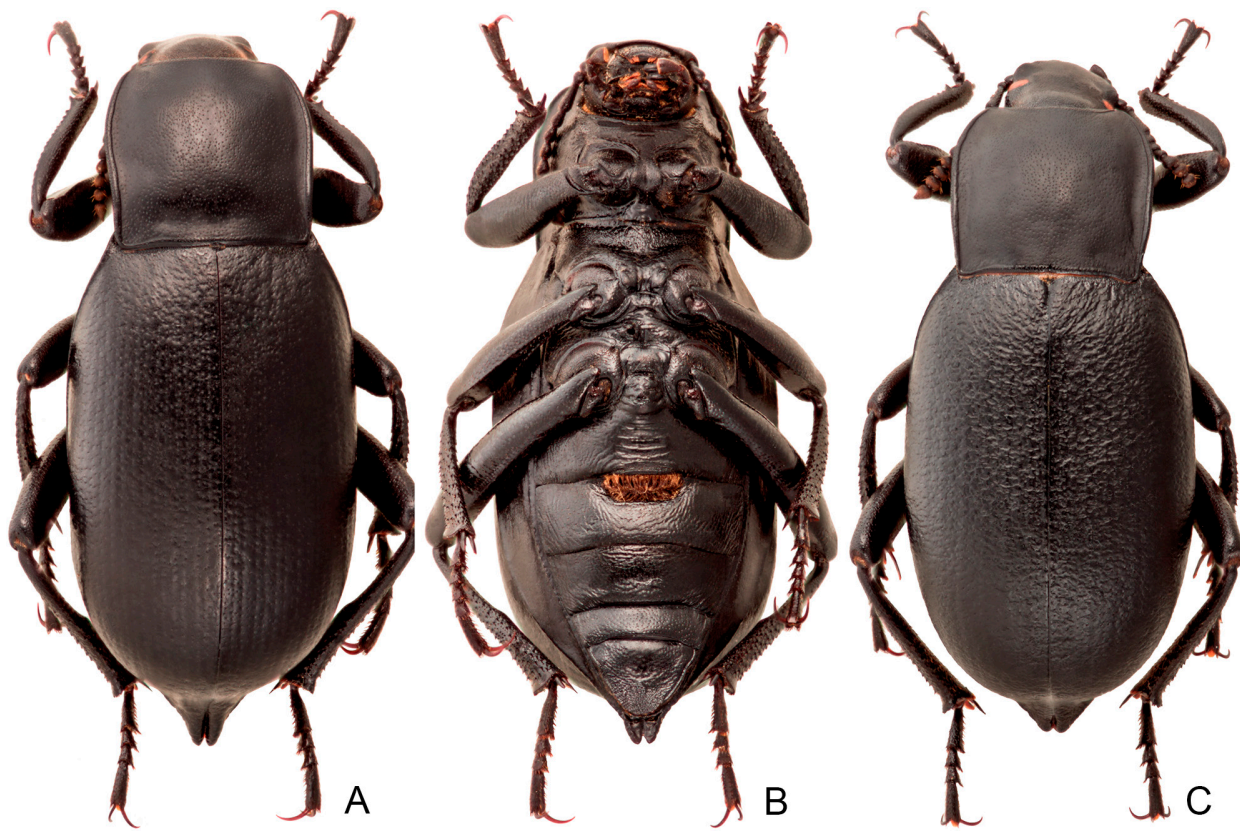

Fig. 24. B. parvicollis quadricollis, habitus. $\mathrm{A}, \mathrm{B}=\mathrm{O} ; \mathrm{C}=\rho$ + $\mathrm{A}, \mathrm{C}=$ dorsal view; $\mathrm{B}=$ ventral view 
Fischer von Waldheim, 1844. LöвL et al. (2008) erroneously listed Blaps parvicollis subcordata as a separate species and B. parvicollis quadricollis as a junior synonym of $B$. pterosticha.

Blaps parvicollis quadricollis differs from B. pterosticha in smaller body size, coarser pronotal bead, male metatibia with thickening in apical half and subglobular reservoirs of spermatheca. Based on the more elongated pronotum and elytra, coarser and thicker pronotal bead and male metatibia with thickening in the apical half (Figs 25A, B), structures of male and female genitalia and also presence of populations with intermediate characters in intergradation zones, it is thought that B. p. quadricollis is a subspecies of B. parvicollis. Separation of females is difficult, as the body shape (in particular structure of pronotum and elytra) and the structure of spermatheca are rather variable.
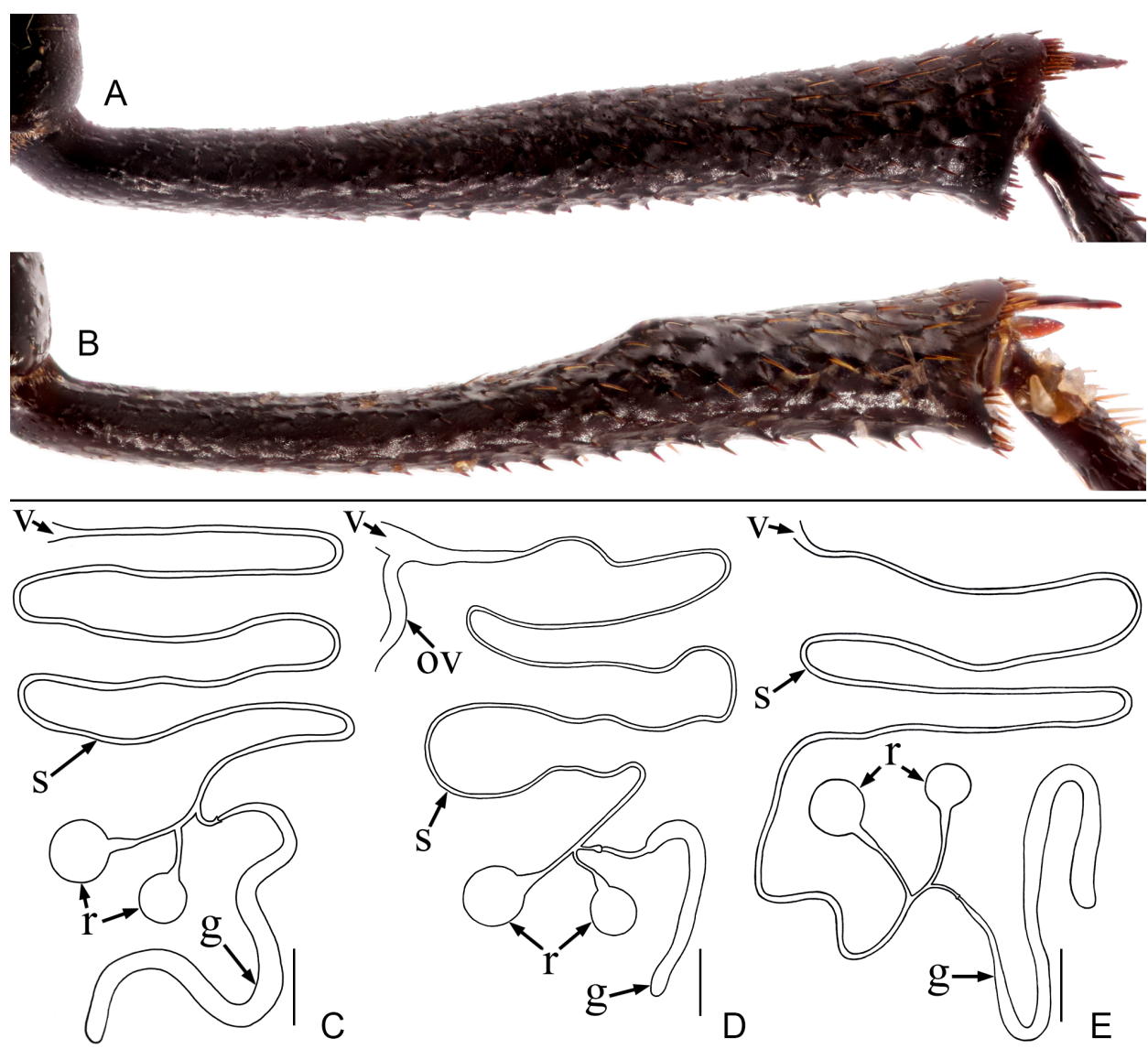

Fig. 25. Subspecies B. parvicollis, details of structure. A - B. parvicollis parvicollis, B - B. parvicollis quadricollis; A, B - male metatibia; C-E - the variability of genital ducts of females. (ov - oviduct; $\mathrm{v}$ - vagina, $\mathrm{s}$ - basal duct of spermatheca, $\mathrm{r}$ - reservoirs, $\mathrm{g}$ - accessory gland of spermatheca). Scale bars $=1 \mathrm{~mm}$ 
Some authors (Medvedev 2001, Chigray et al. 2016) illustrated female genital ducts of $B$. $p$. parvicollis. Recent studies revealed that the genital ducts of each of both subspecies of B. parvicollis in Southern Kazakhstan are represented by three types: 1) bases of both reservoirs of the spermatheca are joined in a common duct at a distance from the basal duct (Fig. 25C); 2) bases of reservoirs of the spermatheca are joined and do not form a common tube before the basal duct (Fig. 25D); 3) bases of reservoirs of the spermatheca are separately joined with the basal duct (Fig. 25E).

Distribution. The subspecies is widely distributed in the southern zone of Kazakhstan (Sкорім 1968).

\section{Blaps pruinosa Eversmann, 1833}

Eversmann, 1833: 53; Motschulsky, 1860: 532 ("Rhizoblaps"); Seidlitz, 1893: 268; Skopin, 1960: 58 ("Lithoblaps") (larva); Skopin, 1961: 58 ("Lithoblaps"): 192; Skopin, 1968: 87 ("Lithoblaps"); Arnoldi \& Medvedev, 1969: 404; Medvedev \& Nepesova 1985: 115; Chigray et al. 2016: 12. (see figures in: CHigray et al. 2016: figs 13A-C)

Type material examined (ZIN). Lectotype of Blaps pruinosa (designated by AвDurAкнмanov \& Nabozhenко 2011): $\widehat{0}$, 'Kirgis Step // Lehmann // Blaps pruinosa Eversm. Kirgs. Desert // golden square // Lectotypus / Blaps pruinosa Eversmann / des. Abdurakhmanov \& Nabozhenko'. Lectotype of Blaps rorulenta Motschulsky, 1845 (designated by AвdurAKHMAnov \& Nabozhenko 2011): ㅇ, 'rorulenta // Rhisoblaps rorulenta Motch. Songoria // c. Motschulsky // golden square // Lectotypus / Blaps rorulenta 1845 / des. Abdurakhmanov \& Nabozhenko'.

Material examined (ZIN). $1 \hat{\sigma}, 1$ q, 'Southeast Kazakhstan / Alma-Ata Region / 40 km south-west of Zharkent village / 26.iv.1965 / leg. N.G. Skopin' [in Cyrillics] [4359'24.43”'N, 79०34'3.92"E].

Additional material examined (ZIN). More than 50 specimens from the Caspian Depression.

Distribution. Southeast of the European part of Russia, Kazakhstan (from the Caspian sea to southern foothills of Tarbagatay Mts.), Uzbekistan, Turkmenistan, Tajikistan (Medvedev \& Nepesova 1985, Abdurakhmanov \& NABozhenko 2011).

Regional distribution. The species is widely distributed in the central (Arnoldi \& Medvedev 1969) and southern zones of Kazakhstan to eastern Balkhash Lake (SKOPIN 1961).

\section{Blaps pterosticha Fischer von Waldheim, 1844} (Figs 26, 27C)

Fischer von Waldheim, 1844: 93; Seidlitz, 1893: 286 ("Turkestan"); Skopin, 1960: 55 (larva); Skopin, 1961: 191; Skopin, 1968: 86; Ren et al., 2016: 153. 


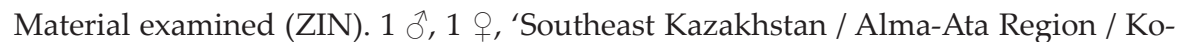

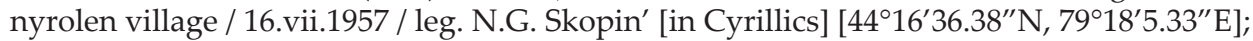
1 స, 1 ㅇ, 'Southeast Kazakhstan / Alma-Ata Region / 40 km south-west of Zharket village

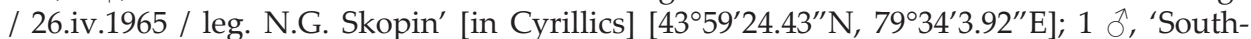
east Kazakhstan / Alma-Ata Region / 30 km north of Taldykorgan city / 07.ix.1962 / leg.

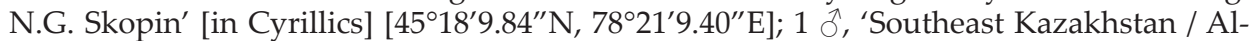
ma-Ata Region / Kurtogay natural boundary / 15.vii.1959 / leg. N.G. Skopin' [in Cyril-

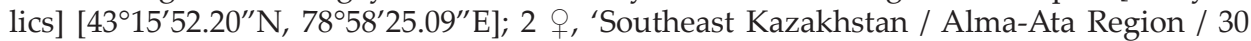
$\mathrm{km}$ south of Dubun village / 10.vi.1969 / leg. N.G. Skopin' [in Cyrillics] [43²6'5.87”N, 80¹1'13.00"E]; 1 오 'Southeast Kazakhstan / Alma-Ata Region / Kurtogay natural bound-

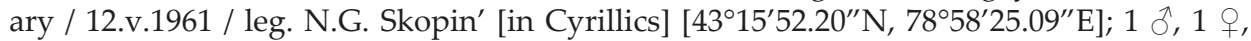
'Southeast Kazakhstan / Alma-Ata Region / 40 km north of Zharkent village / 14.v.1961

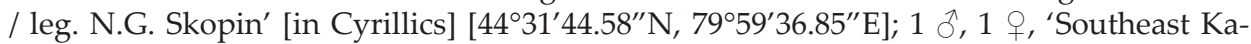
zakhstan / Alma-Ata Region / Saryzhaz village / 25.vi.1966 / leg. N.G. Skopin' [in Cyrillics] [42 54'31.02"N, 79³5'49.30”E]; 1 ㅇ, 'Southeast Kazakhstan / Alma-Ata Region / Kapcha-

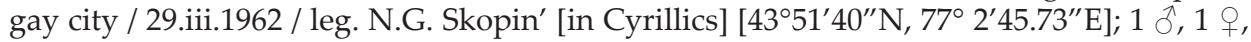
'Southeast Kazakhstan / Alma-Ata Region / Zharkent village / 12.v.1961 / leg. N.G. Skopin' [in Cyrillics] [44 9'24.73"N, 7959'53.04"E].

Comments. SKopin (1961) showed that Blaps pterosticha and B. tenuicauda Seidlitz, 1893 are morphologically similar species and their lifestyles are the
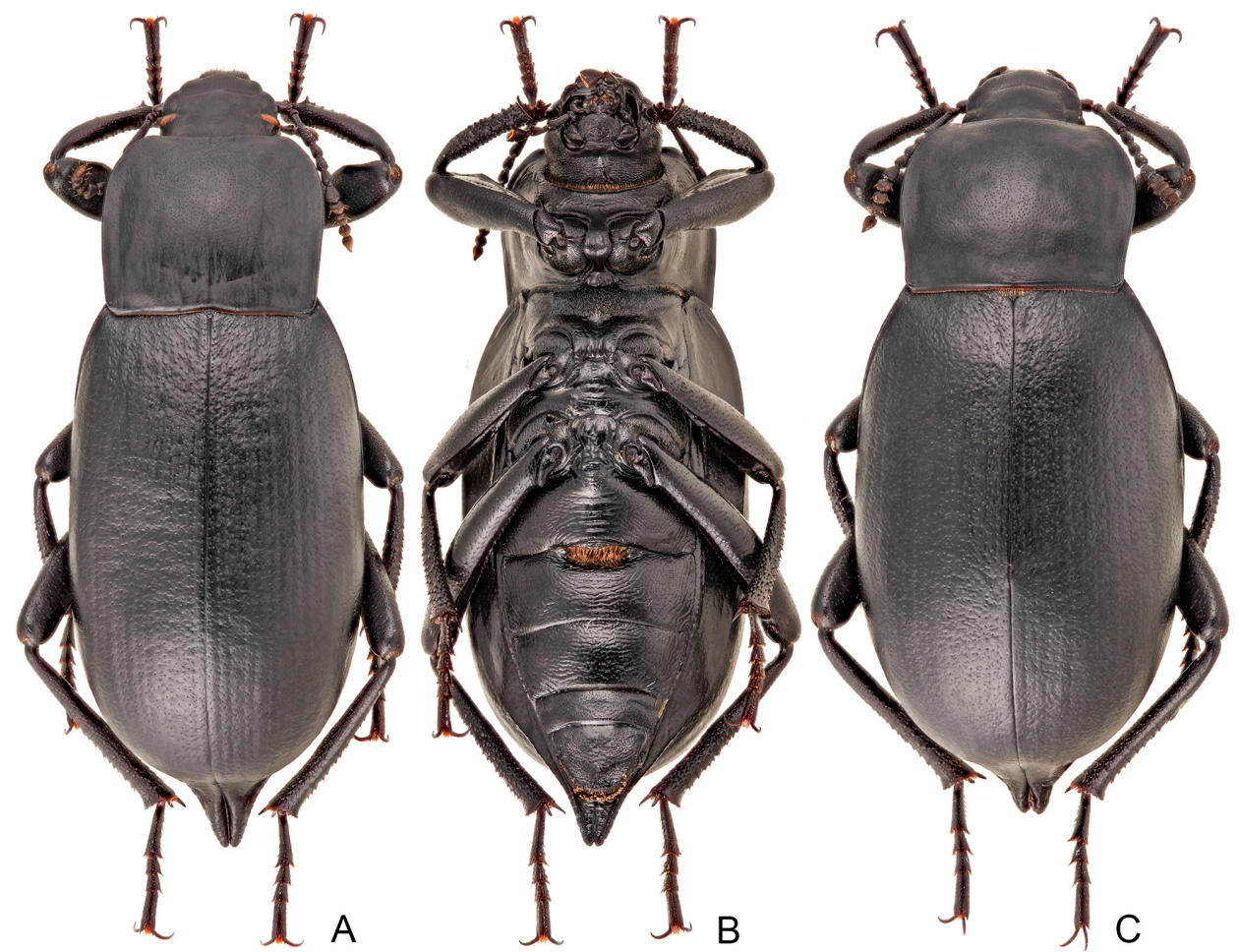

Fig. 26. B. pterosticha, habitus. $\mathrm{A}, \mathrm{B}-\widehat{O} ; \mathrm{C}=q ; \mathrm{A}, \mathrm{C}=$ dorsal view; $\mathrm{B}=$ ventral view 


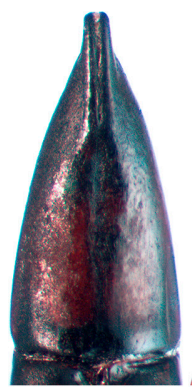

A

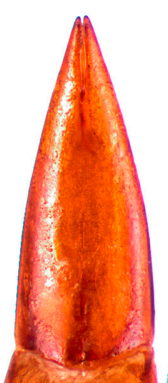

B

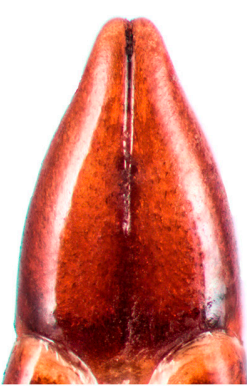

C

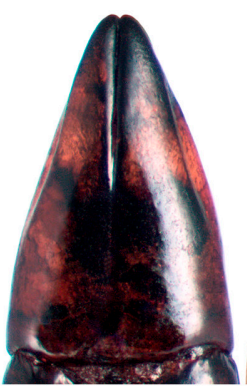

$\mathrm{D}$

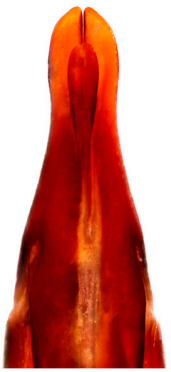

E

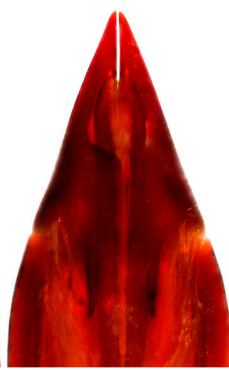

$\mathrm{F}$

Fig. 27. Apical piece of aedeagus (parameres). $A=B$. granulipennis; $B=B$. granulata granulata $; \mathrm{C}=$ B. pterosticha $; \mathrm{D}=$ B. evanida $; \mathrm{E}=$ B. faustii; $\mathrm{F}=$ B. turcomanorum

same; he suggested that $B$. tenuicauda is only a form of B. pterosticha. Recent studies confirmed this opinion based on the absence of any significant external and internal structural differences between the specimens formerly considered as these two "taxa", including those provided by Skopin with the label "Homotypus". The validity of these taxa needs a confirmation by reexamination of the type specimens of both "species". In the subsequent key $B$. pterosticha and B. tenuicauda are given in the same couplet.

Distribution. Kazakhstan, Kyrgyzstan, China (Xizang province), Mongolia (LöBL et al. 2008, Ren et al. 2016), Afghanistan (Kaszab 1970)

Regional distribution. North of the Muyunkum Desert, Middle Irtysh region, Balkhash Lake region (SкоріN 1961).

\section{Blaps skopini sp. n.}

(Figs 28-33, 37C)

Type material. Holotype $(\hat{\jmath})$ and paratypes $(1 \hat{\jmath}, 2$ 우 $)$ : 'Southeast Kazakhstan / Ket-

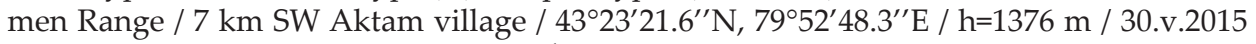
/ leg. A.V. Ivanov' (ZIN); Paratypes: 1 ô, 1 q: 'Southeast Kazakhstan / Sugates Mountains / 4.ix.1968 / leg. N.G. Skopin' (ZIN); 1 §, 3 우: 'Southeast Kazakhstan / Ketmen Range / 4

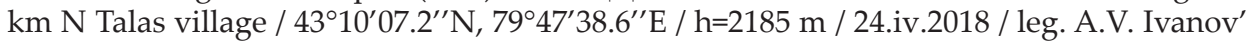
(ZIN); 2 ồ: 'Southeast Kazakhstan / Ketmen Range / 4 km N Talas village / 4310'07.2”'N, 7947'38.6”'E / h=2185 m / 09.iv.2019 / leg. A.V. Ivanov' (IEPaAY, ZIN); 1 ð’: 'Southeast Kazakhstan / 14 km NW Aktogay village / 43¹7'43.9'” N, 7859'27.7' E / h=1006 m / 24.v.2016 / leg. A.V. Ivanov' (ZIN).

Description. Male. Body black, mat, slender. Anterior margin of epistoma weakly emarginate, straight in middle. Lateral margins of epistoma straight. Lateral margins of genae straight in anterior half, rounded at base. Lateral margins of head with indistinct emargination between epistoma and genae. Head widest at level of eyes and temples. Head 1.41 times as wide as interocular distance. Antennomeres 10-11 reaching base of pronotum when directed backwards. Ratio of length/width of antennomeres 2-11 as 6 (8), 
31 (9), 11 (9), 11 (9), 11 (9), 15 (11), 10 (8), 10 (9), 10 (10), 14 (10). Mentum hexagonal, with weak outer angles. Punctation of head sparse, dense (distance between punctures subequal to or smaller than puncture diameter), bottom of punctures with one microgranule.

Pronotum transverse (1.32 times as wide as long), widest at middle, 1.94 times as wide as head. Ratio of pronotal width near anterior angles to widest part and that at base $4.1: 7.8: 6.2$. Disc of pronotum weakly convex, narrow flattened along lateral sides and base. Anterior margin of pronotum widely emarginate, lateral margins widely rounded in anterior third and weakly rounded in posterior half, base of straight. Disk completely beaded except for apical middle. Anterior angles widely rounded, posterior angles narrowly rounded and right. Punctures of pronotum similar to those on head, strongly dense, puncture subcontiguous, sparser in middle (distance between punctures smaller than puncture diameter). Prothoracic hypomera with small wrinkles and covered with sparse small granules. Hypomera along lateral margins narrowly excavate.

Elytra weakly convex, elongate (2.1 times as long as wide together), widest at middle, 3.86 times as long and 1.37 times as wide as pronotum, 2.39 times as wide as head. Caudal extension of elytra (mucro) distinct, $5 \mathrm{~mm}$ long; elytra 4 times as long as mucro. Elytra with rasp-like punctures, punctation at sides becoming denser. Epipleura with fine wrinkles and sparse fine rasp-like punctation. Hair tuft between abdominal ventrites 1 and 2 present. Abdominal ventrites 1-3 with large transverse wrinkles in middle, ventrites 4-5 without wrinkles. Abdominal ventrites 1-3 covered with sparse small granules and
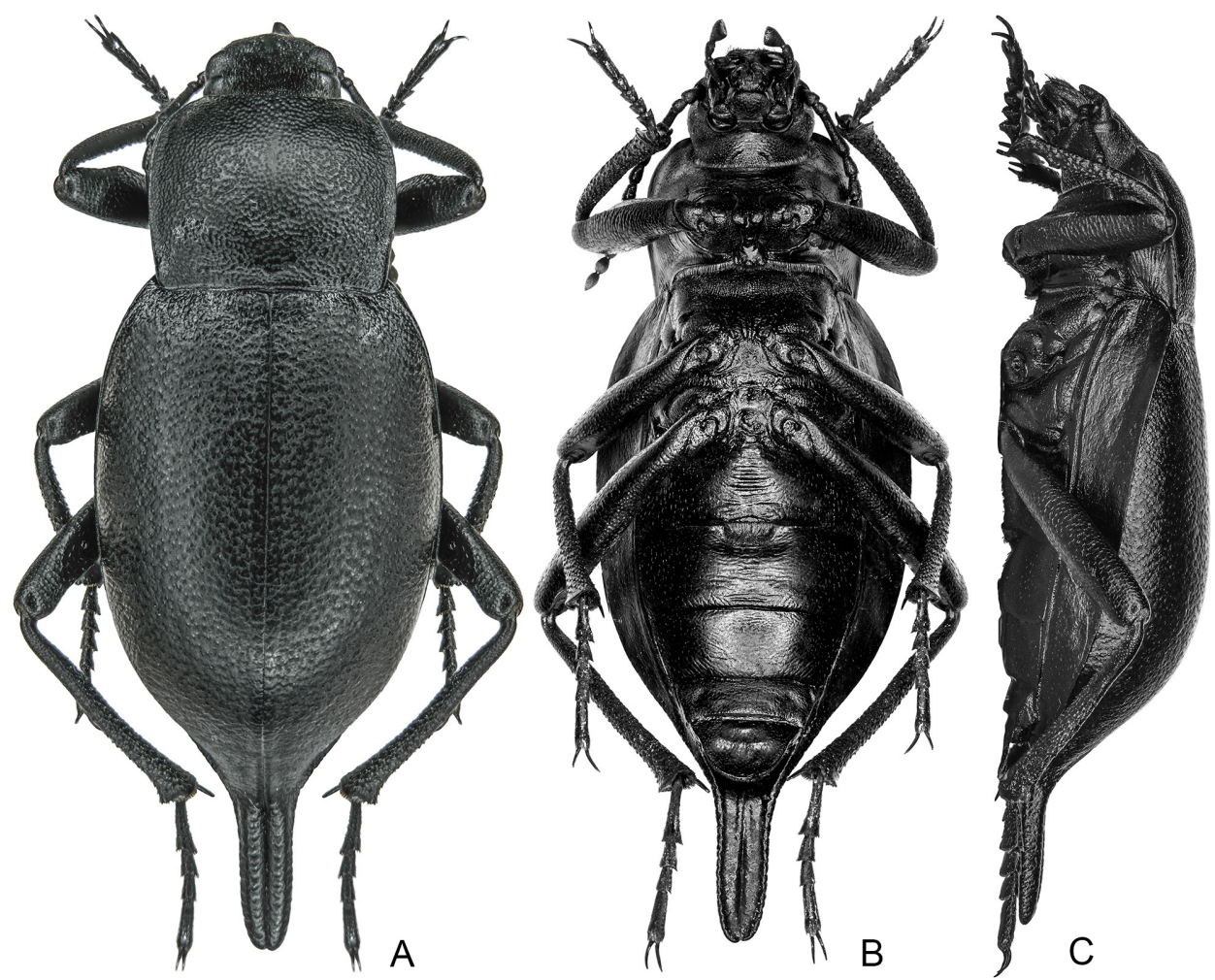

Fig. 28. B. skopini sp. $\mathrm{n}$., habitus, $\widehat{\partial}$. $\mathrm{A}=$ dorsal view; $\mathrm{B}=$ ventral view; $\mathrm{C}=$ lateral view 
rasp-shaped punctures, ventrite 4 with only rasp-like punctures, ventrite 5 with simple punctures, completely beaded except for base.

Legs slender. Ratio of lengths of femora, tibiae and tarsi of fore, middle and hind legs $6.5: 5.5: 3.2 ; 7.2: 6.3: 4.1 ; 9.1: 8.2: 4.7$. All tarsomeres with bifurcated setal brush.

Anterior margin of male inner sternite VIII weakly emarginate, straight in middle, accessory gland of sternite VIII moderately long and thin, middle of sternite without hairs. Rods of spiculum gastrale merged at apex, forming long common stem, lobes of spiculum gastrale elongated. Aedeagus length 4.3-4.4 mm, width 0.8-0.9 mm. Aedeagus weakly Cshaped. Basal third of lateral margins of parameres almost straight, middle widely rounded, apical third strongly acuminate at apex. Parameres length $1.4 \mathrm{~mm}$, width $0.5 \mathrm{~mm}$. Sides of parameres with two longitudinal impressions: one longer beginning from base and another shorter and disposed at middles.

Body length $27-28 \mathrm{~mm}$, width $9.7-9.9 \mathrm{~mm}$.

Female. Shape of body and punctation similar to those of male. Head 1.26 times as wide as interocular distance. Antennomeres 10-11 reaching base of pronotum when directed backwards. Ratio of pronotal width near anterior angles, in widest part and at base $3.8: 6.9: 6.4$. Elytra elongate (1.72 times as long as wide together), 3.16 times as long and 1.38 times as wide as pronotum, 2.7 times as wide as head. Mucro short $(1.6 \mathrm{~mm})$.

Ovipositor moderately long. Apical lobes straight in basal thirds, weakly arcuately rounded in middle, apical third weakly arcuately emarginate. Apex of lobes acute. An-
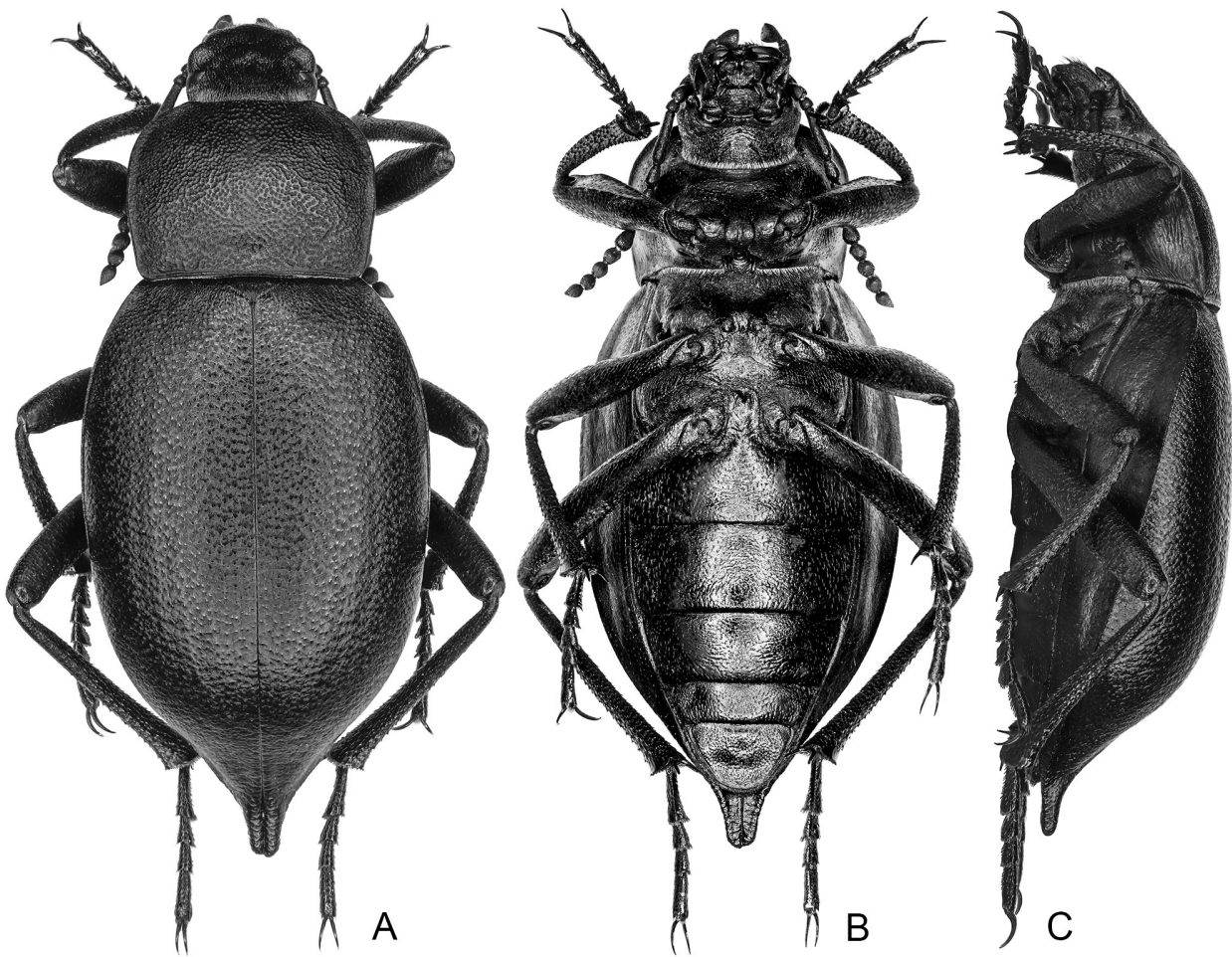

Fig. 29. B. skopini sp. $\mathrm{n}$., habitus, + . $\mathrm{A}=$ dorsal view; $\mathrm{B}=$ ventral view; $\mathrm{C}=$ lateral view 
terior margin of proctiger with deep narrow emarginated in middle. Basal duct of spermatheca between vagina and reservoirs long, gland of spermatheca short. Bases of reservoirs of spermatheca thin, their apical half wider, 1st reservoir twice larger than 2nd. Stem of spiculum ventrale moderately long and thin.

Body length 22-24 mm, width 9-9.2 mm.
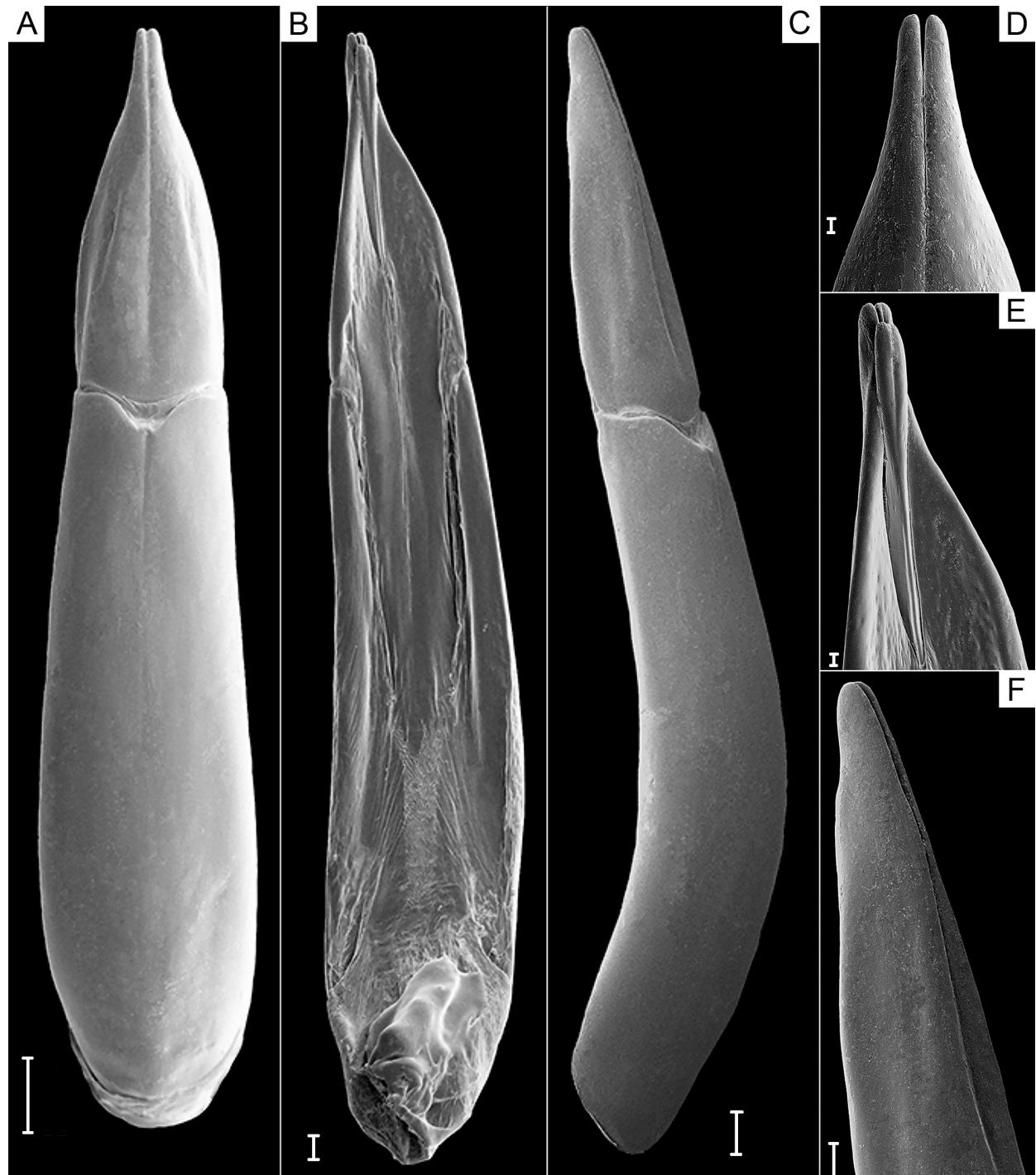

Fig. 30. B. skopini sp. n., aedeagus. $\mathrm{A}=$ dorsal view; $\mathrm{B}=$ ventral view; $\mathrm{C}=$ lateral view; $\mathrm{D}=$ apical piece, dorsal view; $\mathrm{E}=$ the same, ventral view; $\mathrm{F}=$ the same, lateral view. Scale bars: $30 \mu \mathrm{m}$ for $\mathrm{D}, \mathrm{E}, 100 \mu \mathrm{m}$ for $\mathrm{B}, \mathrm{F}, 200 \mu \mathrm{m}$ for $\mathrm{C}, 300 \mu \mathrm{m}$ for $\mathrm{A}$ 
Etymology. The species is named after the late Nikolay Georgievich Skopin famous specialist on Central Asian Tenebrionidae.
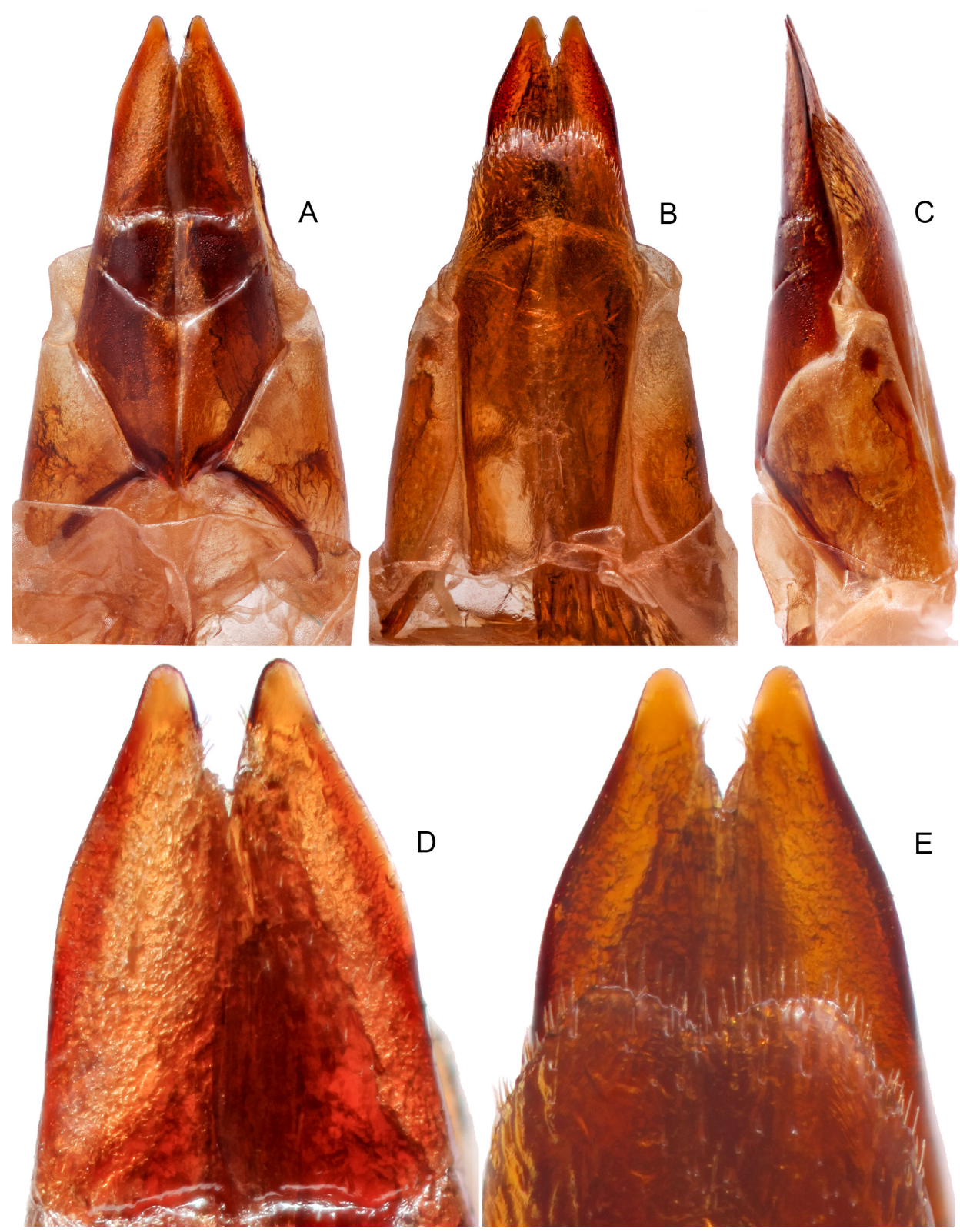

Fig. 31. B. skopini sp. $\mathrm{n}$. A-C =ovipositor: $\mathrm{A}=$ ventral view; $\mathrm{B}=$ dorsal view; $\mathrm{C}=$ lateral view; $\mathrm{D}-\mathrm{E}=$ apical lobes: $\mathrm{D}=$ ventral view, $\mathrm{E}=$ dorsal view 
Differential diagnosis. The body shape and punctation of this new species is similar to B. granulata, B. granulipennis and B. tsharynensis. Blaps skopini sp. n. differs from:

- B. granulata in the more elongate pronotum (pronotum of B. granulata 1.4 times as wide as long); longer mucro of males (male elytra of $B$. granulata 5.5 times as long as mucro); in contrast to those of Blaps skopini sp. n., the sides of parameres of $B$. granulata are smooth, without longitudinal impression, slightly and gradually arched to apex (viewing from above), apex of the latter is straight (Fig. 28B);

- B. granulipennis in the coarser and denser punctation of the pronotum (punctures in Blaps skopini sp. n. are subcontiguous, while in B. granulipennis are subcontiguous everywhere except for middle); longer mucro of males and females (elytra of $B$. granulipennis 7.9 times as long as mucro); sides of parameres of $B$. granulipennis are widely rounded to the apex, with obtuse distinct emargination near the apex; lateral sides of apex of parameres are straight and parallel relative to each other (Fig. 27A);

- $\quad$ B. tsharynensis in the coarser and denser punctation of the pronotum (punctures in Blaps skopini sp. n. are subcontiguous, while they in $B$. tsharynensis are not contiguous); longer mucro of males and females (elytra of B. tsharynensis 10.5 times as long as mucro); larger and more distinct hair tuft between abdominal ventrites 1 and 2 (Figs 35B); parameres of Blaps skopini are elongated (2.48 times as long as wide), while parameres of $B$. tsharynensis are shorter (1.8 times as long as wide); joined apex of parameres in Blaps skopini sp. $\mathbf{n}$. is thin and long (Fig. 30), while

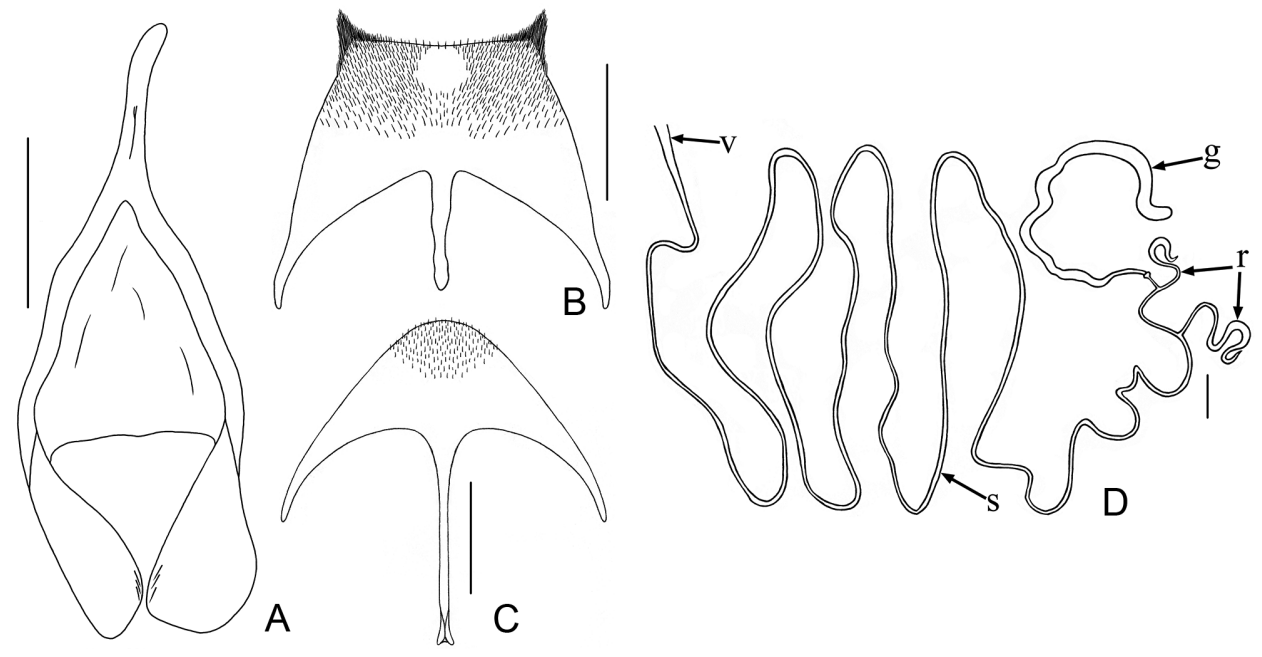

Fig. 32. B. skopini sp. n., details of structure. $\mathrm{A}=$ spiculum gastrale; $\mathrm{B}=$ male inner sternite VIII, $\mathrm{C}=$ spiculum ventrale; $\mathrm{D}=$ female genital tube $(\mathrm{v}=$ vagina, $\mathrm{s}=$ basal duct of spermatheca, $r=$ reservoirs, $g=$ accessory gland of spermatheca). Scale bars $=1 \mathrm{~mm}$ 
that in B. tsharynensis is thin, but short; reservoirs of spermatheca of Blaps skopini sp. n. are thin and fusiform, while reservoirs of spermatheca in $B$. tsharynensis are elliptic; stem of spiculum ventrale of the new species is thinner than that in B. tsharynensis.

\section{Blaps tenuicauda Seidlitz, 1893}

(Fig. 33)

Seidlitz 1893: 307; Skopin, 1960: 56 (larva); Skopin, 1961: 191; Skopin, 1968: 86.

Material examined (ZIN). 1 +, 'South Kazakhstan / South-Kazakhstan Region / Sharapkhana village / 06.v.1964 / leg. N.G. Skopin' [in Cyrillics] [4151'52.60”N N, 69²6’43.72”'E]; 1 ${ }^{\lambda}$, 'South Kazakhstan / South-Kazakhstan Region / Arys city / 14.v.1967 / leg. N.G. Skopin' [in Cyrillics] [42²6'42.32”N $\left.68^{\circ} 51^{\prime} 31.23^{\prime \prime} \mathrm{E}\right]$.

Distribution. Kazakhstan (Löвl et al. 2008), Uzbekistan (Sкоріn 1961).

Regional distribution. South Kazakhstan, hilly steppe between Tashkent and Shymkent cities (Sкоріл 1961); Syrdarya River region (Sкоріл 1968).

Comments. See comments at Blaps pterosticha.
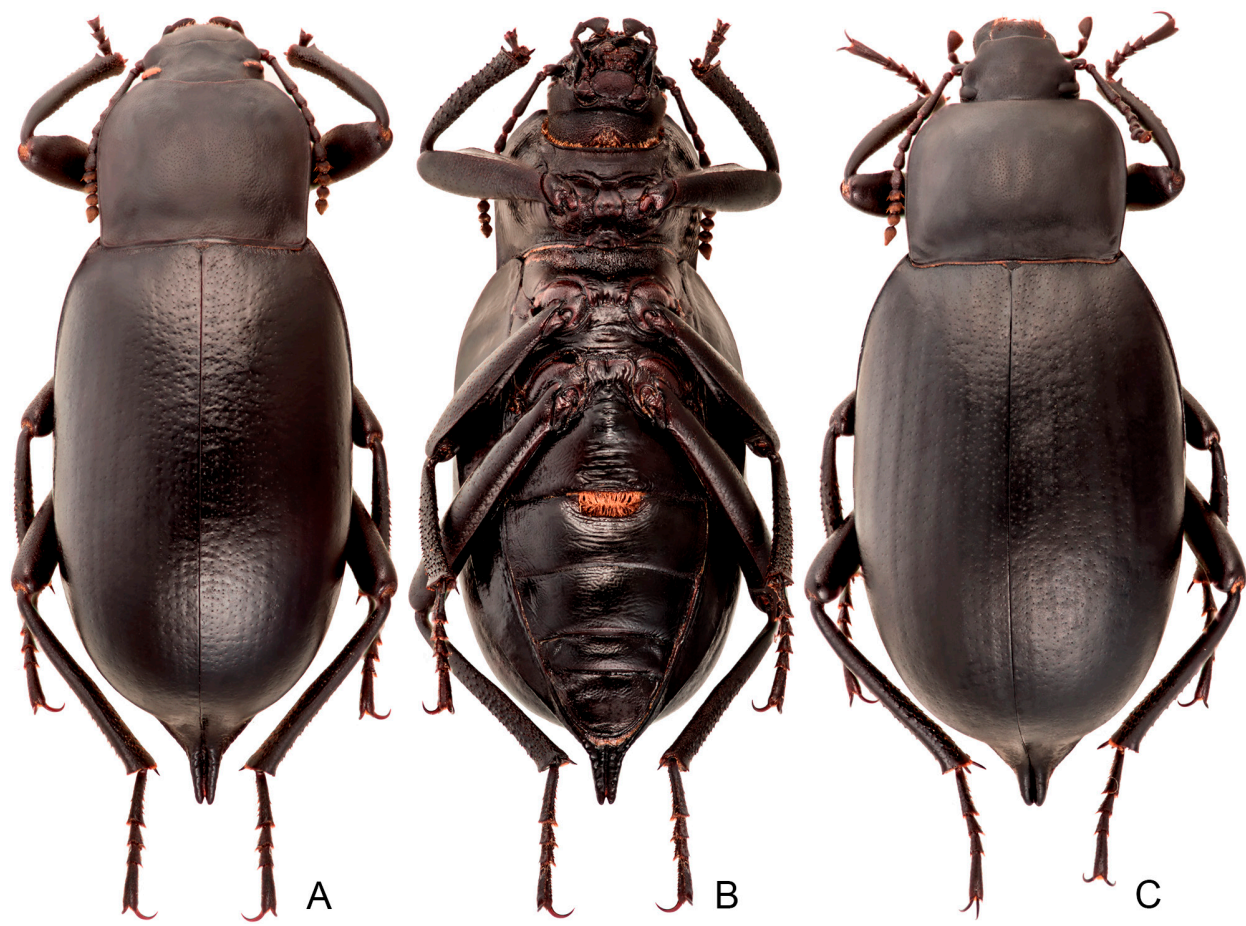

Fig. 33. B. tenuicauda, habitus. $\mathrm{A}, \mathrm{B}=\widehat{\mathrm{O}} ; \mathrm{C}=q$; $\mathrm{A}, \mathrm{C}=$ dorsal view; $\mathrm{B}=$ ventral view 
Blaps transversalis Fischer von Waldheim, 1844

(Fig. 34)

Fischer von Waldheim, 1844: 105; Seidlitz 1893: 285 (“Turkestan”); Skopin, 1960: 55 (larva); Skopin, 1961: 191; Skopin, 1968: 86; Arnoldi \& Medvedev, 1969: 404; Ren et al. 2000; 27; Ren et al., 2016: 171.

Material examined (ZIN). 1 के, 'Southeast Kazakhstan / Alma-Ata Region / canyon of Kopaly River / 19.v.1909 / leg. Nedovpuk[ov]' [in Cyrillics] [44¹'11.62"N, 75 5’54.61"E]; $1 \sigma^{\lambda}$, 'Southeast Kazakhstan / env. of Jambyl / Ulanbel village / 02.v.1960 / leg. L. Serkova'

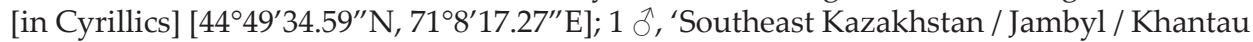

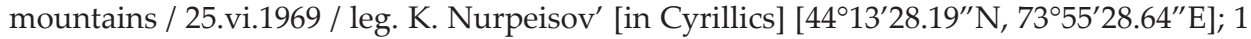
ㅇ, 'Kyrgyzstan / Bishkek city / 15.v.1907 / leg. A. Jakobson' [in Cyrillics] [4253'10.32”N, 74³2'5.29”'E]; $10^{\wedge}$, ‘Kyrgyzstan / Chu River / Boom canyon / 30.iv.1956 / leg. N.G. Skopin'

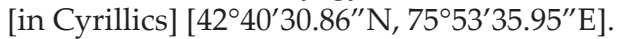

Distribution. Kazakhstan (Arnoldi \& Medvedev 1969), China (Löвl et al. 2008), Kyrgyzstan (SKоріN 1961, 1968).

Regional distribution. Between Talas and the Chu rivers (SKорIN 1961); eastern part of the Karatau Range, the Muyunkum Desert and valley area near the Chu River (SKOpIN 1968).
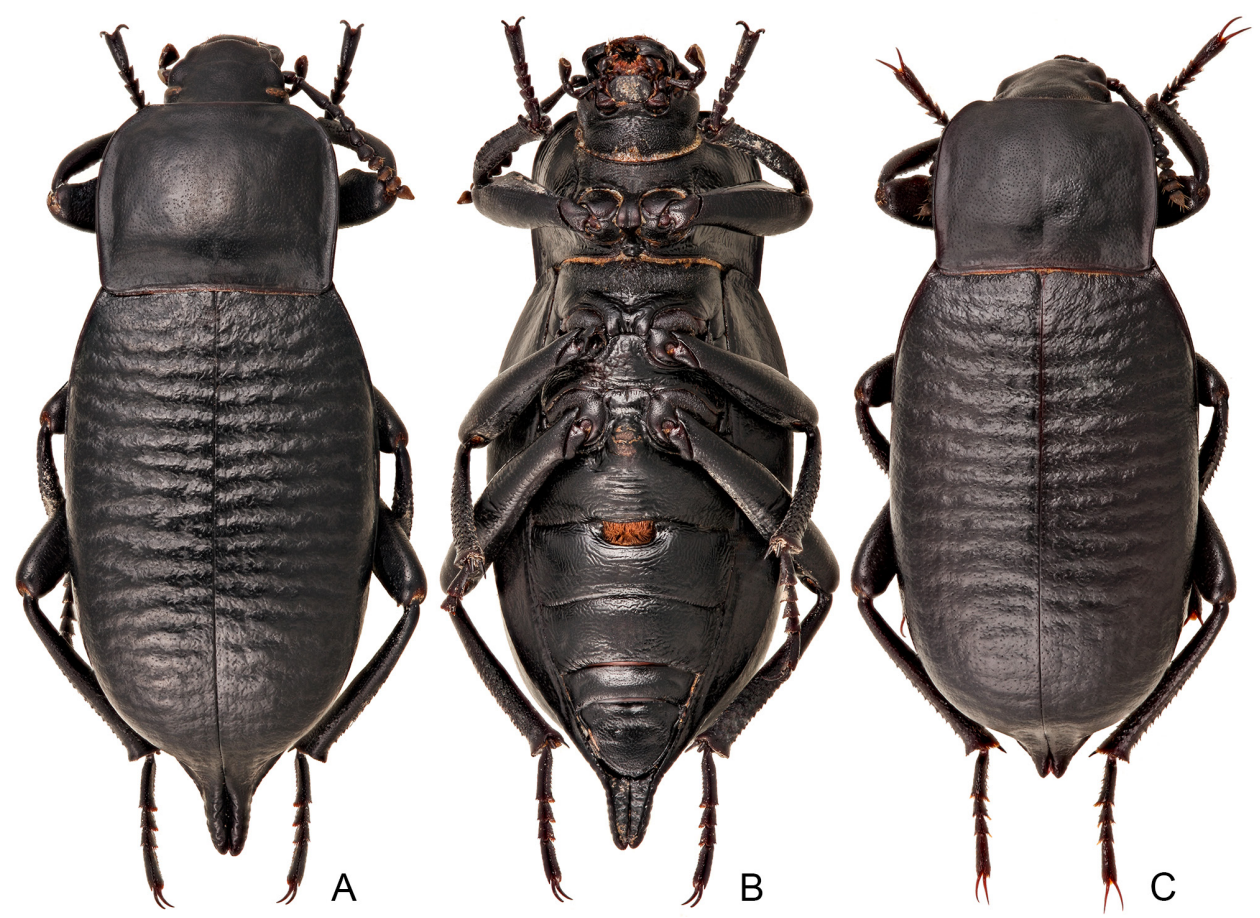

Fig. 34. B. transversalis, habitus. $\mathrm{A}, \mathrm{B}=\mathrm{O} ; \mathrm{C}=+$; $\mathrm{A}, \mathrm{C}=$ dorsal view; $\mathrm{B}=$ ventral view 
Blaps tsharynensis tsharynensis Skopin, 1961

(Figs 35, 36B)

Skopin, 1961: 190; Skopin, 1964: 390; Skopin, 1966: 339.

Type material examined (ZIN). Holotype: ${ }^{\lambda}$, 'Southeast Kazakhstan / Alma-Ata Region

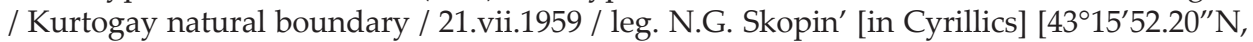
78 58'25.09”'E]. Allotype: + , 'Southeast Kazakhstan / Alma-Ata Region / Kurtogay natural

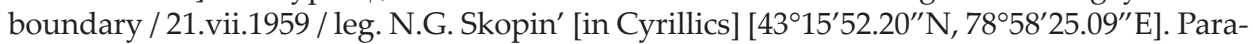
types: 1 $\lambda$, 'Southeast Kazakhstan / Alma-Ata Region / middle course of Charyn River / 23.iv.1960 / leg. N.G. Skopin' [in Cyrillics]; 1 đ̃, 'Southeast Kazakhstan / Alma-Ata Region / Kurtogay natural boundary / 12.v.1961 / leg. N.G. Skopin' [in Cyrillics] [43¹5’52.20”N, 78 58'25.09" E]; 1 ㅇ, 'Southeast Kazakhstan / Alma-Ata Region / Kurtogay natural boundary / 05-19.vii.1959 / leg. N.G. Skopin' [in Cyrillics] [43¹5’52.20”N, 7858’25.09”E].

Material examined (ZIN). 1 o, 1 क ,'Southeast Kazakhstan / Alma-Ata Region / Lavar

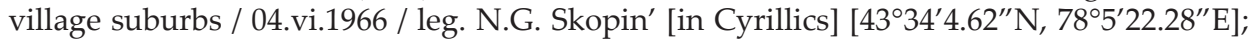
2 후, 2 우우, 'Southeast Kazakhstan / Alma-Ata Region / 40 km south-west of Dzharkent

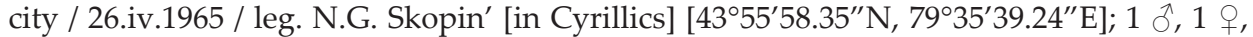
'Southeast Kazakhstan / Alma-Ata Region / 60 km west of Gulshat village / 17.ix.1964 / leg. N.G. Skopin' [in Cyrillics] [46 36'57.83”N, 73³4'7.28”'E]; 1 ô, 1 ㅇ, 'Southeast Kazakhstan /

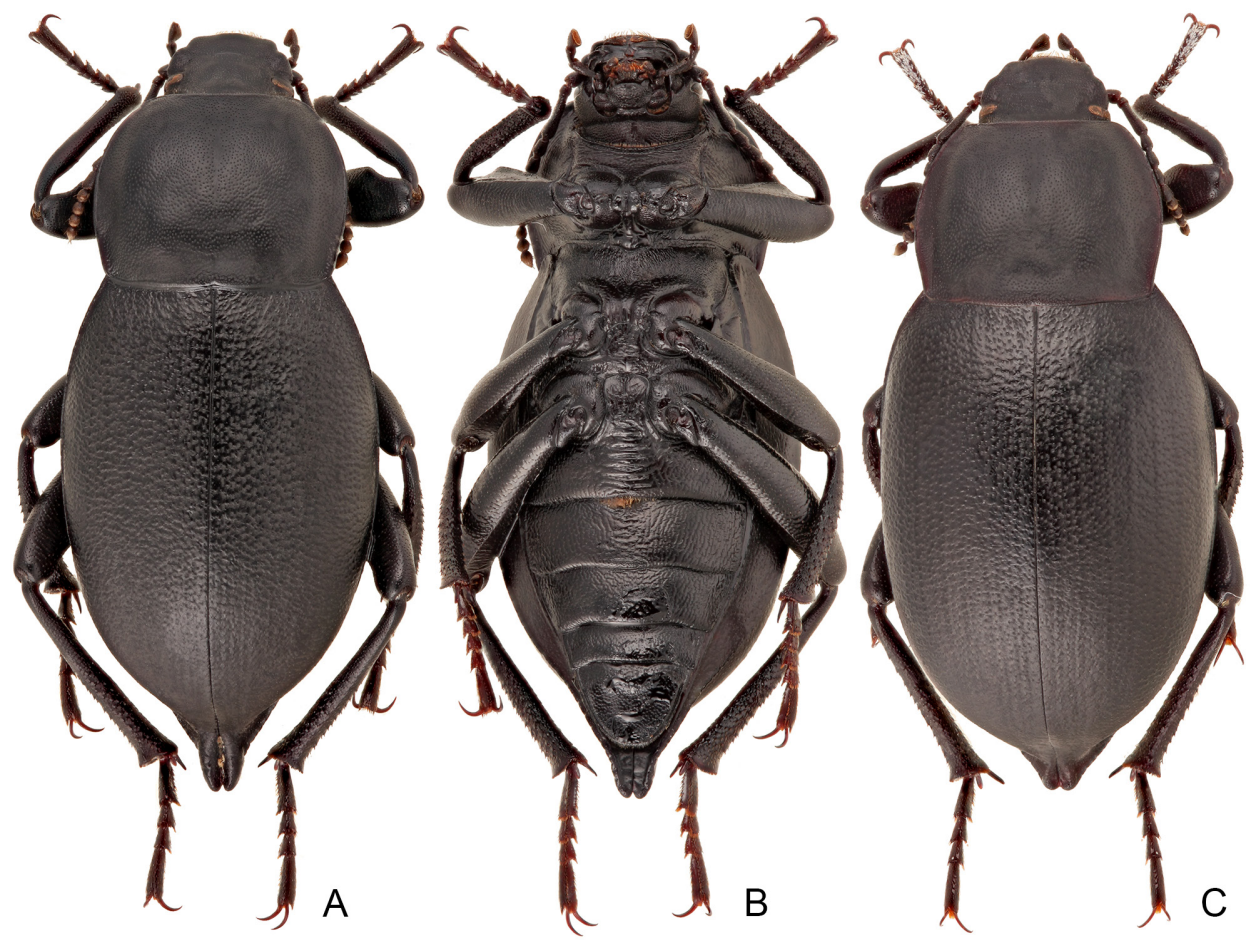

Fig. 35. B. tsharynensis tsharynensis, habitus. $\mathrm{A}, \mathrm{B}=\mathrm{O} ; \mathrm{C}=q ; \mathrm{A}, \mathrm{C}=$ dorsal view; $\mathrm{B}=$ ventral view 


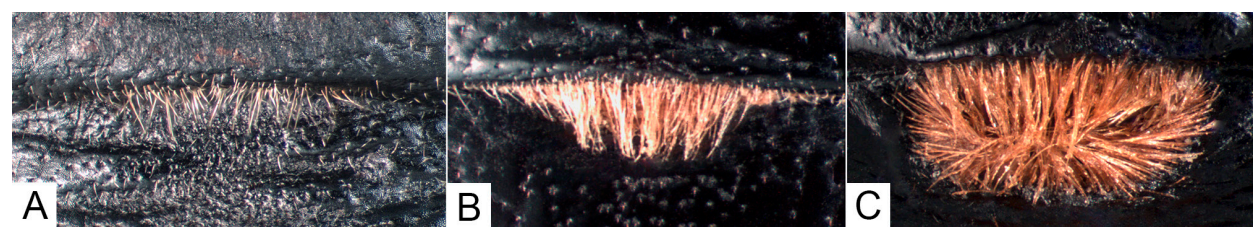

Fig. 36. Hair tuft between 1 st and 2nd abdominal ventrites. $\mathrm{A}=B$. motschulskiana, $\mathrm{B}=\mathrm{B}$. tsharynensis tsharynensis, $\mathrm{C}=$ B. skopini sp. $\mathrm{n}$.

Alma-Ata Region / lower reaches of Karatal River / 28.v.1965 / leg. N.G. Skopin' [in Cyrillics] [46ㅇ' $\left.18.41^{\prime \prime} \mathrm{N}, 77^{\circ} 9^{\prime} 44.45^{\prime \prime} \mathrm{E}\right]$.

Distribution. Kazakhstan (LöвL et al. 2008).

Regional distribution. The Kurtogay canyon, Ili River region (near the spring of Ayak-Kalkan) (Sкорім 1961).

Blaps tsharynensis balchashensis Skopin, 1966

(Fig. 37)

Skopin, 1966: 338.

Type material examined (ZIN). Holotype: 0 , ‘Kazakhstan / North of Balkhash Lake / 60 км W Gulshat village/ 17.ix.1964 / leg. N.G. Skopin' [in Cyrillics]. Paratypes: 1 d, 1 , , 'Central Kazakhstan / Karaganda Region / 40 km south of Sayak village / 21.ix.1964 / leg.

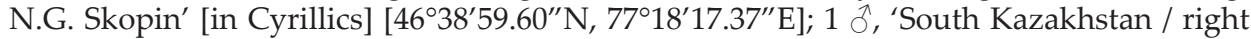
bank of Chu River / 13.ix.1964 / leg. N. G. Skopin' [in Cyrillics].

Material examined (ZIN). 5 ', 'Southeast Kazakhstan / Alma-Ata Region / lower reaches of Karatal River / 28.v.1965 / leg. N.G. Skopin' [in Cyrillics] [46 ${ }^{\circ} 18.41^{\prime \prime N}$, 7709'44.45"E]; 1 + , 'Southeast Kazakhstan / Alma-Ata Region / 30 km west of Gulshat village / 17.ix.1964 / leg. N.G. Skopin' [in Cyrillics] [46³6’59.25”N, 7356’35.18”E].

Distribution. Central and Southeast Kazakhstan (Balkhash Lake region) (Sкорім 1966).

\section{Blaps turcomanorum Seidlitz, 1893}

(Figs 27F, 38)

Seidlitz, 1893: 305

Material examined (ZIN). 1 ๙ , 1, 'Kazakhstan / Dzhambul [Jambyl] // Blaps turcomanorum'.

Distribution. Kazakhstan (first record for the country), Uzbekistan (LöвL et al. 2008).

Regional distribution. Southeast Kazakhstan. 

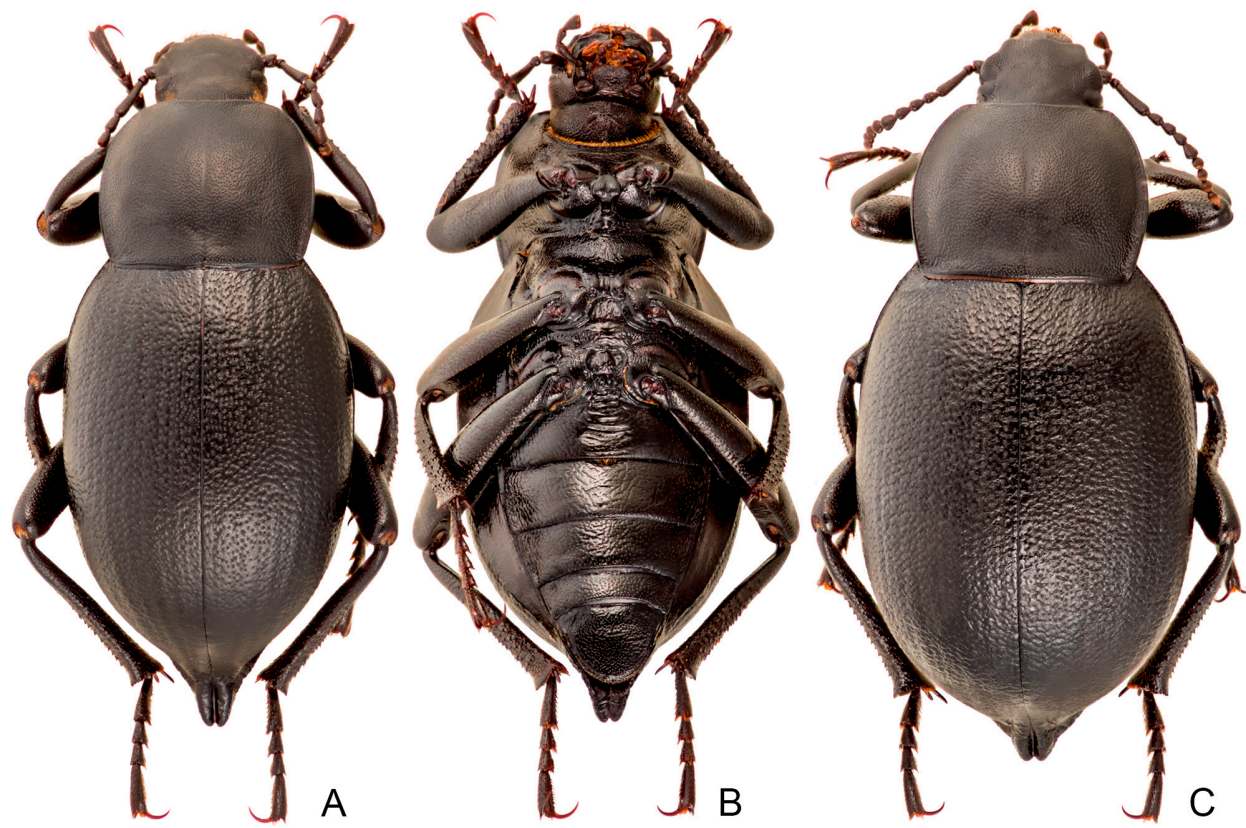

Fig. 37. B. tsharynensis balchashensis, habitus. $A, B={ }^{\lambda} ; C=$; $A, C=$ dorsal view; $B=$ ventral view
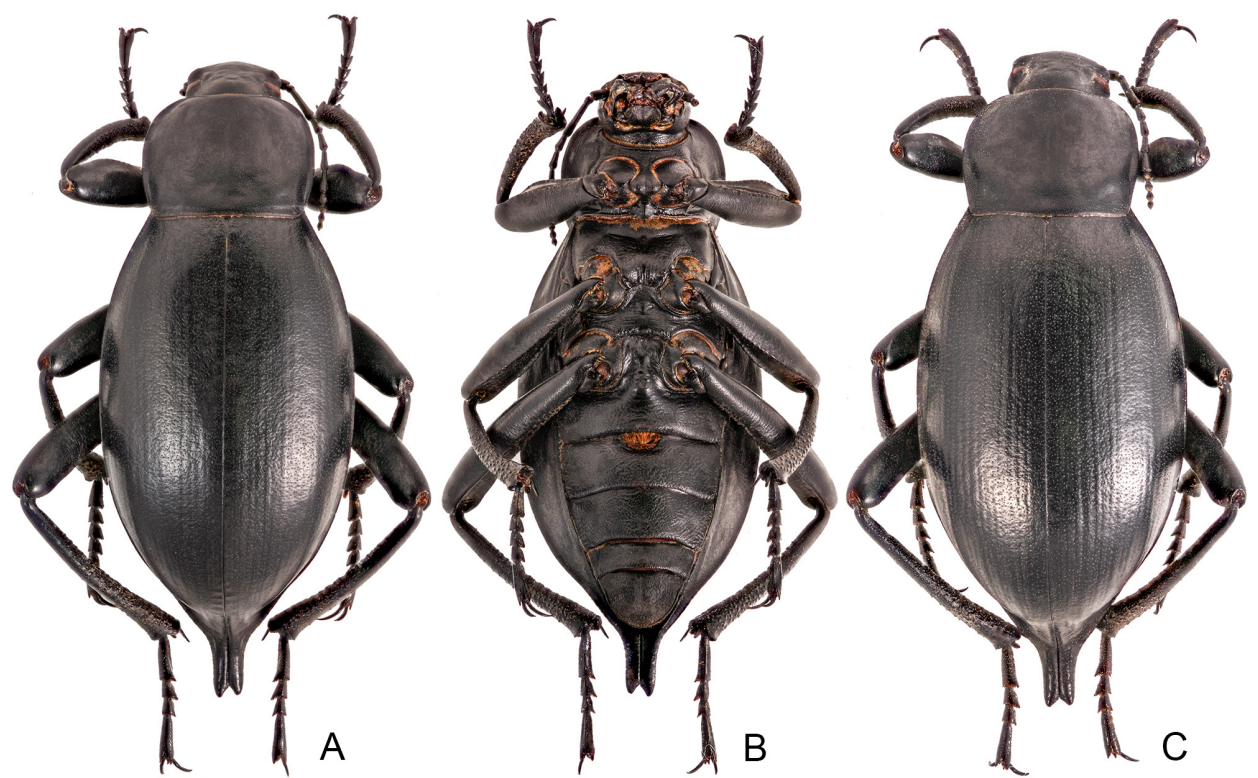

Fig. 38. $\mathrm{B}$. turcomanorum, habitus. $\mathrm{A}, \mathrm{B}=\mathrm{d} ; \mathrm{C}=$ + $; \mathrm{A}, \mathrm{C}=$ dorsal view; $\mathrm{B}=$ ventral view 
Blaps virgo Seidlitz, 1893

(Fig. 39)

Seidlitz, 1893: 307; Skopin, 1961: 190; Ren et al. 2000; 27; Ren et al., 2016: 178.

Material examined. NHM. 1 ふै, 'Haberhauer / Turkestan / 89 // Blaps akinina All // sagitta // Cotypus / Blaps sagitta Seidl. / N. Skopin design. / 1978'. ZIN. 1 ô, 4 우오 ‘Naryn mountains / Semirechye Region / 6.vi.1905. / leg. Nezhivov' [in Cyrillics]; $1 \hat{\delta}$, 'Naryn mountains / Semirechye Region / 8.vi.1905. / leg. Nezhivov' [in Cyrillics]; 2 우, 'Naryn mountains / Semirechye Region / 11.vi.1905. / leg. Nezhivov' [in Cyrillics]; 1 ô, Przhevalsk [Karakol] / 28.vi.04 / leg. Nezhivov' [in Cyrillics]; 2 우, 'Naryn mountains / 9.vii.1904 / leg. Nezhivov' [in Cyrillics]; 3 우, ‘Naryn town / 10.vii.1904 // leg. Herz'; 1 ㅇ, 'Naryn town / 11.vii.1904'; 1 ㅇ, ‘Naryn town / 20.vi.04 / leg. Nezhivov' [in Cyrillics]; $3 \hat{\jmath} \delta^{\lambda}, 1$ + , 'Southeast Kazakhstan / Dzungarian Alatau / 1.vi.1968 / leg. N.G. Skopin' [in Cyrillics]; 1 ô, 1 +, ‘Dzungarian Alatau / 5 km N Bien River / 22.v.1968 / leg. N.G. Skopin’ [in Cyrillics].

Comments. A population of $B$. virgo with short mucro $(1-1,5 \mathrm{~mm})$ occurs near Bien River (Alma-Ata Region).
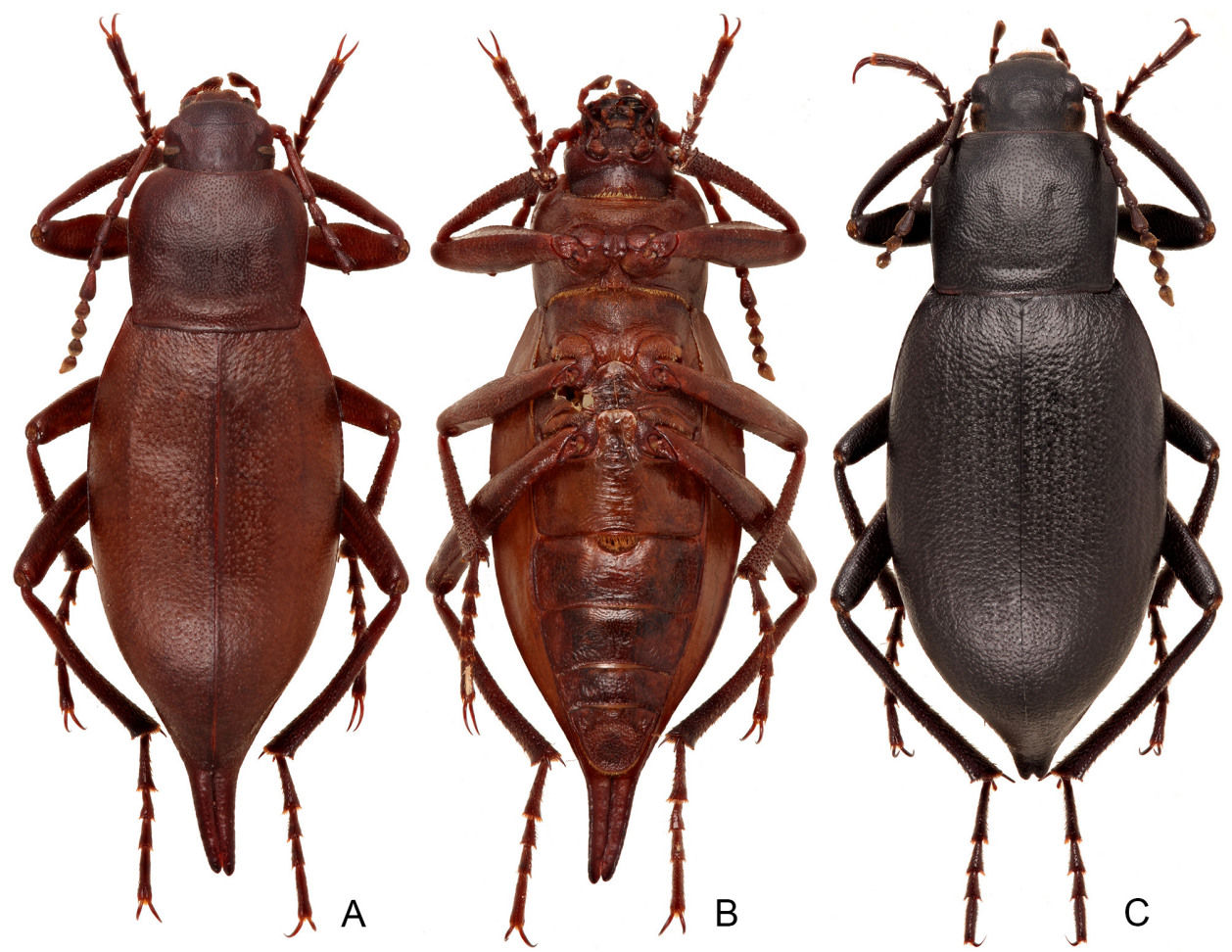

Fig. 39. B. virgo, habitus. $A, B=\widehat{O} ; C=q ; A, C=$ dorsal view; $B=$ ventral view 
Distribution. Kazakhstan, Kyrgyzstan, China (Xinjiang Region) (Löвl et al. 2008, Ren et al. 2016).

Regional distribution. Northern slopes of Terskey Alatau Range (Narynkol Region).

\section{Subgenus Dineria Motschulsky, 1860}

Type species: Blaps confusa Ménétriés, 1832 (= Blaps halophila Fischer von Waldheim, 1820) by the subsequent designation (Medvedev \& Iwan 2006).

\section{Blaps halophila Fischer von Waldheim, 1820}

Fischer von Waldheim, 1820: tab. xvi, fig. 7; Fischer von Waldheim, 1822: 190; Ménétriés, 1832; 201 ("Blaps confusa"); Skopin, 1960: 51 (larva); Arnoldi \& Medvedev, 1969: 403;

Chigray et al. 2016: 12. (see figures in: Chigray et al. 2016: figs 12D-F)

Type material examined (ZIN). Holotype of Blaps convexicollis Motschulsky, 1845: §, 'Mt. Altai // Blaps convexicollis m. Altai'. Lectotype of Blaps confusa Ménétriés, 1832 designated here: $\hat{\delta}$, 'Caucas. // confusa Ménét. Caucas. // Blaps longicollis // Lectotypus / Blaps confusa Ménétriés, 1832 / des. I.A. Chigray'.

Material examined (ZIN). 2 우, ‘Kazakhstan / Zaisan district / 5.vii.[19]26 / leg. Filatova' [in Cyrillics]; 1 , 'Kazakhstan / Zaisan / Neygy Kuma / into Achnatherum sp. / 10.vi.1958 / leg. N.G. Skopin' [in Cyrillics]; 1 q, ‘Kazakhstan / Zaisan hollow / 10.vi.1958 / leg. N.G. Skopin'; 1 + , 'Semirechye Region / Alatau / Kurdayskiy pass / 16.vii.1906 / leg. N. Radkevich' [in Cyrillics].

Distribution. Part of Central, Southern and Eastern Europe, the European part of Russia, the Great Caucasus, Southern Siberia, North Kazakhstan (Arnoldi \& Medvedev 1969, Abdurakhmanov \& Nabozhenko 2011).

Regional distribution. Karaganda Region (SKOPIN 1960).

\section{Subgenus Prosoblapsia Skopin \& Kaszab, 1978}

Type species Blaps allardiana Reitter, 1889 by the original designation (SKорIN \& KASZAв 1978).

Comments. Medvedev (2001) interpreted the genus Ablapsis Reitter, 1887 as a subgenus of Blaps and some species of Prosoblapsia (Blaps (Ablapsis) compressipes Reitter, 1887, B. (A.) berezowskii G. S. Medvedev, 1998, B. (A.) lucidula G. S. Medvedev, 1998, B. (A.) znoikoi Semenov \& Bogatchev, 1936 and B. (A.) gentilis gentilis Fairmaire, 1887) were included by him in the subgenus Ablapsis. However, further specialists have not accepted this opinion (LöвL et al., 2008, ReN et al., 2016). The taxonomic position and composition of Ablapsis and Prosoblapsia will be discussed in further works. 
Blaps transversimsulcata transversimsulcata Ballion, 1878

(Fig. 40)

Ballion, 1878: 301; Seidlitz, 1893: 296 (“Turkestan”); Skopin, 1960: 52 (larva); Skopin, 1961: 190; Skopin, 1968: 84; Skopin, 1977: 149; Ren et al., 2016: 196.

Material examined (ZIN). 1 đ̊, 1ㅇ, ‘Tian Shan, Musart // coll. N. Skopin' [in Cyrillics]; 1 ठ̊, 'Ost-Turkestan / Acsu River / 1067 m. / v.1903 / leg. Hauser // coll. N. Skopin' [in Cyrillics]; 1 ठิ, 1ㅇ, 'Uzbekistan / Tian Shan / Przhevalsk [Karakol] / 3300 m. / vi.77' [in Cyrillics]; $1 \hat{\delta}$, 'Cat River [tributary of Ili River]/ Dzungarian Region/ leg. Regel'; $1 \hat{\delta}, 1$, 'Env. of Kuldzha / 22.iv.79 / leg. A. Regel // Topotype // Homotype Blaps rudesculpta / det. N. Skopin'.

Comments. Blaps transversimsulcata rudesculpta Semenov Tian-Shansky et Bogatchev, 1936 is a younger synonym of B. t. transversimsulcata (SкоріN 1977), not of Blaps maeander Kraatz, 1885 (mistake in LöвL et al. 2008).

Distribution. Kazakhstan, China (Xinjiang Province) (LöвL et al. 2008, REN et al. 2016).

Regional distribution. Talas Ala-Too and Ugam Ranges (SKopIn 1968).
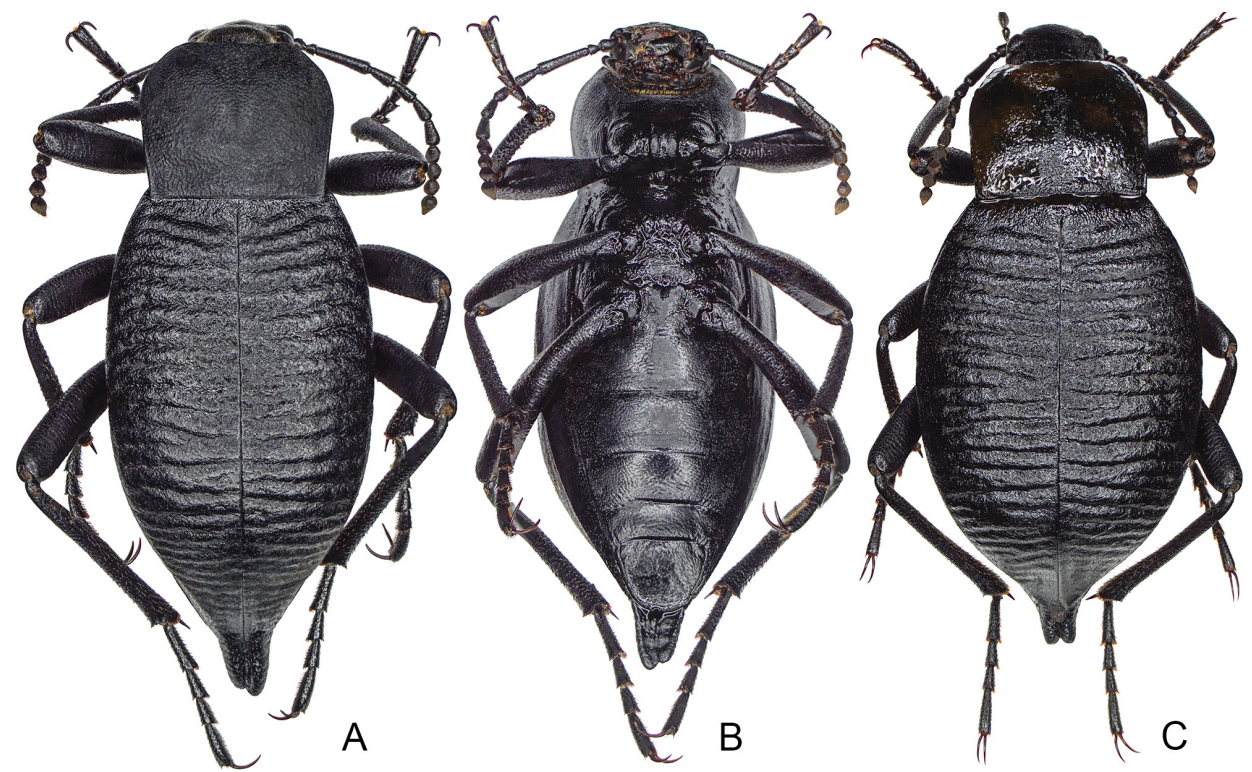

Fig. 40. B. transversimsulcata transversimsulcata, habitus. $A, B=\widehat{O} ; C=q ; A, C=$ dorsal view; $\mathrm{B}=$ ventral view 
Blaps transversimsulcata meandroides Skopin, 1977

(Fig. 41)

Skopin, 1977: 151.

Type material examined (ZIN). Holotype: $\widehat{\jmath}$, 'Southeast Kazakhstan / Alma-Ata Region / Alma-Ata city suburbs / 19.vi.1957 / leg. N.G. Skopin' [in Cyrillics] [43¹2'12.60”N , 7650'37.26"E]. Paratypes: 1 o', 'Southeast Kazakhstan / Alma-Ata Region / Alma-Ata city /

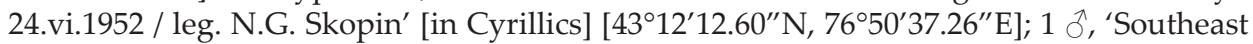
Kazakhstan / Alma-Ata Region / Zailiyskiy Alatau / Khargaly canyon / 12.vii.1969 / leg.

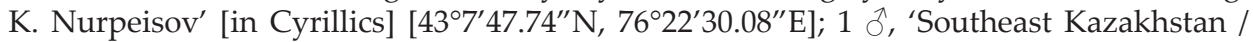
Alma-Ata Region / Alma-Ata city suburbs / 13.vii.1953 / leg. N.G. Skopin' [in Cyrillics]

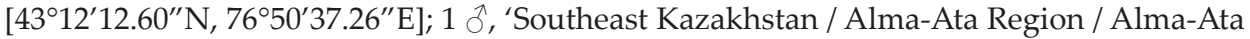

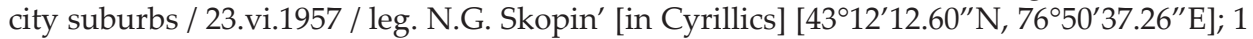
ô, ‘Kazakhstan / Alma-Ata / h = 1400 m / 31.v.1950 / leg. N.G. Skopin'; 1 đ’, ‘o, ‘Kyrgyzstan / Bishkek city / 2.vi.1976 / leg.Yanushev' [in Cyrillics].

Material examined (ZIN). 1 ô, 6 우, 'Eastern Kazakhstan / Alma-Ata Region / Zailiyskiy Alatau Range / gorge of Uzun-Kargaly River [N4344ㅇ.23”, E76²4'9.25”] / 31.05-08. vi.1907 / leg. A. Jakobson' [in Cyrillics].

Distribution. Northern, North-East and Central Tian Shan (Kazakhstan, Kyrgyzstan) (SKOpIN 1977).

Regional distribution. Southeast Kazakhstan (SкорIN 1977).
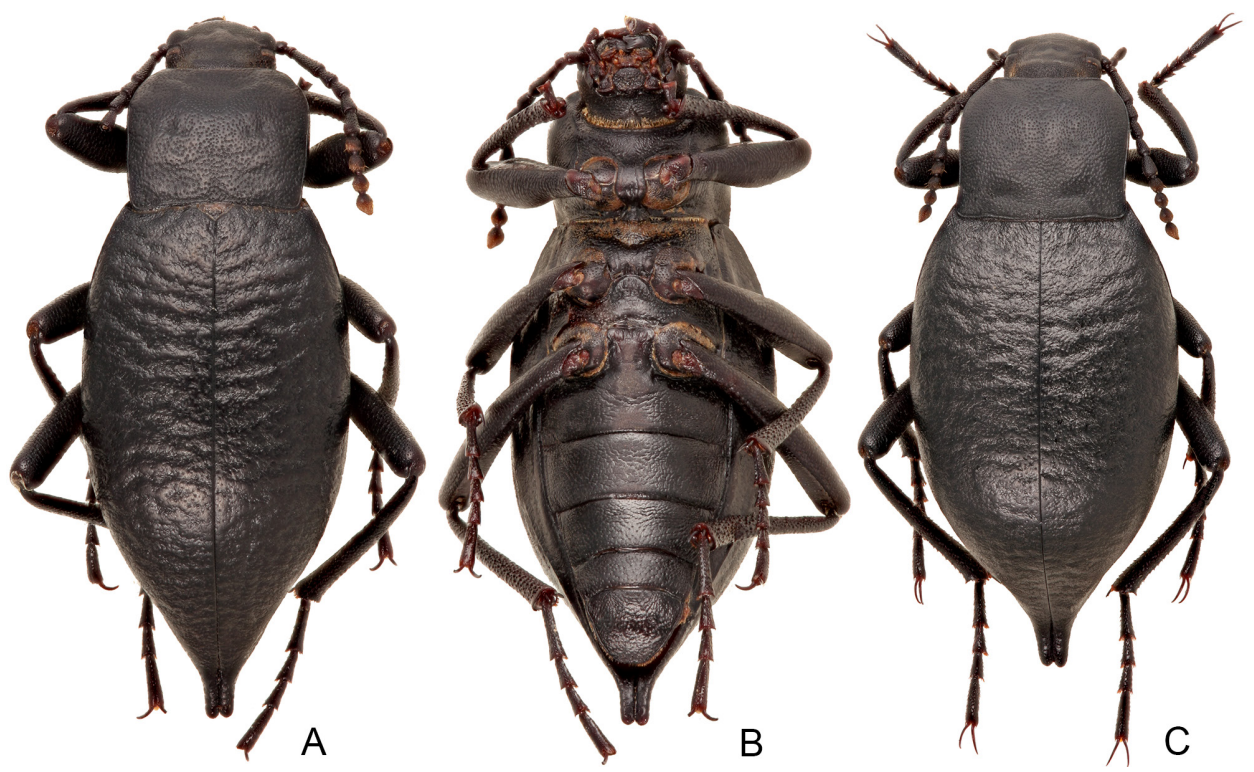

Fig. 41. B. transversimsulcata meandroides, habitus. $\mathrm{A}, \mathrm{B}=\hat{\mathrm{A}} ; \mathrm{C}=$ + $; \mathrm{A}, \mathrm{C}=$ dorsal view; $\mathrm{B}=$ ventral view 


\section{KEY TO THE BLAPS SPECIES \\ OF CENTRAL AND SOUTH KAZAKHSTAN}

1. Ventral lamella between tarsal claws triangular, acute or narrowly rounded at apex. Dorsal margin of metafemora on inner side with small teeth

- Ventral lamella between tarsal claws widely rounded or truncate. Dorsal margin of metafemora without small teeth on inner side

2. Mucro of male and female very short $(0.5-0.7 \mathrm{~mm})$, abruptly bifurcated at base in shape of two triangular processes

B. pruinosa

- Mucro of male and female long (more than $3.0 \mathrm{~mm}$ ), spatulate (not bifurcate)

3. Male without hair tuft between abdominal ventrites 1 and 2 or with group of long sparse hairs (Fig. 36A)

B. motschulskiana.

- Male with hair tuft between abdominal ventrites 1 and 2

4. Mucro of male and female wide, flattened dorsovenrally. Male abdominal ventrite 1 with transverse tubercle. Male aedeagus very long $(9 \mathrm{~mm})$, lateral sides of parameres widely emarginate at middle, apex of parameres rounded (Fig. 27E)

B. faustii

- Mucro of male and female narrow, not flattened. Male abdominal ventrite 1 with rounded tubercle. Male aedeagus shorter $(5-6 \mathrm{~mm})$, lateral sides of parameres straight, apex of parameres acute (Fig. 27F)

B. turcomanorum

5. Male without hair tuft between abdominal ventrites 1 and $2 \quad 6$

- Male with hair tuft between abdominal ventrites 1 and 2

6. Elytra with deep transverse wrinkles

- Elytra not wrinkled or with fine microwrinkles formed by punctures

7. Parameres very narrow and elongate (2.78 times as long as wide), separated by suture only in apical half. Lateral margins of parameres widely rounded along entire length

B. ballioni

- $\quad$ Parameres wider (2.26 times as long as wide), completely separated by suture. Lateral margins of parameres widely rounded in basal half and weakly emarginate near apex 
8. Wrinkles of elytra very deep, straight, subparallel

B. transversimsulcata transversimsulcata

- Wrinkles of elytra shallower and more obliterated, irregular, not subparallel

B. transversimsulcata meandroides

9. Male metatibiae thickened in the middle of inner side or slightly dislodged toward apex

- Male metatibiae without thickening in the middle of inner side or with extension from the middle toward apex

10. Elytra coarsely granulate

B. inflexa

- Elytra punctate

B. halophila

11. Male antennomeres $10-11$ reaching basal quarter of pronotum, but not reaching base of pronotum. Male and female mucro well developed

B. kadyrbekovi

- Male antennomeres 10-11 reaching base of pronotum or basal quarter of elytra. Male and female mucro absent

12. Anterior angles of pronotum widely rounded to obtuse. Pronotal punctures small, not merged with each other

B. caraboides caraboides

Anterior angles of pronotum narrowly rounded, sharp. Pronotal punctures large, merged with each other

B. caraboides intermittens

13. Wrinkles of elytra very deep and transverse

B. transversalis

- Elytra not wrinkled or with very small wrinkles 14

14. Male abdominal ventrite 1 always with tubercle

- Male abdominal ventrite 1 without tubercle or with small tubercle (when body small)

15. Body robust, very wide. Tubercle of male abdominal ventrite 1 transverse or dentate, acute at apex

- Body more elongate. Tubercle of male abdominal ventrite 1 rounded and not acute at apex

16. Body very wide (2.1 times as long as wide). Anterior margin of pronotum deeply emarginate, anterior angles very protruding to head. Spurs of meso- and metatibiae flattened, triangular, acute at apex, asymmetric (Figs 21C,D)

B. holconota 
- $\quad$ Body less wide (2.3 times as long as wide). Anterior margin of pronotum widely emarginate, anterior angles shortly projecting to head. Spurs of meso- and metatibiae flattened, but obtuse and wide at apex, symmetric (Figs 21A,B)

B. deplanata

17. Pronotum subparallel-sided along middle. Apex of parameres acute, lateral sides of parameres with longitudinal impressions along midlength (Fig. 27D)

B. evanida

- Pronotum weakly rounded along middle. Apex of parameres wide, lateral sides of apex of parameres straight, without impressions (Fig. 27C)

B. pterosticha, B. tenuicauda

18. Antennomeres 10-11 elongate (longer than wide). Lateral sides of pronotum not excavate. Body very slender, narrow. Male mucro very narrow and long

B. virgo

- Antennomeres 10-11 rounded or transverse (wider than long). Lateral sides of pronotum always excavate. Body wider. Male mucro short (except for B. skopini sp. n.)

19. Punctation of pronotum fine and sparse (distance between punctures about twice as great as puncture diameter)

- $\quad$ Punctation of pronotum coarse and dense (distance between punctures smaller than puncture diameter), punctures sometimes merged

20. Bead of lateral margins of pronotum coarse or simple. Tubercle of male abdominal ventrite 1 short and wrinkled

- Bead of lateral margins of pronotum simple or thin. Male abdominal ventrite 1 without tubercle

21. Spurs on meso- and metatibiae simple, not flattened and not long. Apices of reservoirs of spermatheca ellipsoidal, 1st reservoir much larger than 2nd, bases of reservoirs distantly separated

B. nitida, B. lethifera

- Spurs on meso- and metatibiae flattened and long. Apices of reservoirs of spermatheca spherical, bases of reservoirs subequal in size, close to each other or with common duct

22. Male metatibiae simple, gradually expanding from base to apex

B. parvicollis parvicollis

- Male metatibiae with thickening only in apical half

B. parvicollis quadricollis 
23. Body slender. Pronotum square. Elytra flattened along suture. Legs long. Male hair tuft between 1st and 2nd abdominal ventrites large and distinct. Accesory gland of spermatheca short (basal duct of spermatheca 6 times as long as the gland)

B. fouquei sp. $\mathbf{n}$.

- Body wider. Pronotum transverse. Elytra weakly convex along suture. Legs shorter. Male hair tuft between 1st and 2nd abdominal ventrites very small and indistinct. Accesory gland of spermatheca long (basal duct of spermatheca twice as long as the gland)

24. Disk of pronotum strongly convex. Pronotal sides steeply sloping and widely explanate along margin

B. tsharynensis tsharynensis

- Disk of pronotum moderately convex. Pronotal sides gently sloping and $\begin{array}{ll}\text { narrowly explanate along margin } & \text { B. tsharynensis balchashensis }\end{array}$

25. Lateral sides of parameres widely rounded, apices straight B. granulata

- Lateral sides of parameres with emarginate outline before apex 26

26. Pronotal punctation dense, punctures merged everywhere except for middle. Male mucro short, female mucro slightly expressed. Lateral margins of parameres widely rounded at apex, with emargination near apex; lateral outline of apex of parameres straight and subparallel-sided

B. granulipennis

- Pronotal punctation extremely dense, punctures merged over entire surface. Male mucro long, female mucro well expressed. Basal third of lateral margins of parameres straight, middle widely rounded; lateral outline of apex of parameres straight, but not subparallel-sided $\quad$ B. skopini sp. $\mathbf{n}$.

Acknowledgements - The authors are much obliged to V. Yu. Shmatko (Institute of Arid Zones RAS, Rostov-on-Don, Russia) for the preparation of photographs of some species, to A. M. Shapovalov (St. Petersburg, Russia) for providing the material from Kazakhstan, to A. G. Kirejtshuk (ZIN, St. Petersburg, Russia) and M. V. Nabozhenko (Precaspian Institute of Biological Resources of Dagestan Federal Researche Centre of the Russian Academy of Science, Makhachkala, Russia) for valuable comments and corrections during preparation of the manuscript.

The authors are also cordially thank the staff of the laboratory of insect taxonomy of ZIN RAS: M. G. Volkovitsh, S. V. Andreeva, A. V. Kovalev and A. G. Moseyko for various assistance and support in studies, to Purchart Luboš (Mendel University in Brno, Brno, Czech Republic) and Lukáš Sekerka (NMP) for assistance in studies of Tenebrionidae in the Natural History Museum Prague, to Harald Schilhammer (NHM) for provided material.

The studies were carried out under the framework of the Russian State Research Project No. AAAA-A19-119020690101-6 and the Russian Foundation for Basic Research (grants 18-04- 00243-a and 19-54-25001-Кипр_а). 


\section{REFERENCES}

Abdurakhmanov, G. M. \& Nabozhenko, M. V. (2011): Opredelitel' i katalog zhukov-chernotelok (Coleoptera: Tenebrionidae s. str.) Kavkaza i yuga evropeyskoy chasti Rossii [Keys and catalogue to darkling beetles (Coleoptera: Tenebrionidae s. str.) of the Caucasus and south of European part of Russia]. - KMK Scientifi c Press Ltd, Moscow, 361 pp. [in Russian, with an English summary]

Allard, E. (1882): Essai de classification des blapsides de 1'ancien monde. $4^{\mathrm{e}}$ et dernière partie. - Annales de la Société Entomologique de France (6) 2: 77-140.

Arnoldi, L. V. \& Medvedev G. S. (1969): Darkling beetles (Tenebrionidae). Pp. 398-408. In: Arnoldi, L. V. (ed): Biocomplex studies in Kazakhstan. T. 1. Plant communities and animal population of the steppes and deserts of Central Kazakhstan. - Nauka, Leningrad.

Ballion, E. E. (1878): Verzeichniss der im Kreise von Kuldsha gesammelten Käfer. - Bulletin de la Société Impériale des Naturalistes de Moscou 53 (1): 253-389.

Bogatchev, A. V. (1947): K systematike triby Blaptini (Tenebrionidae, Coleoptera) [To the taxonomy of the tribe Blaptini (Tenebrionidae, Coleoptera]. - Doklady Akademii Nauk Arerbaydzhanskoy SSR 3 (11): 513-515. [In Russian]

Castro Tovar, A. (2014): Una nueva especie de Blaps Fabricius, 1775 del sureste de España (Coleoptera, Tenebrionidae). - Arquivos Entomolóxicos 12: 237-243.

Chigray, I. A., Abdurakhmanov, G. M., Nabozhenko, M. V. \& Shapovalov, A. M. (2016): On Blaps Fabricius, 1775 (Coleoptera: Tenebrionidae) from Western Kazakhstan with the description of a new species from Tyuleniy Archipelago (Caspian Sea). - Zootaxa 41(1) 1-17. https://doi.org/10.11646/zootaxa.4173.1.1

Chigray, I. A. \& Nabozhenko, M. V. (2016): To the knowledge of the genus Blaps Fabricius, 1775 (Coleoptera: Tenebrionidae) from Iran and Transcaucasia. - Annales zoologici 66(2): 267-275. https://doi.org/10.3161/00034541ANZ2016.66.2.007

Condamine, F. L., Soldati, L., Rasplus, J.-Y. \& Kergoat, G. J. (2011): New insights on systematics and phylogenetics of Mediterranean Blaps species (Coleoptera: Tenebrionidae: Blaptini), assessed through morphology and dense taxon sampling. - Systematic Entomology 36: 340-361. https://doi.org/10.1111/j.1365-3113.2010.00567.x

Eversmann, E. F. von (1833): [new taxon]. In: Faldermann, F. (1833): Species novae Coleopterorum Mongoliae et Sibiriae. - Bulletin de la Société des Naturalistes de Moscou 6: $46-72$.

Faldermann, F. (1837): Fauna entomologica trans-caucasica. Pars II. Coleoptera trans-caucasica. - Nouveaux Mémoires de la Société Impériale des Naturalistes de Moscou 5: 1-433.

Fischer von Waldheim, G. (1820): Entomographie de la Russie [Entomographia Imperii Rossici]. Auctoritate Societatis Caesareae Mosquensis naturae scrutatorum collecta et in lucem edita. Volume I. - Augusti Semen typographi, Mosquae, 17 pls [Coleoptera] + 9 pls [other insects].

Fischer von WALDHeIm, G. (1822): Entomographie de la Russie [Entomographia Imperii Rossici]. Auctoritate Societatis Caesareae Mosquensis naturae scrutatorum collecta et in lucem edita. Volumen I. - Augusti Semen typographi, Mosquae, viii + 210 pp.

Fischer von Waldheim, G. (1832): [new taxa]. In: Ménétriés, E.: Catalogue raisonné des objets de zoologie recueillis dans un voyage au Caucase et jusqu'aux frontières actuelles de la Perse entrepris par ordre de S. M. l'Empereur. - Imprimerie Impériale des Sciences, St.Pétersbourg, xxxiii $+271+\mathrm{iv}+(1) \mathrm{pp}$.

Fischer von WALDHeim, G. (1844): Spicilegum Entomographiae Rossicae. II. Heteromera. Bulletin de la Société Impériale des Naturalistes de Moscou 17: 3-144. 
Gebler, F. A. (1825): Coleopterorum Sibiriae species novae. Pp. 42-57. In: Hummel, A. D. (ed.): Essais Entomologiques, Insectes de 1824. Novae species. Vol. 1, Nr. 4. - Chancellerie privée du Ministère de 1'Intérieur, St.-Pétersbourg, 71 pp.

Gebler, F. A. (1844): Charakteristik der von Hn. Dr. Schrenk in den Jahren 1842 und 1843 in den Steppen der Dsungarei gefundenen neuen Coleopterenarten. - Bulletin de la Classe Physico-Mathématique de l'Académie Impériale des Sciences de St.-Pétersbourg (2) 3 [1844-1845]: 97-106.

KAszAB, Z. (1963): Angaben zur Kenntnis der Tenebrioniden des Tschadsee-Gebietes, nebst einer Revision der afrikanischen Mesomorphus-Arten (Coleoptera). - Revue de Zoologie et de Botanique Africaines 68: 341-385.

Kaszab, Z. (1970): Beiträge zur Kenntnis der Fauna Afghanistans (Sammelergebniss von O. Jakeš 1963-64, D. Povolný \& Fr. Tenora 1966, J. Simek 1965-66, D, Povolný, J. Geiser, Z. Šebek \& Fr. Tenora 1967). Tenebrionidae, Col. - Časopis Moravského Musea, Vědy př́rodní 54 (Suppl.): 5-182 + 23 pls.

Kergoat, G. J., Soldati, L., Clamens, A.-L., Jourdan, H., Jabbour-Zahab, R., Genson, G., Bouchard, P. \& Condamine, F. L. (2014): Higher-level molecular phylogeny of darkling beetles (Coleoptera, Tenebrionidae). - Systematic Entomology 39(3): 486-499. https://doi.org/10.1111/syen.12065

Löbl, I., Nabozhenko, M. V. \& Merki, O. (2008): Tribe Blaptini Leach, 1815. Pp. 219-257. In: Löbl, I. \& Smetana, A. (eds): Catalogue of Palaearctic Coleoptera. Vol. 5. Tenebrionoidea. - Apollo Books, Stenstrup.

Marsham, T. (1802): Entomologia Britannica, sistens Insecta Britanniae indigena, secundum methodum Linnaeanam disposita. Tomus I. Coleoptera. - Wilks \& Tailor, London, 547 pp. https://doi.org/10.5962/bhl.title.65388

Martínez Fernández, J. C. (2010): Un nuevo representante del género Blaps Fabricius, 1775 de la Península Ibérica: Blaps tichyi n. sp. (Coleoptera, Tenebrionidae). - Boletín de la Sociedad Entomológica Aragonesa 47: 181-185.

Matthews, E. G. \& Bouchard, P. (2008): Tenebrionid beetles of Australia: Descriptions of tribes, keys to genera, catalogue of species. - Australian Biological Resources Study, Canberra, $410 \mathrm{pp}$.

Medvedev, G. S. (2001): Evolution and system of darkling beetles of the tribe Blaptini (Coleoptera, Tenebrionidae). - Chteniya pamyati N. A. Cholodkovskogo. Iss. 53. Russian Entomological Society Publ., Saint Petersburg, 332 pp. [in Russian]

Medvedev, G. S. (2004): New species of darkling beetles (Coleoptera, Tenebrionidae) from Palaearctic deserts. - Entomological Review 84(5): 558-580.

Medvedev, G. S. \& Iwan, D. (2006): Notes on the taxonomy of tenebrionid beetles (Coleoptera: Tenebrionidae). - Annales Zoologici 56(4): 613-621. https://doi. org/10.3161/000345406779508633

Medvedev, G. S. \& Nepesova, M. G. (1985): Opredelitel' Zhukov-chernotelok Turkmenistana [Key to darkling beetles of Turkmenistan]. - Ylym, Ashgabat, 180 pp. [in Russian]

MÉnÉTriés E. (1832): Catalogue raisonné des objets de zoologie recueillis dans un voyage au Caucase et jusqu'aux frontières actuelles de la Perse entrepris par l'ordre de S. M. I'Empereur. - Académie des Sciences, St. Petersburg, xxxiii + 272 + iv + (1) pp. https://doi.org/10.5962/ bhl.title.63878

MÉnÉTRIÉs, E. (1849): Catalogue des insectes recueillis par feu M. Lehmann avec les descrip tions des nouvelles espèces. (Seconde et dernière partie). Mémoires de l'Académie Impériale des Sciences de Saint-Pétersbourg, Sixième Série, Sciences Mathématiques, Physiques et Naturelles 8 [= Sciences Naturelles 6]: 217-238 + pls 3-4. 
Motschulsky, V. De (1845): Remarques sur la collection de coléoptères russes de Victor de Motschulsky. - Bulletin de la Société Impériale des Naturalistes de Moscou 18(1): 3-127.

Nabozhenko, M. V., Chigray, I. A., Poggi, R. \& Soldati, L. (2019): Little known darkling beetle Blaps scabiosa Baudi di Selve, 1874 (Coleoptera, Tenebrionidae: Blaptini): taxonomy, morphology, and distribution. - Entomological Review 99(7): 1035-1041. https://doi.org/10.1134/S0013873819070157

Ren, G. D., WANG, X. P. \& YU, Y. Z. (2000): Nine records and one new species of the tribe Blaptini from China (Coleoptera: Tenebrionidae). - Journal of Hebei University (Natural Science Edition) 20 (Suppl.): 26-33.

Ren, G. D., BA, Y., Liu, H., Niu, Y., Zhu, X., LI, Z. \& ShI, A. (2016): Fauna Sinica: Insecta. Volume 63: Coleoptera: Tenebrionidae (I). - Science Press, Beijing, 532 pp. [in Chinese with an English summary]

Schawaller, W. (2006): The genus Blaps Fabricius, 1775 in the Nepal Himalayas (Coleoptera, Tenebrionidae). - Entomologica Basiliensia et Collectionis Frey 28: 193-203.

Seidlitz, G. (1893): Tenebrionidae. Pp. 201-400. In: Kiesenwetter, H. von \& Seidlitz, G. von (eds): Naturgeschichte der Insecten Deutschlands begonnen von Dr. W.F. Erichson, fortgesetzt von Prof. Dr. H. Schaum, Dr. G. Kraatz, H. v. Kiesenwetter, Julius Weise, Edm. Reitter und Dr. G. Seidlitz. Erste Abtheilung. Coleoptera. Fünfter Band. Erste Hälfte. Nicolaische Verlags-Buchhandlung, Berlin.

Semenov Tian-Shansky, A. P. \& Bogatchev, A. V. (1936): Supplément à la révision du genre Blaps F. (Coleoptera, Tenebrionidae) de G. Seidlitz, 1893. - Festschrift zum 60 Geburtstage von Professor Dr. Embrik Strand 1: 553-568.

Sкорім, N. G. (1960): Material on the morphology and the ecology of larvae of the genus Blaptini (Coleoptera, Tenebrionidae). - Trudy Instituta zooologii Akademii nauk Kazakhskoy SSR 11: 36-71. [in Russian]

SкоріN, N. G. (1961): Materials on the fauna and the ecology of darkling beetles (Coleoptera, Tenebrionidae) of southeastern Kazakhstan. - Trudy Nauchno-Isledovatelskogo Instituta Zashchtity Rasteniy Kazakhstanskoy Akademii Selskokhozyastvennykh Nauk 6: 172-208. [in Russian]

Sкоріл, N. G. (1964): Neue Tenebrioniden aus Zentralasien III, nebst einigen systematischen sowie synonymischen Bemerkungen. - Annales historico-naturales Musei nationalis hungarici 56: 389-412.

Sкоріл, N. G. (1966): Neue Tenebrioniden aus Zentralasien IV, nebst einigen systematischen sowie synonymischen Bemerkungen. - Annales historico-naturales Musei nationalis hungarici 58: 325-342.

SкоріN, N. G. (1968): Darkling beetles (Coleoptera, Tenebrionidae) of South Kazakhstan and their economic significance. - Trudy Kazakhskogo nauchno-issledovatel'skogo instituta zashchity rasteniy 10: 74-114. [in Russian]

Sкоріл, N. G. (1973): Darkling beetles (Coleoptera, Tenebrionidae) from Ballion's collection collected in Kuldzha and adjacent regions of Kazakhstan and Kirgizia. - Entomological Review 52(4): 555-561.

Sкоріл, N. G. (1977): Über Blaps transversimsulcata Ball. und ihrer näheren Verwandten (Coleoptera, Tenebrionidae). - Annales historico-naturales Musei nationalis hungarici 69: 149-151.

Sкоріл, N. G. \& Kаszaв, Z. (1978): Über die Arten der Gattung Blaps F. (Coleoptera, Tenebrionidae), gesammelt von Herrn Dr. W. Wittmer im Jahre 1976 in Kashmir. - Folia entomologica hungarica 31: 207-212. 
Soldati, L., Kergoat, G. J. \& Condamine, F. (2009): Important notes on taxonomic structure of Blaps nitens Laporte de Castelnau, 1840 with the description of new subspecies Blaps nitens medvedevi subsp. n. (Coleoptera: Tenebrionidae: Blaptini). - Caucasian Entomological Bulletin 5(2): 231-233. https://doi.org/10.23885/1814-3326-2009-5-2-231-233

Soldati, L., Condamine, F. L., Clamens, A.-L. \& Kergoat, G. J. (2017): Documenting tenebrionid diversity: progress on Blaps Fabricius (Coleoptera, Tenebrionidae, Tenebrioninae, Blaptini) systematics, with the description of five new species. - European Journal of Taxonomy 282: 1-29. https://doi.org/10.5852/ejt.2017.282

Zоuвкоғғ, B. (1829): Sur un nouveau genre et quelques nouvelles espèces de coléoptères. Bulletin de la Societě Impériale des Naturalistes de Moscou 1: 147-170.

Zoubкоғғ, B. (1833): Nouveaux coléoptères recueillis en Turcménie. - Bulletin de la Société Impériale des Naturalistes de Moscou 6: 310-340.

Received December 5, 2019, accepted April 15, 2020, published May 15, 2020 